Ortuste Quiroga et al.

\title{
1 Mechanosensitive Ion Channel Piezo1 Regulates Myocyte Fusion during
}

\section{Skeletal Myogenesis}

5 Huascar Pedro Ortuste Quiroga ${ }^{1}$, Shingo Yokoyama $^{2}$, Massimo Ganassi $^{3}$, Kodai Nakamura ${ }^{1}$,

6 Tomohiro Yamashita ${ }^{1}$, Daniel Raimbach ${ }^{4}$, Arisa Hagiwara ${ }^{1}$, Atsushi Asakura ${ }^{5}$, Yoshiro

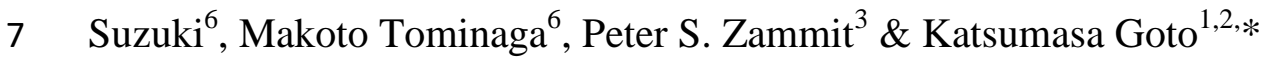

$9 \quad{ }^{1}$ Department of Physiology, Graduate School of Health Sciences, Toyohashi SOZO

10 University

11 22aboratory of Physiology, School of Health Sciences, Toyohashi SOZO University

$12{ }^{3}$ Randall Centre for Cell and Molecular Biophysics, King's College London, London, SE1

13 1UL, UK

$14{ }^{4}$ Centre of Human and Aerospace Physiological Sciences, King's College London, London, 15 SE1 1UL, UK

${ }^{5}$ Stem Cell Institute, Paul \& Sheila Wellstone Muscular Dystrophy Center, Department of

17 Neurology, University of Minnesota Medical School

${ }^{6}$ Division of Cell Signalling, National Institute for Physiological Sciences

* Address for correspondence;

Katsumasa Goto, Ph.D. 
bioRxiv preprint doi: https://doi.org/10.1101/2020.09.27.315242; this version posted November 22, 2020. The copyright holder for this

preprint (which was not certified by peer review) is the author/funder, who has granted bioRxiv a license to display the preprint in perpetuity. It is made available under aCC-BY-NC-ND 4.0 International license.

Ortuste Quiroga et al.

TEL +81-50-2017-2272

25

FAX $+81-532-55-0803$

26

E-mail: gotok@ sepia.ocn.ne.jp

27

28 


\section{Abstract}

30 Mechanical stimuli such as stretch and resistance training are essential to regulate growth and function of

31 skeletal muscle. However, the molecular mechanisms involved in sensing mechanical stress remain unclear. Here, the purpose of this study was to investigate the role of the mechanosensitive ion channel Piezo1 during myogenic progression. Muscle satellite cell-derived myoblasts and myotubes were modified with stretch, siRNA knockdown and agonist-induced activation of Piezo1. Direct manipulation of Piezo1 modulates terminal myogenic progression. Piezol knockdown suppressed myoblast fusion during myotube formation and maturation. This was accompanied by downregulation of the fusogenic protein Myomaker. Piezol knockdown also lowered $\mathrm{Ca}^{2+}$ influx in response to stretch. Conversely Piezo1 activation stimulated fusion and increased $\mathrm{Ca}^{2+}$ influx in response to stretch. These evidences indicate that Piezo1 is essential for myotube formation and maturation, which may have implications for msucular dystrophy prevention through its role as a mechanosensitive $\mathrm{Ca}^{2+}$ channel. 
Ortuste Quiroga et al.

\section{Introduction}

Skeletal muscle is a highly specialised tissue composed of multi-nucleated, postmitotic muscle fibres. Since myonuclei within a muscle fibre do not divide after development, the production of new myonuclei is entrusted to satellite cells (SCs), the skeletal muscle's resident stem cell. SCs are found on the surface/periphery of postnatal skeletal muscle fibres [1-3] and in response to muscle damage, rapidly activate to generate a myoblast progeny that proliferate, undergo myogenic differentiation, and fuse to repair damaged myofibres, resulting in regeneration of a functional muscle [4]. Although much is known about the myogenic progression program, some of the underlying mechanisms remain to be determined. Among these are the molecular mechanisms involved in sensing mechanical stress (mechanosensation) and its effect on myocyte fusion at the cellular and molecular levels.

Mechanosensitive (MS) ion channels are pore-forming membrane proteins which gate in response to mechanical stimuli applied on the cell membrane [5-7]. MS ion channels have been linked to many physiological processes associated with mechanosensory transduction; including osmoregulation, proprioception, hearing, touch, blood flow regulation to name but a few examples [8-10]. Piezo1 and Piezo2 were first identified by Coste et al, (2010) as the long-sought principal types of molecular force sensors (mechanosensors) in mammalian cells [5]. Piezo1 (and Piezo2) is a very large protein containing 2500 amino acids with each subunit (a total of three subunits per channel) containing an estimated 24-40 transmembrane (TM) segments $[6,8,11,12]$. Characterisation of ionic selectivity revealed that Piezo1 was nonselective, permeating $\mathrm{Na}^{+}, \mathrm{K}^{+}, \mathrm{Ca}^{2+}$ and $\mathrm{Mg}^{2+}$ with a preference for $\mathrm{Ca}^{2+}[5,13]$. With such a crucial role $\mathrm{Ca}^{2+}$ regulation plays in skeletal muscle maintenance and repair, understanding Piezol's function may prove vital when looking at strategies for therapeutic interventions of muscular dystrophies [14,15]. 
Ortuste Quiroga et al.

Piezo1 is widely expressed in a range of tissues, including skeletal muscle, the bladder, colon, lung, skin and stomach $[5,16]$. Piezo2 shows a similar pattern with the exception of being highly expressed in the dorsal root ganglion (DRG) while being less represented in skeletal muscle $[5,16]$. Another key difference between Piezo1 and Piezo2 is their activation and inactivation kinetics. Overexpression analyses revealed that the kinetics of inactivation of Piezo2-dependent mechano-activated currents was faster than Piezo1 both for inward and outward currents, thus conferring distinct channel properties [5,13,11]. Although Tsuchiya et al published the first report on Piezo1 in skeletal muscle cells [17], looking at the role of transmembrane localisation of phosphatidylserine and Piezo1 activation, the physiological function(s) of Piezo1 throughout the myogenic program remain unelucidated.

The current study aimed to analyse the role of Piezo1 in skeletal muscle proliferation, differentiation and its role in stretch-induced $\mathrm{Ca}^{2+}$ influx of primary derived myotubes. Our findings revealed that Piezo1 is dispensable for myoblast proliferation and onset of differentiation, but its knockdown suppressed myotube formation and maturation in primary myotubes derived from slow soleus and fast extensor digitorum longus (EDL) muscles in mice. In line, Piezol reduction was accompanied by downregulation of the fusogenic gene Myomaker, decreased accumulation of f-actin and lowered $\mathrm{Ca}^{2+}$ influx of myotubes in response to mechanical stretch. In contrast, administration of the Piezo1-specific agonist Yoda1 increased fusion of myoblasts. Piezo1 activation also showed increased $\mathrm{Ca}^{2+}$ influx in response to stretch. Using publicly available datasets, we showed a dysregulation in the expression of Piezo1 in the skeletal muscle disease facioscapulohumeral muscular dystrophy (FSHD). In summary, we propose that Piezo1 plays a crucial role at the terminal stage of myotube fusion and maturation. Piezo1 may employ direct or indirect mechanisms that regulate fusion proteins as well as proteins involved in cytoskeletal organisation. These 
Ortuste Quiroga et al.

mechanisms may be regulated by intracellular $\mathrm{Ca}^{2+}$-signals mediated by mechanosensitive Piezo1 channels.

\section{Materials and methods}

\section{Primary myoblast cell culture}

All experimental procedures were carried out in accordance with the Guide for the Care and Use of Laboratory Animals as adopted and promulgated by the National Institutes of Health (Bethesda, MD, USA) and were approved by the Animal Use Committee of Toyohashi SOZO University A2018006, A2019006). Male C57BL/6J mice (8-12 weeks of age) were used. All mice were housed in a vivarium room with 12-h-12-h light-dark cycle; with temperature and humidity maintained at $\sim 23^{\circ} \mathrm{C}$ and $\sim 50 \%$, respectively. Solid food and water were provided ad libitum.

After cervical dislocation, the EDL (extensor digitorum longus) and soleus muscles were carefully dissected, and manipulated only by their tendons. Muscles were digested in 0.2\% Collagenase Type 1 (Sigma, UK. Ref: SCR103) in Dulbecco's Modified Eagle Medium (DMEM, Gibco, Thermo Fisher Scientific, Ref: 11885084) with 1\% penicillin/streptomycin (Pen Strep, Gibco, Thermo Fisher Scientific, Ref: 15140-122) for 2 hours. Individual myofibres were then dissociated by trituration using heat polished glass Pasteur pipettes (Marienfeld, Germany. Ref: 3233049) with variously sized apertures (coated with 5\% BSA, Sigma-Aldrich, Ref: A7906-100G) and washed as described by Collins and Zammit (2009)[18]. Isolated myofibres were plated on Matrigel (Corning. Ref: 354234) and the satellite cell-derived myoblasts were then expanded in proliferation medium, consisting of; DMEM, with $30 \%$ heat-inactivated foetal bovine serum (FBS) (Gibco, Thermo Fisher Scientific. Ref: 26140-079), 10\% horse serum (Gibco, Thermo Fisher Scientific, Ref: 16050- 
Ortuste Quiroga et al.

122), $1 \%$ chick embryo extract (Sera Laboratories. Ref: CE-650-TL), $10 \mathrm{ng} / \mathrm{ml}$ basic FGF (bFGF, Gibco, Thermo Fisher Scientific. Ref: PHG0264) and 1\% penicillin. Cells were kept in a $37^{\circ} \mathrm{C}$ incubator (Panasonic, MCO-230AICUVH) under a humidified atmosphere with 95\% air and $5 \% \mathrm{CO}_{2}$. Cells designated for proliferation and differentiation conditions were seeded at different densities depending on the size of wells they were cultured in. For 96-well plate proliferation conditions required 5,000 cells per well, and for differentiation, cells were seeded at 10,000 cells per well. For 6-well plates, proliferating and differentiation cohorts consisted of 50,000 cells and 70-80,000 per well, respectively. Differentiation medium was made up of DMEM, $2 \%$ heat-inactivated horse serum, and $1 \%$ penicillin.

\section{siRNA transfection}

Small interfering RNAs (siRNAs) were purchased from (Qiagen, Hiden, Germany) (Table 1) and diluted to 20 or $10 \mu \mathrm{M}$ in double-distilled water (ddH2O) and stored at $-20^{\circ} \mathrm{C}$. To investigate the effects Piezol knockdown on proliferation, early entry into differentiation and myotube formation, Piezol-targeting or control scrambled siRNA (siScrambled; Qiagen, Hiden, Germany) was transfected in proliferation medium. Cells were plated on 6-well plates at 50,000 cells per well in proliferation medium. Following a $24 \mathrm{~h}$ incubation period, the medium was replaced with $1.75 \mathrm{ml}$ fresh proliferation medium and the transfection mixture was prepared: A solution $150 \mu$ l Optimem (Gibco, Thermo Fisher Scientific. Ref: 31985-070) medium with $9 \mu \mathrm{l}$ of lipofectamine (lipofectamine RNAiMAX Thermo Fisher Scientific. Ref: 13778030) was made for each well. Separately, siRNA was diluted in $150 \mu$ l Optimem. The two solutions were then mixed and incubated for $5 \mathrm{~min} .250 \mu \mathrm{l}$ of the siRNA/lipofectamine mixture was added to corresponding wells dropwise. The final siRNA concentration was set at $10 \mathrm{nM}$. Following overnight incubation in the transfection medium, cells were trypsinised for RT-qPCR analysis and seeded in 96-well plates for proliferation and differentiation 
Ortuste Quiroga et al.

cohorts (day 1 and day 3 differentiation) in proliferation and differentiation medium, respectively. After a 24-hour incubation, proliferating cells were subjected to a 2-hour 5cohorts were also fixed. in early differentiated myotubes. Cells were seeded at confluency in differentiation medium. Following 24-hour incubation in differentiation medium, siRNA transfection was performed.

Table 1. List of siRNAs used

\begin{tabular}{|l|l|l|}
\hline \multicolumn{1}{|c|}{ Gene } & \multicolumn{1}{|c|}{ Species } & \multicolumn{1}{c|}{ siRNA ID } \\
\hline $\begin{array}{l}\text { scrambled non-targeting siRNA (All } \\
\text { Stars Negative Control siRNA) }\end{array}$ & Mouse & Qiagen, 1027281 \\
\hline Piezo1 & Mouse & Qiagen, S104420409 \\
\hline Piezol & Mouse & Qiagen, S104420402 \\
\hline Piezol & Mouse & Qiagen, S100814807 \\
\hline Piezol & Mouse & Qiagen, S100814821 \\
\hline
\end{tabular}


Ortuste Quiroga et al.

\section{RNA Extraction and Reverse Transcription}

RNA was extracted from cells using the RNeasy mini kit as per manufacturer's requirements (Qiagen. Ref: 217004). Reverse transcription was carried out using PrimeScript RT Master Mix (Takara Bio, Otsu, Japan. Ref: RR036A). Optical density analysis using a Nanodrop ND-1000 spectrophotometer (Labtech, UK) quantified RNA concentration. Samples were then loaded to a PCR thermal cycler (Takara, Dicemini). The resulting cDNA was then diluted 1:9 to obtain a working dilution for RT-qPCR analysis.

\section{Real-time Quantitative PCR (RT-qPCR)}

Primers were designed using the Takara Bio Perfect Real Time Support System (Takara Bio, Table 2). Primers were diluted to $50 \mu \mathrm{M}$ in $\mathrm{ddH}_{2} \mathrm{O}$ and stored at $-20^{\circ} \mathrm{C}$. Realtime RT-qPCR was performed on the cDNA (Thermal Cycler Dice Real Time System IIMRQ, Takara Bio) using Takara SYBR Premix Ex Taq II (Takara Bio. Ref: RR802A). 12.5 $\mu 1$ of SYBR Premix Ex were added to each RT-qPCR well. $8.5 \mu 1$ of ddH20 and $2 \mu 1$ of the corresponding primers were then added (a final concentration of $2 \mu \mathrm{M}$ per primer). $2 \mu \mathrm{l}$ of the respective cDNA was then added to the appropriate wells, bringing the total volume to $25 \mu 1$ per well. The RT-qPCR cycle consisted of $95^{\circ} \mathrm{C}$ for $30 \mathrm{~s}$ (for enzyme activation), followed by 40 cycles at $95^{\circ} \mathrm{C}$ for $5 \mathrm{~s}$ and a qPCR amplification period of $30 \mathrm{~s}$ at $60^{\circ} \mathrm{C}$. The relative fold change of expression was calculated by the comparative threshold cycle (CT) method using Takara Thermal Cycler Dice Real Time System Software Ver. 4.00 (Takara Bio). To normalise for the amount of total RNA present in each reaction, Gapdh was used as an internal standard. 
Ortuste Quiroga et al.

Table 2. Real Time quantitative PCR (RT-qPCR) primers.

\begin{tabular}{|c|c|c|c|c|}
\hline Gene & Species & Forward primer (5'-3') & Reverse primer (5'-3') & Reference \\
\hline Gapdh & Mouse & TGTGTCCGTGGATCTGA & TTGCTGTTGAAGTCGCAGGAG & $\begin{array}{c}\text { Takara } \\
\text { Bio, } \\
\text { MA050371 }\end{array}$ \\
\hline Piezo1 & Mouse & CTTTATCATGAAGTGCAGCCGAG & CCAGATGATGGCGATGAGGA & $\begin{array}{c}\text { Takara } \\
\text { Bio, } \\
\text { MA125411 }\end{array}$ \\
\hline Myomaker & Mouse & CATGCGCCGTGACATTCTG & AAGCATTGTGAAGGTCGATCTCTG & $\begin{array}{c}\text { Takara } \\
\text { Bio, } \\
\text { MA131293 }\end{array}$ \\
\hline Myomixer & Mouse & GAATCCACCGCAGGCAAA & ACCATCGGGAGCAATGGAAC & $\begin{array}{c}\text { Takara } \\
\text { Bio, } \\
\text { MA101853 }\end{array}$ \\
\hline
\end{tabular}

187

\section{Piezol activation}

In order to induce Piezol activation, early forming myotubes were subjected to the

Piezo1 specific agonist Yoda1 (Cayman Chemical Company. Ref: 21904) diluted in dimethyl

period in differentiation medium at high confluency (10,000 cells/well). By this point primary derived myoblasts seeded at high confluency, begin to show myotube formation in the

Yoda1 binds the agonist transduction motif (ATM), located at the pore domain of the Piezo1 channel [19]. With each subunit displaying such motif, Yoda1 has potentially three binding sites. This phase of the investigation consisted of two main variables 1) drug concentration and 2) duration of time cells were incubated with the drug. Five concentrations were chosen in order to cover an increasing spectrum of Yoda1 final concentration, these were: $5,10,30$ and $100 \mu \mathrm{M}$ diluted in differentiation medium. Preliminary findings from the group found that a 24-hour incubation with any of the concentrations chosen, led to complete abolishment 
Ortuste Quiroga et al.

202

203

204

205

206

207

208

209

210

211

212

213

214

215

216

217

218

219

220

221

222

of myotube maturation (data not show). Thus, time-points thought to have potential to maximise myotube fusion/maturation were tested. The incubation time-points were set for 1 and 30 min, 1 and 4 hours. Control cohorts containing only DMSO were incubated at the allocated times to allow comparisons to be made within each condition. Following the incubation of Yoda1 or DMSO, cells were cultured in the differentiation medium for a further 2 days (i.e. myotubes were analysed 3 days post initial induction of differentiation).

\section{Immunolabelling}

Throughout the protocol, all washes were performed with Dulbecco's phosphatebuffered saline (DPBS, Gibco, Dulbecco's Phosphate Buffered Saline, Thermo Fisher Scientific, Ref: 14190-144). Cells were fixed with 4\% paraformaldehyde for $15 \mathrm{~min}$. Samples were then washed three times with PBS (5 minutes each wash) and permeabilised for 15 min using 0.5\% triton-X100/PBS (Sigma-Aldrich. Ref: T9284-500ml). Cells were blocked for 1 hour in 5\% bovine serum albumin (BSA, Sigma-Aldrich Ref: A7906-100G). Primary antibodies (diluted to the working concentration in PBS) (Table 3) were added to the samples and incubated overnight at $4^{\circ} \mathrm{C}$. Primary antibodies were decanted, the samples were washed three times and appropriate secondary antibodies diluted to the working concentration in PBS added (Table 3) were added to the samples. The Samples were covered with aluminium foil to avoid light exposure and left to stand at room temperature for 1 hour. Cells were washed again (three times). To visualise nuclei, the cells were incubated for $10 \mathrm{~min}$ at room temperature with $1 \mu \mathrm{g} / \mathrm{ml} \mathrm{4}$, 6-diamidino-2-phenylindole (DAPI) (Sigma. Ref: D954210MG) diluted 1:1000 in PBS. After a final wash with PBS (5 min), cells were replenished with PBS and stored at $4{ }^{\circ} \mathrm{C}$ until image analysis. 
Ortuste Quiroga et al.

Table 3. Primary and Secondary antibodies used

\begin{tabular}{|c|c|c|}
\hline Primary antibody & Dilution & Reference \\
\hline Monoclonal mouse - myogenin & $1: 10$ & $\begin{array}{l}\text { Development Studies Hybridoma } \\
\text { Bank (DSHB), F5D-s }\end{array}$ \\
\hline $\begin{array}{l}\text { Monoclonal mouse }- \text { MF20 } \\
\text { (Myosin Heavy Chain) }\end{array}$ & $1: 10$ & $\begin{array}{l}\text { Development Studies Hybridoma } \\
\text { Bank (DSHB), MF20-s }\end{array}$ \\
\hline Secondary antibody & Dilution & Reference \\
\hline $\begin{array}{l}\text { Donkey anti-mouse } \operatorname{IgG}(\mathrm{H}+\mathrm{L}) \text {, } \\
\text { Alexa Fluor }{ }^{\circledR} 555\end{array}$ & $1: 500$ & Life Technologies, A21203 \\
\hline
\end{tabular}

\section{EdU incorporation}

For the evaluation of cell proliferation, cells were incubated with 5-ehtynyl-2'deoxyuridine (EdU: Invitrogen, Thermo Fisher Scientific) at $10 \mu \mathrm{M}$, added in fresh proliferation medium for 2 hours at $37{ }^{\circ} \mathrm{C}$. EdU, the alkyne-containing thymidine analog, is incorporated into DNA during active DNA synthesis. The click-iT EdU Alexa Flour kit (Invitrogen, Thermo Fisher Scientific, Click-iT, EdU Alexa Fluor. Ref: 594 C10339) was used as per manufacturer's instructions with either the 488 (green) or 594 (red) azide to detect incorporated EdU.

\section{Phalloidin labelling}

To evaluate the cytoskeleton, cells were treated with phalloidin (Invitrogen, Thermo Fisher Scientific, Alexa-Fluor. Ref: 488 A12379) diluted 1:40 in PBS. Phalloidin binds to factin, a major cytoskeleton protein in skeletal muscle fibres. Cells were incubated with 
Ortuste Quiroga et al.

phalloidin solution for 30 mins at room temperature. Cells were then washed with PBS twice for $5 \mathrm{~min}$.

\section{Image quantification}

Images were taken using a fluorescence microscope (BZ-X710, KEYENCE, Osaka,

Japan). Four to five images per each well (3 wells per repeat) consistent of a total of 12-15 images per repeat were analysed. One repeat refers to one mouse. For EdU incorporation, the

subsequently expressed as percentages. The relative proportion of cells expressing myogenin was also quantified in this manner.

The fusion index was calculated by quantifying the total number of nuclei within

positive myotube to be quantified for the fusion index: (MyHC-positive myotubes containing $\geq 2$ nuclei/total number of nuclei) $\times 100$.

To measure myotube width, the "measure" tool on ImageJ imaging software was used.

This allows measurements of a chosen distance to be made. Before measurement, a scale was applied to all images. On the "set scale" option pixels are converted into $\mu \mathrm{m}$. Taking the fluorescence microscope and magnification into account the program determines $100 \mu \mathrm{m}$ to be 133.00 pixels or $0.75 \mu \mathrm{m} /$ pixel. Three independent images were chosen per condition. The criterion for this analysis was to choose the widest possible distance between myotube edges points within the field of view. The values were then averaged. 
Ortuste Quiroga et al.

\section{Stretch experiments and intracellular $\mathrm{Ca}^{2+}$ level imaging}

silicone chambers (Strexcell, Ooyodonaka, Ref: STB-CH-0.02). After 24-hour incubation, cells were transfected with either control (siScrambled) or Piezol-specific siRNA in proliferation medium. After overnight incubation, cells were switched to differentiation medium and cultured for a further 3 days. Stretch experiments were conducted on the third day. For Piezo1 activation by Yoda1 administration, cells were seeded as above. Following the initial overnight incubation, cells were switched to differentiate and the resulting myotubes were analysed 3 days post differentiation induction. an extension device (modified version of STB-150, Strex) on the microscope stage. Stretch stimulation was applied using a pre-set stretch speed and distance. After an initial 1 min rest period $(0 \%$ stretch), stretch was applied at $3 \%(0.3 \mathrm{~mm}), 6 \%(0.6 \mathrm{~mm})$ and $9 \%(0.9 \mathrm{~mm})$ for

Yoda1 cohorts were administered with $30 \mu \mathrm{M}$ of the agonist before being subjected to stretch. Ionomycin (Sigma-Aldrich) at $5 \mu \mathrm{M}$ was applied at the final step in each experiment for normalisation and to check cell viability. Fura 2-AM at 340 and $380 \mathrm{~nm}$, and the emitted light signal was read at $510 \mathrm{~nm}$. Images were then analysed on ImageJ imaging software. Three independent myotubes from each condition 
Ortuste Quiroga et al.

were selected and analysed. The changes in ratios were calculated by subtracting basal values from peak values. The values were then normalised to ionomycin data.

\section{Statistical analysis}

Data is presented as mean \pm SEM from at least three experiments (at least three mice). Significance was assessed by either paired Student's t-test or one-way ANOVA followed by followed by the Tukey-Kramer post-hoc; wherein p-values of $<0.05$ were considered to be statistically significant. A paired t-test was adopted when comparing effects within the same group e.g. analysing the effects of siRNA mediated down-regulation of Piezol versus siRNA controls in murine derived myoblasts. A one-way ANOVA was implemented when two or more independent groups were analysed, for example; comparing the effects of varying agonist concentrations across different timepoints. 
Ortuste Quiroga et al.

\section{Results}

\section{Expression of Piezo1 in primary myoblasts and myotubes}

We investigated the expression level of Piezol during myogenic progression in murine fast EDL and slow soleus muscles-derived primary myoblasts (Figure 1a). The expression of EDL-derived cells (Figure 1b) showed a significant increase in mRNA expression of Piezol in myotubes cultured at 3 days in differentiation medium, compared to the expression level in proliferating myoblasts $(\mathrm{p}<0.05)$. Soleus-derived cells (Figure 1c) showed a significant increase in Piezol mRNA expression after 24 and 72 hours in differentiation medium, compared to proliferation cohorts $(\mathrm{p}<0.05)$. The observed increase in Piezol in soleusderived cells after 1 day of differentiation may be explained by the fact that soleus-derived myoblasts tend to differentiate slightly quicker than EDL, i.e. myotubes could be seen sooner in soleus-derived cells compared to EDL. Moreover, we found that Piezol is expressed at a higher level in day 1 and day 3 differentiated soleus cells compared to EDL counterparts (Figure S1).

\section{siRNA-mediated downregulation of Piezo1}

We next set out to evaluate the effects of manipulating Piezol on proliferation and myogenic differentiation. This was achieved by siRNA-mediated knockdown (Figure 1d and e). Cells were transfected with either control (siScrambled) siRNA (control-siRNA) or targeting siRNA specific for Piezol (Piezol-siRNA). Following a 24-hours incubation period, the medium was changed and cells were incubated for a further 24 hours. In EDL-derived cells (Figure 1d), the treatment of Piezol-siRNA showed a significant reduction $(\sim 48 \%)$ in mRNA expression level of Piezol, compared to control-siRNA ( $\mathrm{p}<0.05)$. Analysis of soleus- 
bioRxiv preprint doi: https://doi.org/10.1101/2020.09.27.315242; this version posted November 22, 2020. The copyright holder for this preprint (which was not certified by peer review) is the author/funder, who has granted bioRxiv a license to display the preprint in perpetuity. It is made available under aCC-BY-NC-ND 4.0 International license.

Ortuste Quiroga et al.

derived cells (Figure 1e) also revealed a significant reduction ( $34 \%)$ in expression of Piezol

326

post siRNA-mediated transfection, compared to control conditions $(\mathrm{p}<0.05)$. 
bioRxiv preprint doi: https://doi.org/10.1101/2020.09.27.315242; this version posted November 22, 2020. The copyright holder for this preprint (which was not certified by peer review) is the author/funder, who has granted bioRxiv a license to display the preprint in perpetuity. It is made available under aCC-BY-NC-ND 4.0 International license.

Ortuste Quiroga et al.
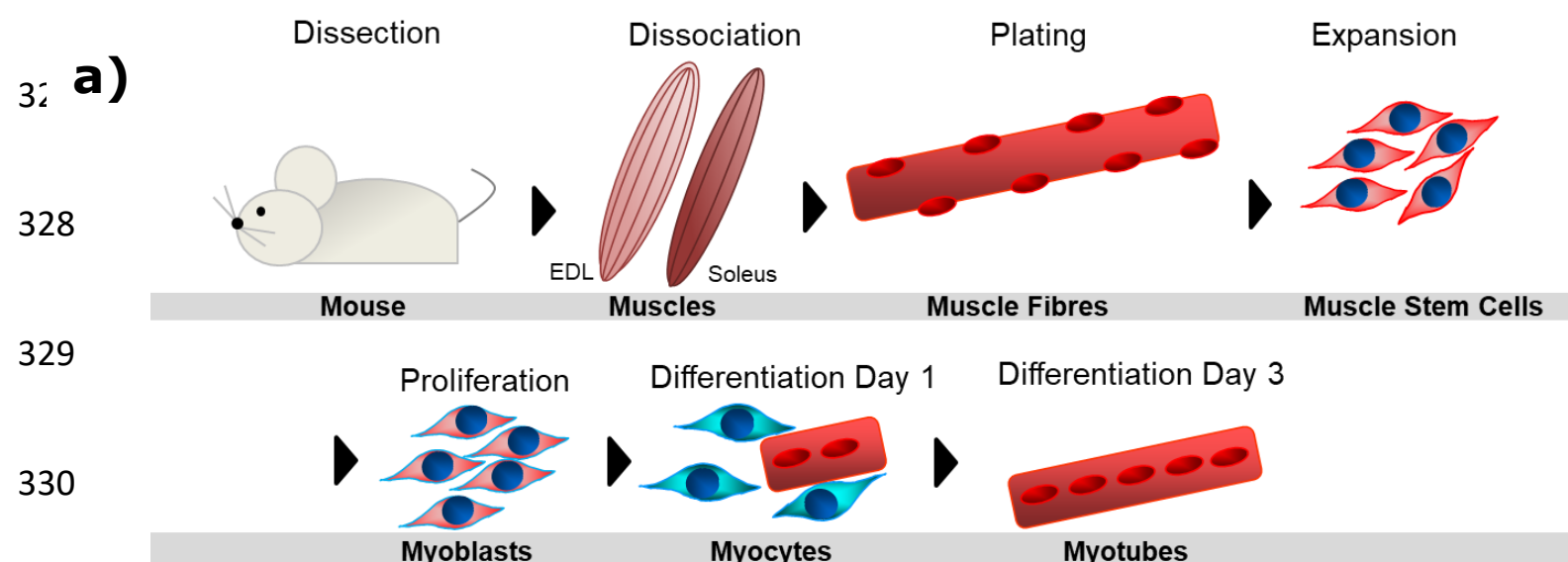

Differentiation Day 3
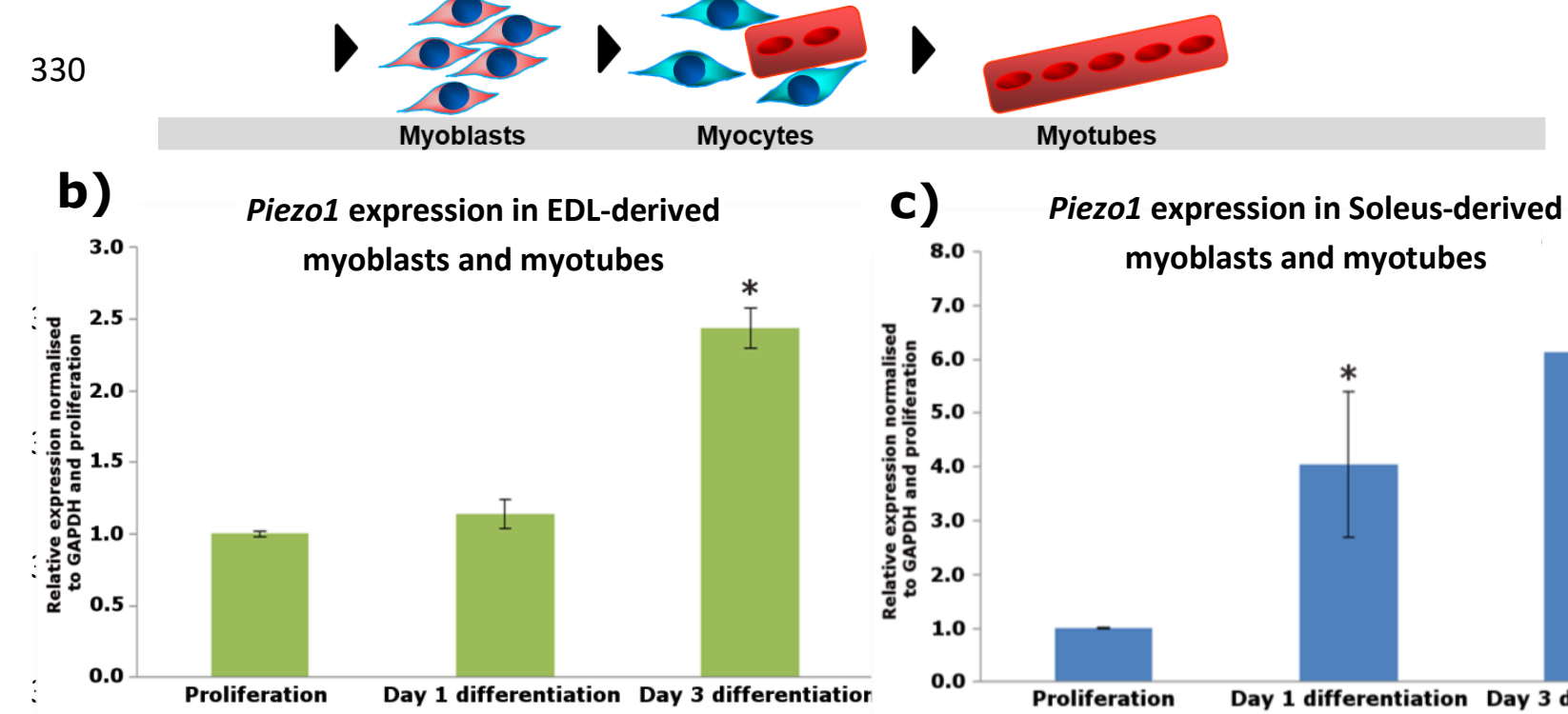

d)

$33 \iota$

f)

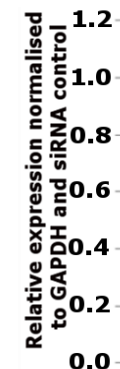

Piezo1 expression in EDL-derived myoblasts

\section{h)}

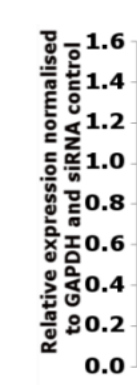

Piezo2 expression in EDL-derived myotubes

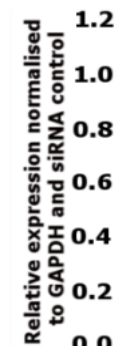

SiRNA control myotubes

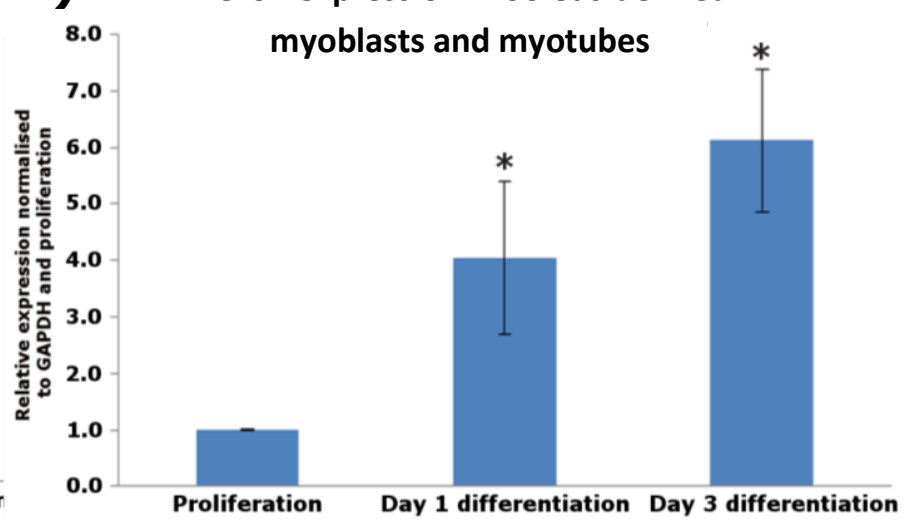

e)

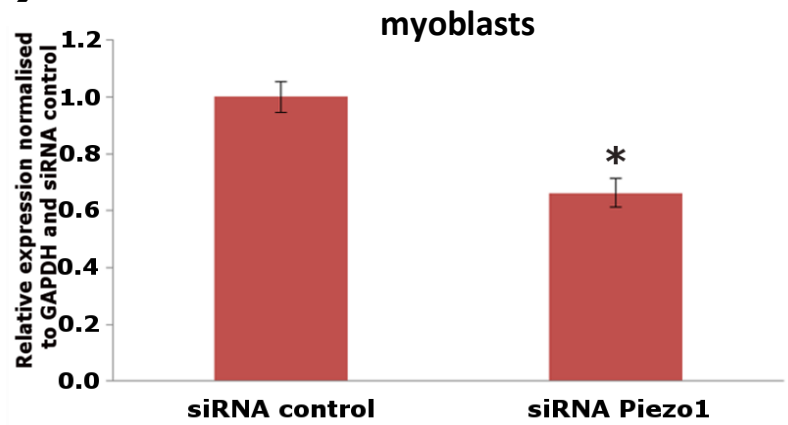

g)

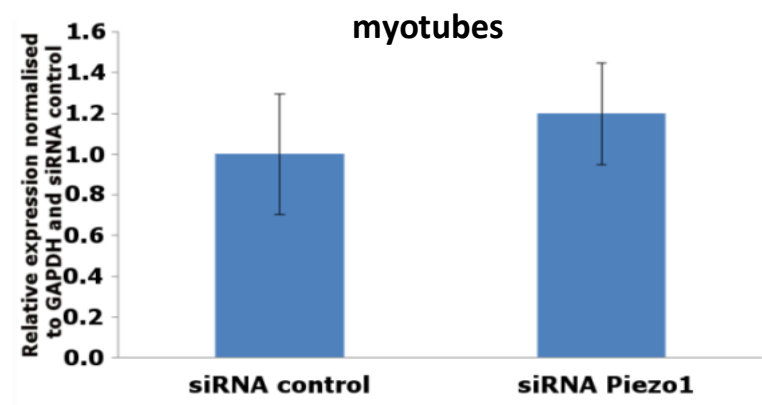

i)

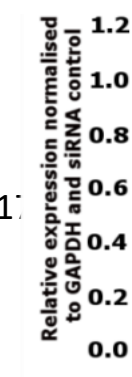

Piezo1 expression in Soleus-derived myotubes 
bioRxiv preprint doi: https://doi org/10.1101/2020.09.27.315242; this version posted November 22, 2020. The copyright holder for this preprint (which was not certified by peer review) is the author/funder, who has granted bioRxiv a license to display the preprint in perpetuity. It is made available under aCC-BY-NC-ND 4.0 International license.

Ortuste Quiroga et al.

345 Figure 1. Piezo1 is expressed in satellite cell-derived myoblasts throughout myogenic differentiation. Piezo1

346 downregulation does not alter the expression of Piezo2.

347 a) Schematic representation of muscle isolation and satellite cell expansion procedure. b) and c) Relative fold changes in 348 expression of Piezol in murine EDL and soleus muscle-derived myoblasts, during proliferation and through differentiation; 34924 hours (Day 1) and 72 hours (Day 3) in differentiation medium. d) and e) EDL and soleus-derived myoblasts were 350 transfected with $10 \mathrm{nM}$ of either control-siRNA (siScrambled) or targeting siRNA against Piezol (Piezol-siRNA. After 351 overnight incubation, cells were incubated for a further 24 hours and expression of Piezol was measured. f-i) Relative fold 352 changes in expression of Piezol and Piezo2 in EDL and soleus muscle-derived myotubes. Following an initial differentiation 353 period (24 hours at high confluency), cells were transfected with $10 \mathrm{nM}$ of either control-siRNA (siScrambled) or Piezol354 siRNA. After overnight incubation, cells were incubated for a further 24 hours. Values were normalised to Gapdh and then expressed as fold change compared to either level of proliferation (b and c) or to siScrambled conditions (f-i). Data is presented as mean \pm SEM from three experiments $(n=3$ mice). Asterisks $(*)$ denote significance at $\mathrm{p}<0.05$ compared to control conditions (either proliferating myoblasts or siScrambled cohorts), using one-way ANOVA followed by the TukeyKramer post-hoc ( $a$ and $b$ ) or a 2-tailed paired student t-test (f-i). 
Ortuste Quiroga et al.

\section{Effects of Piezo1 downregulation on Piezo2}

Using RT-qPCR analysis, the expression of Piezo2 in Piezol-downregulated conditions was measured in both EDL and soleus primary cells (Figures 1f-i). Furthermore as Piezol seemed to exert most of its expression during differentiation, we evaluated Piezo2 expression in early differentiated myotubes. This encompassed a 24-hour incubation in differentiation medium at high confluence, followed by siRNA transfection. After overnight incubation with siRNA, the medium was exchanged with fresh differentiation medium and incubated for a further 24 hours before sampling. We again confirmed the reliability in our method of siRNA-mediated knockdown by showing significant reduction of Piezol expression in both EDL- and soleus-derived cells (Figures $1 \mathrm{~h}$ and i). Data from the EDL (Figure 1f) showed that in Piezol-downregulated conditions, the expression of Piezo2 is relatively stable. Soleusderived cells subjected to Piezol-siRNA (Figure 1g) showed a slight trending increase in Piezo2 expression, compared to control-siRNA; however this did not reach statistical significance, indicating that Piezo2 did not provide a compensatory role in the downregulation of Piezol. Though, we must acknowledge that perhaps if the degree of Piezol downregulation was more "potent", Piezo2 may alter its expression in response. Furthermore a prolonged downregulation of Piezo1 may also affect the expression level of Piezo2.

Piezo1 is dispensable for muscle cell proliferation

We next turned our attention to the effects of Piezol downregulation during proliferation. Although we did not see a significant difference in the expression dynamics of Piezol early in the myogenic program that is not to say that the knockdown of this channel 
Ortuste Quiroga et al.

incorporation assays (Figures 2a-d).-Analyses of the proliferation rate in myoblasts derived from EDL (Figures 2a and $\mathrm{b}$ ) showed that the downregulation of Piezol had no significant effect on EdU incorporation, compared to siRNA control conditions. Similarly, the downregulation of Piezol in soleus-derived myoblasts (Figures $2 \mathrm{c}$ and $\mathrm{d}$ ) showed no statistical change in the proliferation rate between Piezol-siRNA-treated cells and control conditions. From these results, we can summarise that on plated cell conditions, downregulation of Piezol does not seem to affect the proliferation capacity of EDL- and soleus-derived myoblasts.

\section{Suppression of Piezo1 does not inhibit the onset of the myogenic program}

Our next aim was to investigate whether knockdown of Piezol would alter the capacity of cells to enter into the differentiation phase of the myogenic program. siRNA-transfected cohorts were cultured in differentiation medium for 24 hours. This early differentiation phase was analysed by immunolabelling of the myogenic regulatory factor (MRF) Myogenin. Quantification was performed by calculating the proportion of Myogenin-positive myoblasts relative to the number of DAPI counterstained nuclei (Figures 2e-h). Analysis of EDLderived myoblasts (Figures $2 \mathrm{e}$ and $2 \mathrm{f}$ ) showed no significant difference in the relative proportion of myogenin-positive cells between control-siRNA and Piezol-siRNA treated conditions-Similarly, soleus-derived cells (Figures $2 \mathrm{~g}$ and $2 \mathrm{~h}$ ) did not show a significant difference in the relative proportion of myogenin-positive cells in samples treated with Piezol-siRNA, compared to control-siRNA. From these results, we may suggest that the downregulation of Piezol does not seem to have any effects on the ability for myoblasts to enter the differentiation phase of the myogenic program. However, the question remained 
bioRxiv preprint doi: https://doi.org/10.1101/2020.09.27.315242; this version posted November 22, 2020. The copyright holder for this preprint (which was not certified by peer review) is the author/funder, who has granted bioRxiv a license to display the preprint in perpetuity. It is made available under aCC-BY-NC-ND 4.0 International license.

Ortuste Quiroga et al.

406

whether the continuous knockdown of this receptor would affect the fusion and maturation of

407 myotubes. To answer this, we turn to myotube analyses.

408 
bioRxiv preprint doi: https://doi.org/10.1101/2020.0927 315242. this version posted November 22, 2020. The copyright holder for this preprint (which was not certified by peer review) is the author/funder, who has granted bioRxiv a license to display the preprint in perpetuity. It is made available under aCC-BY-NC-ND 4.0 International license.

Ortuste Quiroga et al.

409

a)
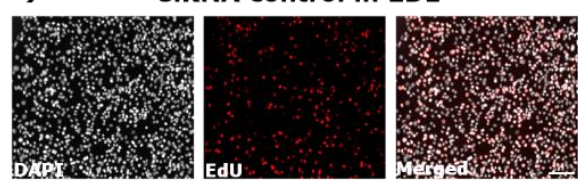

SiRNA Piezo1 in EDL
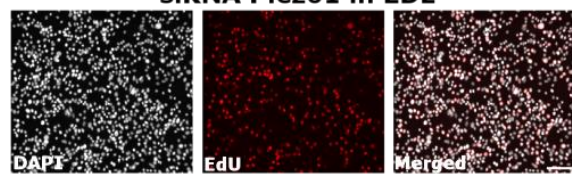

b) Proportion of EdU incorporation in EDLderived myoblasts

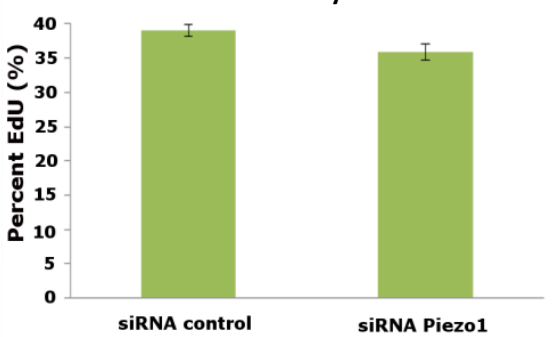

\section{Proliferation}

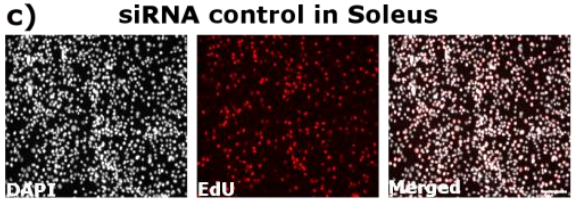

SiRNA Piezo1 in Soleus

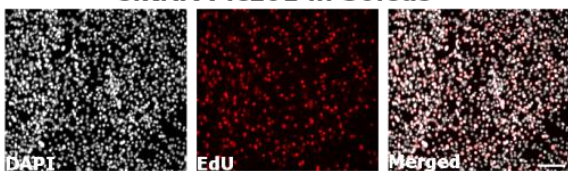

d) Proportion of EdU incorporation in Soleus-derived myoblasts

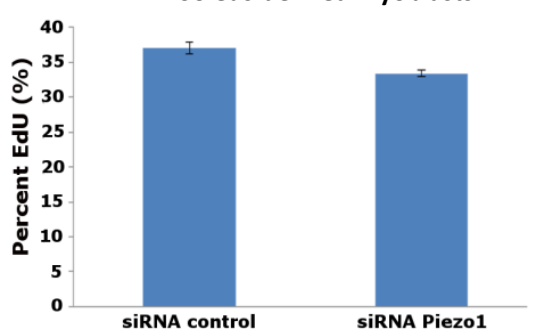

Day 1 differentiation

e)
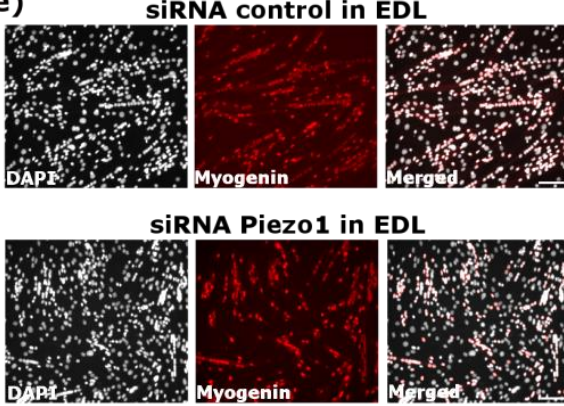

f)

Proportion of Myogenin positive cells in EDL-derived myoblasts

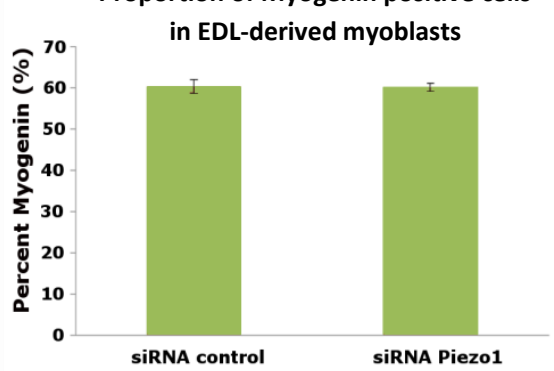

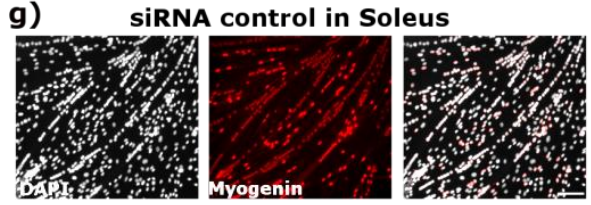

SiRNA Piezo1 in Soleus

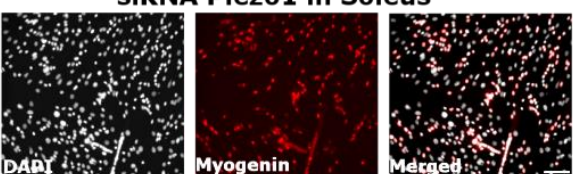

h) Proportion of Myogenin positive cells in Soleus-derived myoblasts

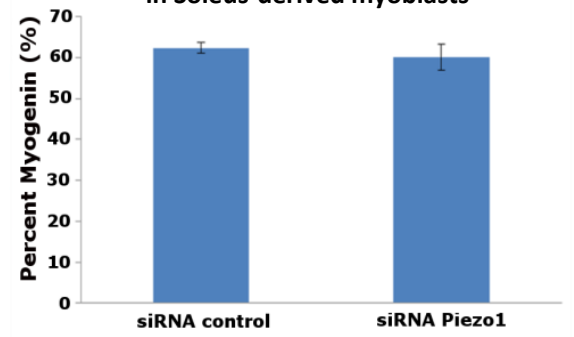

424 Figure 2. Piezo1 knockdown does not affect proliferation rate nor early entry into differentiation.

425 a) and c) Representative images of EDL and soleus primary-derived myoblasts, transfected with 10nM of siRNAcontrol

426 (siScrambled) or siRNA-Piezo1. Following overnight incubation, the medium was changed with fresh proliferation

427 medium and cells were incubated for a further 24 hours, and then subjected to a 2-hour pulse with EdU (red panels).

428 DAPI counterstained nuclei shown in black and white panels. Scale bar is $100 \mu \mathrm{m}$. b) and d) Proportion of EdU-

429 incorporated cells relative to total (DAPI) cell count. e) and g) Representative images of EDL and soleus muscle-derived 
430 myoblasts transfected with 10nM of control-siRNA (siScrambled) or Piezol-siRNA. Following overnight incubation,

431 cells were incubated for a further 24 hours, immunolabelled for Myogenin (red panels) and counterstained with DAPI

432 (black and white panels). Scale bar is $100 \mu \mathrm{m}$. f) and h) Percentage proportion of Myogenin positive cells relative to total

433 nuclei. Data is represented as percentages. Data is mean \pm SEM from three experiments $(\mathrm{n}=3$ mice). *: significant at $\mathrm{p}$

$434<0.05$ compared to siScrambled conditions using a 2-tailed paired student t-test.

435

436 
Ortuste Quiroga et al.

Piezo1 regulates myocytes fusion

Piezol-siRNA-treated cells were cultured in differentiation medium for 3 days post transfection. By this timepoint myoblasts fuse to neighbouring differentiating myoblasts and begin to upregulate the sarcomeric motor protein $\mathrm{MyHC}$, which is expressed in the forming does this allow a visual representation of myotube formation, but it can also be used to quantify the level of fusion that has occurred - the fusion index.

The data derived from the EDL-derived cells (Figure 3a), showed a clear difference between control-siRNA and Piezol-siRNA conditions, with the latter displaying much fewer myotubes. Indeed, we observed a significant $(\mathrm{p}<0.05)$ reduction of fusion index in PiezolsiRNA conditions compared to control conditions (Figure 3b). These findings were also translated to soleus-derived cells. From the myotube images (Figure 3c), we observed smaller myotubes when Piezol was downregulated. This was confirmed by the significant reduction in myocytes fusion (Figure 3d) in Piezol-siRNA conditions compared to control conditions $(\mathrm{p}<0.05)$.

A potential concern of the data in Figures $3 a-d$, is the fact that cells were first transfected in proliferating conditions (i.e. in proliferation medium) before being switched to differentiate; and although the initial analysis of EdU- and myogenin-positive cells did not reveal a change post siRNA-mediated knockdown of Piezol, that is not to say that the initial knockdown in proliferating conditions did not alter the behaviour of the channel and the subsequent formation of myotubes. Thus, in order to address this, we carried out the transfection of Piezol-siRNA in differentiating conditions. This encompassed a 24-hour incubation period in differentiation medium. By this point primary derived myoblasts seeded at high confluency, already begin to show myotube formation in the relatively small 96-well 
Ortuste Quiroga et al.

461

462

463

464

465

466

467

468

469

470

471

472

473

474

475

476

plates. These early formed myotubes were then subjected to Piezol-siRNA transfection in differentiation medium. Following overnight incubation, the transfection mixture was exchanged with fresh differentiation medium. Myotubes were subsequently analysed 3 days post the initial Piezol-siRNA transfection (Figures 3e-h).

Downregulation of Piezol in EDL-derived myotubes (Figures 3e and 3f), showed a significant reduction $(\mathrm{p}<0.05)$ in the fusion index, compared to control-siRNA conditions. The soleus muscle (Figures $3 \mathrm{~g}$ and $3 \mathrm{~h}$ ) also showed a reduction in the ability of myoblasts to fuse in Piezol-siRNA-treated conditions, compared to control-siRNA cohorts $(\mathrm{p}<0.05)$. In summary, the downregulation of the Piezo1 channel both in forming and maturing myotubes significantly reduces the ability of cells to fuse into new or existing myotubes. This response seems to be unique to this phase of the myogenic program as downregulation of Piezol does not affect proliferation or early entry into differentiation.

These thought-provoking results demand further exploration of; 1) the mechanisms of Piezo1-mediated fusion, 2) other fusion regulators, and 3) the downstream signalling pathways of the Piezo1 channel which enables correct myogenic fusion. 
bioRxiv preprint doi: https://doi.org/10.1101/2020.0927 315242. this version posted November 22, 2020. The copyright holder for this preprint (which was not certified by peer review) is the author/funder, who has granted bioRxiv a license to display the preprint in perpetuity. It is made available under aCC-BY-NC-ND 4.0 International license.

Ortuste Quiroga et al.

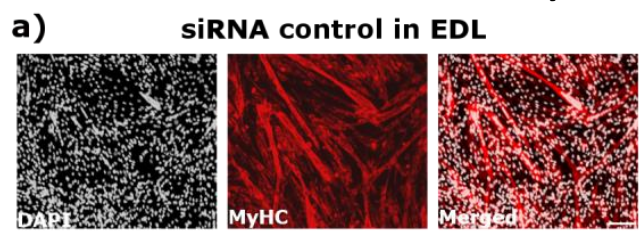

Myotube Formation
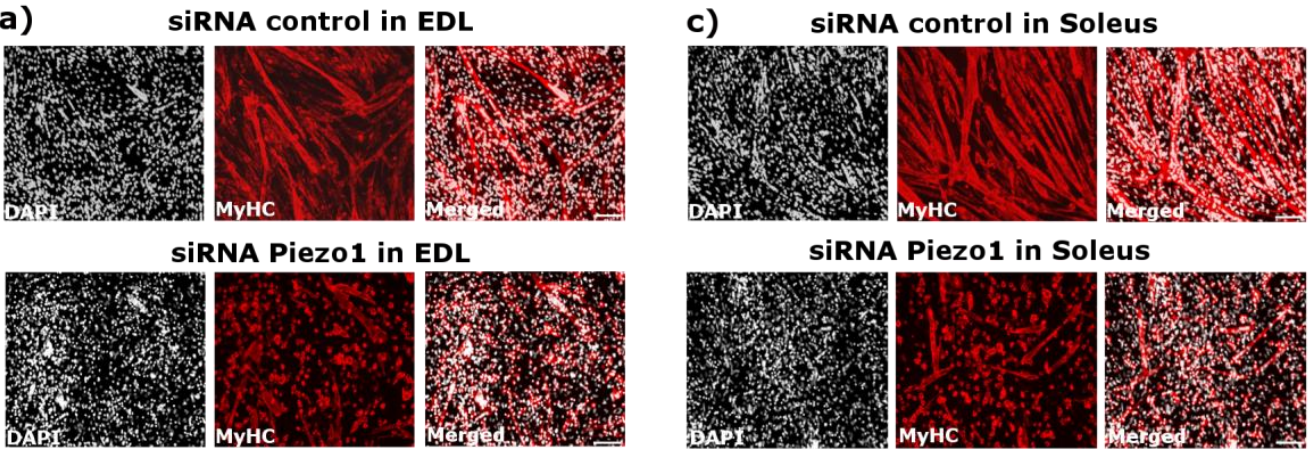

b)

Fusion index in EDL-derived myotubes
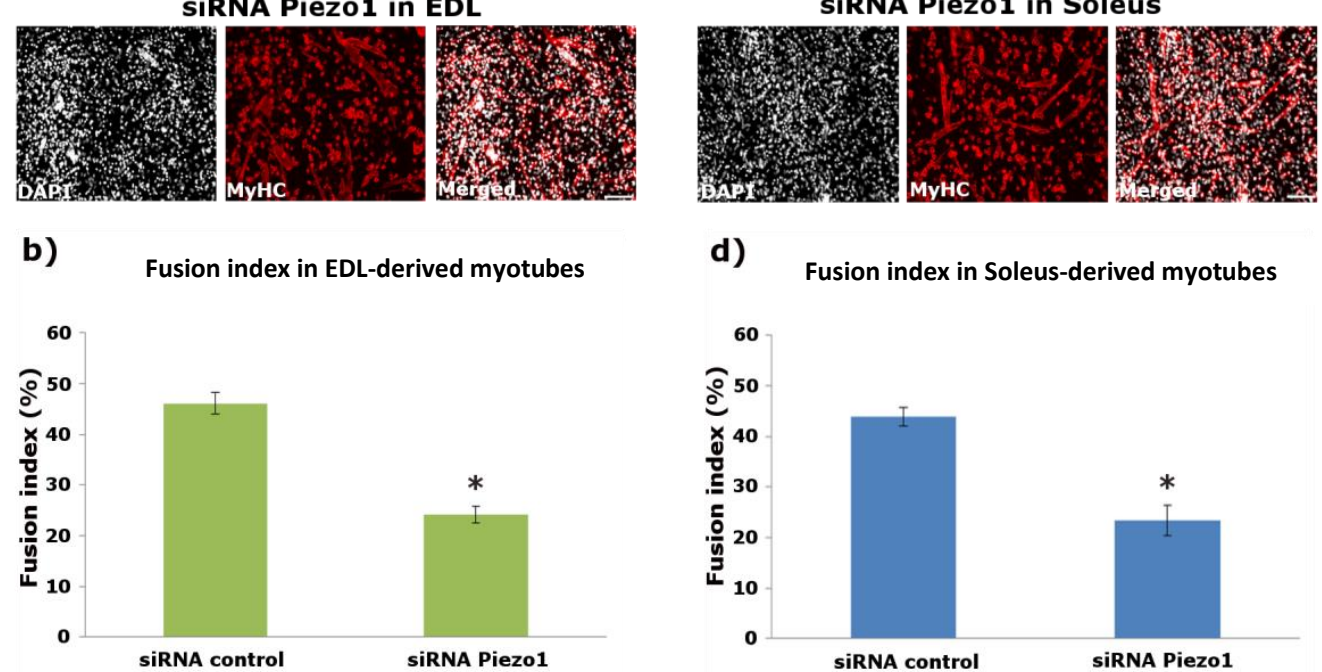

d)

Fusion index in Soleus-derived myotubes

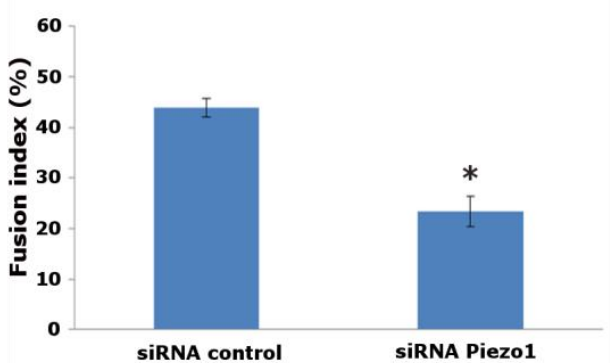

e)

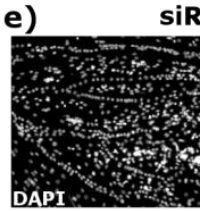

SiRNA Piezo1 in EDL

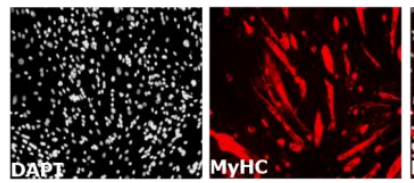

f)

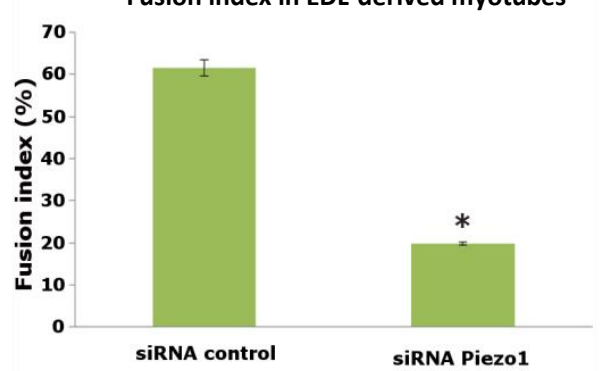

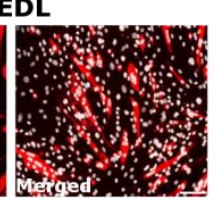

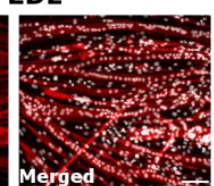

Myotube Maturation

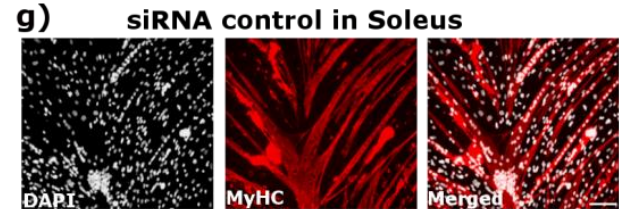

SiRNA Piezo1 in Soleus
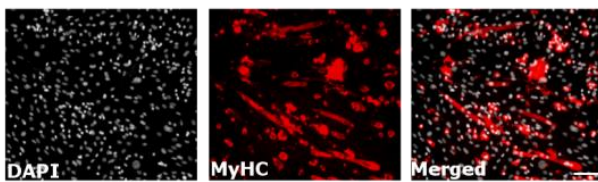

h)

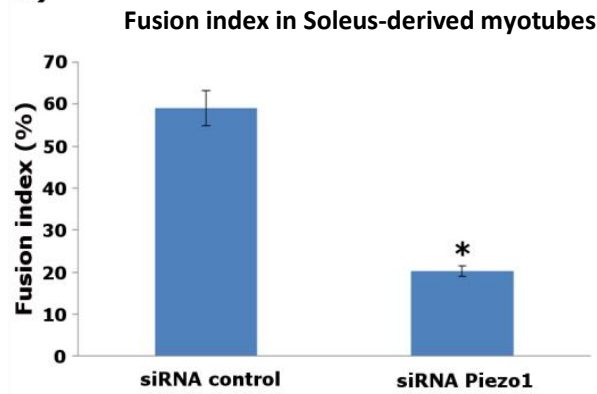

Figure 3. Piezo1 knockdown reduced fusion indexes during myotube formation and maturation. 


\section{Ortuste Quiroga et al.}

500 Representative images of EDL and soleus muscle-derived. Early forming myotubes were transfected with 10nM of

501 control-siRNA (siScrambled) or Piezol-siRNA. Following overnight incubation, cells were incubated for a further 72 hours. immunolabelled for Myosin heavy chain (MyHC) (red panels) and counterstained with DAPI (black and white panels). Scale bar is $100 \mu \mathrm{m}$. f) and h) Bar graphs display the fusion index. Data is mean \pm SEM from three experiments for myotube formation data $(n=3$ mice $)$ and four experiments for myotube maturation data $(n=4$ mice $)$. 
Ortuste Quiroga et al.

\section{Piezo1 and actin remodelling}

Previous research places Piezol regulation as a key modulator of this cytoskeletal protein [20,21]. In skeletal muscle actin plays a pivotal role not only in myoblast fusion but myotube alignment to attachment sites [22]. Therefore, a putative mechanism(s) of Piezo1 is the regulation of cytoskeletal structures, including filamentous actin (f-actin). As part of our analyses, we examined f-actin intensity using fluorescently labelled phalloidin as a proxy to evaluate its accumulation, and thus the extent of cytoskeletal reorganisation during myogenic differentiation in EDL or Soleus derived myoblasts. Piezol-siRNA-treated cells showed a significant decrease in the accumulation of f-actin compared to control-siRNA (Figure S2). in f-actin intensity during any of the incubation timepoints or concentrations (Figure S3).

Further, high concentration and relatively longer incubation time of Yoda1, suggestive of an over-activation of Piezo1, showed a significant decrease in f-actin in EDL (100 $\mu \mathrm{M}$; 4-hour incubation) and soleus (100 $\mu \mathrm{M} ; 30$-min, 1- and 4-hour incubation) derived myotubes. These findings suggest that cytoskeletal organisation may be affected by Piezo1 activity; however our methods of measuring such changes lacked resolution. For example, f-actin stress fibre formation as well as filopodia extension in response to Piezo1 manipulation should be quantified under higher magnification.

\section{Lack of Piezol affects the expression of fusogenic genes}

An ever-increasing topic of interest within the skeletal muscle field is the role fusion proteins play. The recently discovered fusion protein Myomaker and its associated 
Ortuste Quiroga et al.

centre of myogenic fusion research [23-26]. Thus, we were curious to examine the effects

Piezol may have on these fusion proteins. Piezol was first downregulated in EDL- and

soleus-derived myoblasts. Cells were then collected and RT-qPCR against Myomaker and Myomixer was carried out and compared to control-siRNA conditions (Figure 4a-d). EDLderived cells (Figure 4a) showed that the expression of Myomaker is significantly reduced in Piezol-siRNA treated cells $(\sim 43 \%)$, compared to control-siRNA conditions $(\mathrm{p}<0.05)$. In Piezol-downregulated conditions, Myomixer (Figure 4b) showed a trend to decrease, compared to control-siRNA, $(\mathrm{p}=0.0505)$. In soleus-derived cells (Figure 4c), downregulation of Piezol significantly lowered the expression of Myomaker ( 30\%), compared to controlsiRNA ( $\mathrm{p}<0.05)$. Similar to the EDL, we observed a trend for Myomixer to decrease post

540 Piezol knockdown (Figure 4d, p>0.05). Taken together, these results show a potential interplay between the stretch induced Piezo1 channel and the fusion protein gene Myomaker actively employs mechanisms which lower Myomaker expression which in turn reduce

544 myogenic fusion or whether this is an indirect effect of another Piezol governed event.

545 Answering these queries may have implication in skeletal muscle regeneration and muscular dystrophies. 
a)

549

550

551

552

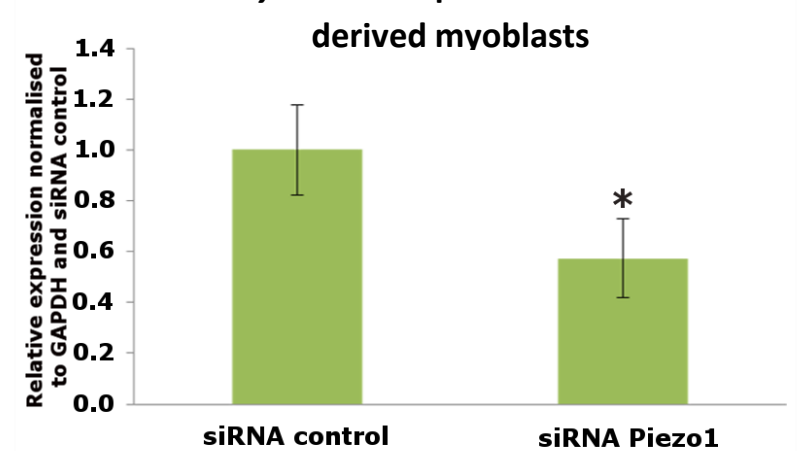

553

c)

Myomaker expression in

554

555

556

557

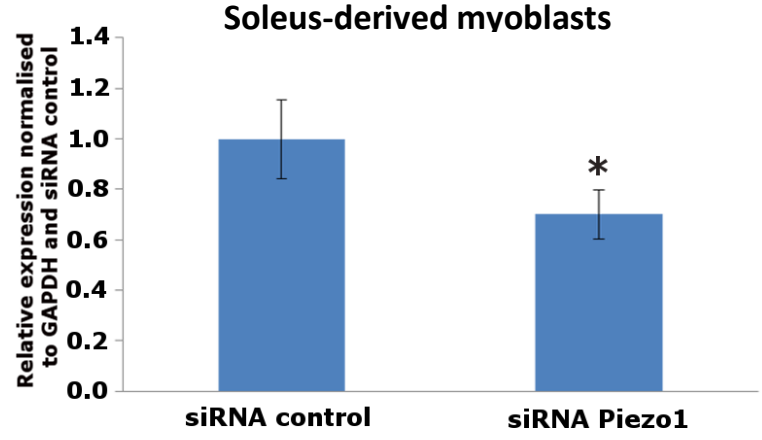

b)

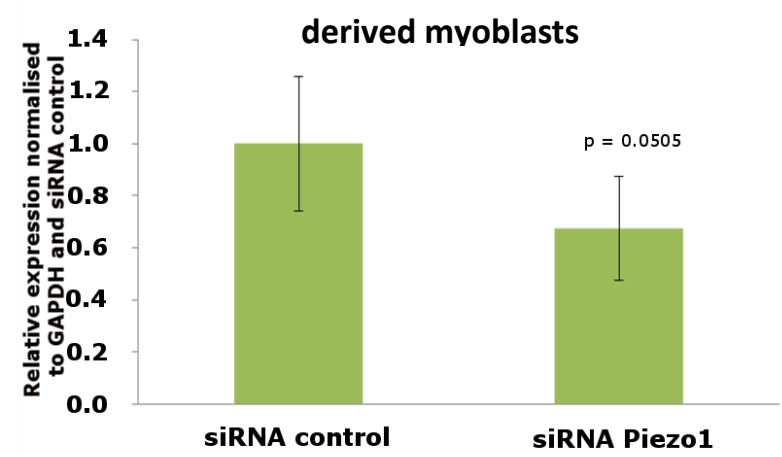

Figure 4. Myomaker expression is reduced in Piezo1-downregulated conditions

Relative fold changes in expression of EDL-derived myoblasts ( $a$ and $b$ ) and soleus-derived myoblasts ( $c$ and d). Cells were transfected with $10 \mathrm{nM}$ of either control-siRNA (siScrambled) or Piezol-siRNA. After overnight incubation, cells incubated for a further 24 hours. The expression of the fusogenic protein genes Myomaker and Myomixer were then using a 2-tailed paired student t-test. 
Ortuste Quiroga et al.

\section{$\mathrm{Ca}^{2+}$ influx in Piezo1 downregulated myotubes}

The Piezol channel permeates $\mathrm{Ca}^{2+}$ influx at a greater preference than other cations

$\left(\mathrm{Na}^{+}, \mathrm{K}^{+}\right.$and $\left.\mathrm{Mg}^{2+}\right)$ [5]. $\mathrm{Ca}^{2+}$ is itself a crucial regulatory and signalling molecule of the myogenic program as well as skeletal muscle contraction and various intracellular events.

Thus, a logical query to pursue was to see whether selective downregulation of Piezol

hinders or blunts the influx of $\mathrm{Ca}^{2+}$ in cultured myotubes exposed to stretch. To achieve this we used customised stretch silicon bio-chambers [16]. Myoblasts from both EDL and soleus muscles were transfected with either Piezol-siRNA or control-siRNA (siScrambled). The transfection mixture was then removed, and samples were cultured in differentiation medium for 3 days. The resulting myotubes were then subjected to incremental bouts of stretch. membrane-permeant fluorescent $\mathrm{Ca}^{2+}$ indicator (Figure 5). conditions. In EDL-derived myotubes for example (Figures 5a and 5c), a 6\% stretch significantly increased $\left[\mathrm{Ca}^{2+}\right] \mathrm{i}$ influx compared to $0 \%$ stretch controls $(\mathrm{p}<0.05)$. An increase in $\left[\mathrm{Ca}^{2+}\right] \mathrm{i}$ was also observed in EDL-derived myotubes at $9 \%$ stretch conditions $(\mathrm{p}<0.05)$. A $9 \%$ stretch bout also showed significantly higher $\left[\mathrm{Ca}^{2+}\right]$ i, compared to $3 \%$ stretch conditions $(\mathrm{p}<0.05)$. Soleus-derived myotubes (Figures $5 b$ and $5 d$ ) at stretch conditions of $6 \%$ and $9 \%$, showed a significant increase in $\left[\mathrm{Ca}^{2+}\right] \mathrm{i}$, compared to $0 \%$ stretch conditions $(\mathrm{p}<0.05)$. Soleusderived myotubes in $9 \%$ stretch conditions also demonstrated higher $\left[\mathrm{Ca}^{2+}\right] \mathrm{i}$, compared to $3 \%$ stretch bouts $(\mathrm{p}<0.05)$. Neither EDL- nor soleus-derived myotubes at the relatively lower stretch bout of $3 \%$, showed a significant increase in $\left[\mathrm{Ca}^{2+}\right] \mathrm{i}$, compared to $0 \%$ stretch controls. changes in $\left[\mathrm{Ca}^{2+}\right] \mathrm{i}$ in cultured myotubes. 
Ortuste Quiroga et al.

Turning our attention to the effects of Piezol downregulation, we witnessed a striking suppression in $\left[\mathrm{Ca}^{2+}\right] \mathrm{i}$ in response to stretch compared to control-siRNA (Figures 5a and 5b).

myotubes (Figure 5c). Indeed neither a 6\%, nor a 9\% stretch could elicit a significant increase in $\left[\mathrm{Ca}^{2+}\right]$ i. In soleus-derived cells (Figure 5d), only Piezol-downregulated myotubes at the 9\% stretch condition, were able to show increased $\left[\mathrm{Ca}^{2+}\right] \mathrm{i}$, compared to $3 \%$ stretch counterparts $(\mathrm{p}<0.05)$.

Comparison of $\left[\mathrm{Ca}^{2+}\right] \mathrm{i}$ between control-siRNA and Piezol-siRNA cohorts at each stretch condition further cemented the remarkable reduction of $\left[\mathrm{Ca}^{2+}\right] \mathrm{i}$ in Piezol downregulated conditions in response to stretch (Figure 5c). EDL-derived myotubes at 3\%

601 stretch in Piezol-siRNA conditions, show a suppression in $\left[\mathrm{Ca}^{2+}\right] \mathrm{i}$ compared to controlsiRNA counterparts $(\mathrm{p}<0.05)$. This is also true for $6 \%$ cohorts, with Piezol-siRNA transfected EDL myotubes displaying a lower $\left[\mathrm{Ca}^{2+}\right] \mathrm{i}$, compared to control-siRNA $(\mathrm{p}<0.05)$. At $9 \%$ stretch we continued to see a significant suppression of increase in $\left[\mathrm{Ca}^{2+}\right] \mathrm{i}$ in PiezolsiRNA treated conditions, compared to control-siRNA at the same stretch bout $(\mathrm{p}<0.05)$. In persisted. Indeed, a 3\% stretch showed a lower $\left[\mathrm{Ca}^{2+}\right]$ i in Piezol-downregulated conditions, compared to $3 \%$ stretch in control-siRNA. At $6 \%$ stretch, increase in $\left[\mathrm{Ca}^{2+}\right] \mathrm{i}$ is again blunted in Piezol-downregulated cohorts, compared to siRNA controls $(\mathrm{p}<0.05)$. This is further demonstrated at 9\% stretch bouts, with Piezol-downregulated myotubes showing a reduced increase in $\left[\mathrm{Ca}^{2+}\right] \mathrm{i}$, compared to control-siRNA- $(\mathrm{p}<0.05)$. The suppression of increase in

$612\left[\mathrm{Ca}^{2+}\right]$ i following Piezol downregulation may be a key factor in the reduction of fusion observed in Figure 3. 
bioRxiv preprint doi: https://doi org/10.1101/2020 09.27.315242; this version posted November 22, 2020. The copyright holder for this preprint (which was not certified by peer review) is the author/funder, who has granted bioRxiv a license to display the preprint in perpetuity. It is made available under aCC-BY-NC-ND 4.0 International license.

Ortuste Quiroga et al.

a)

EDL
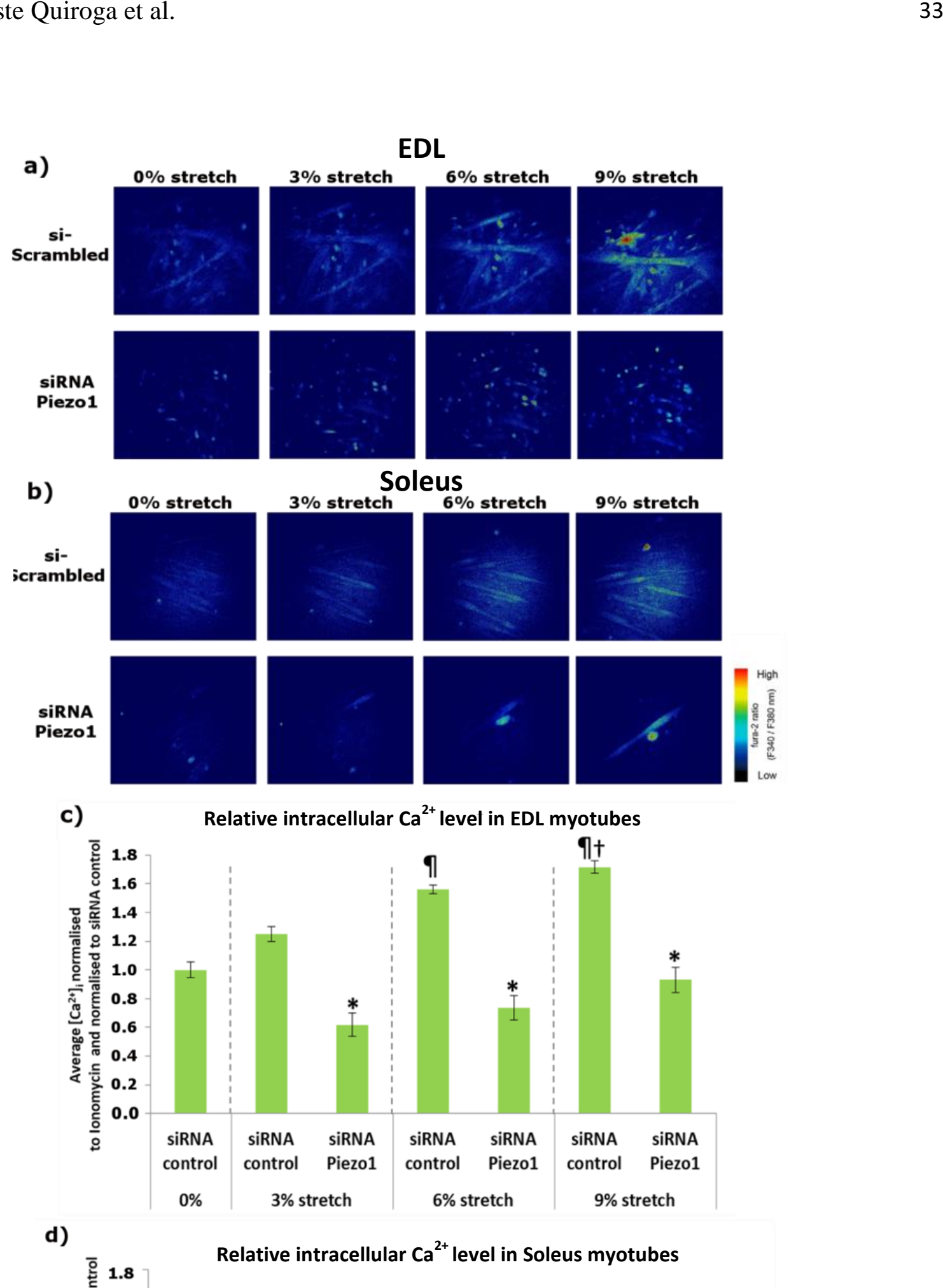

9\% stretch

618
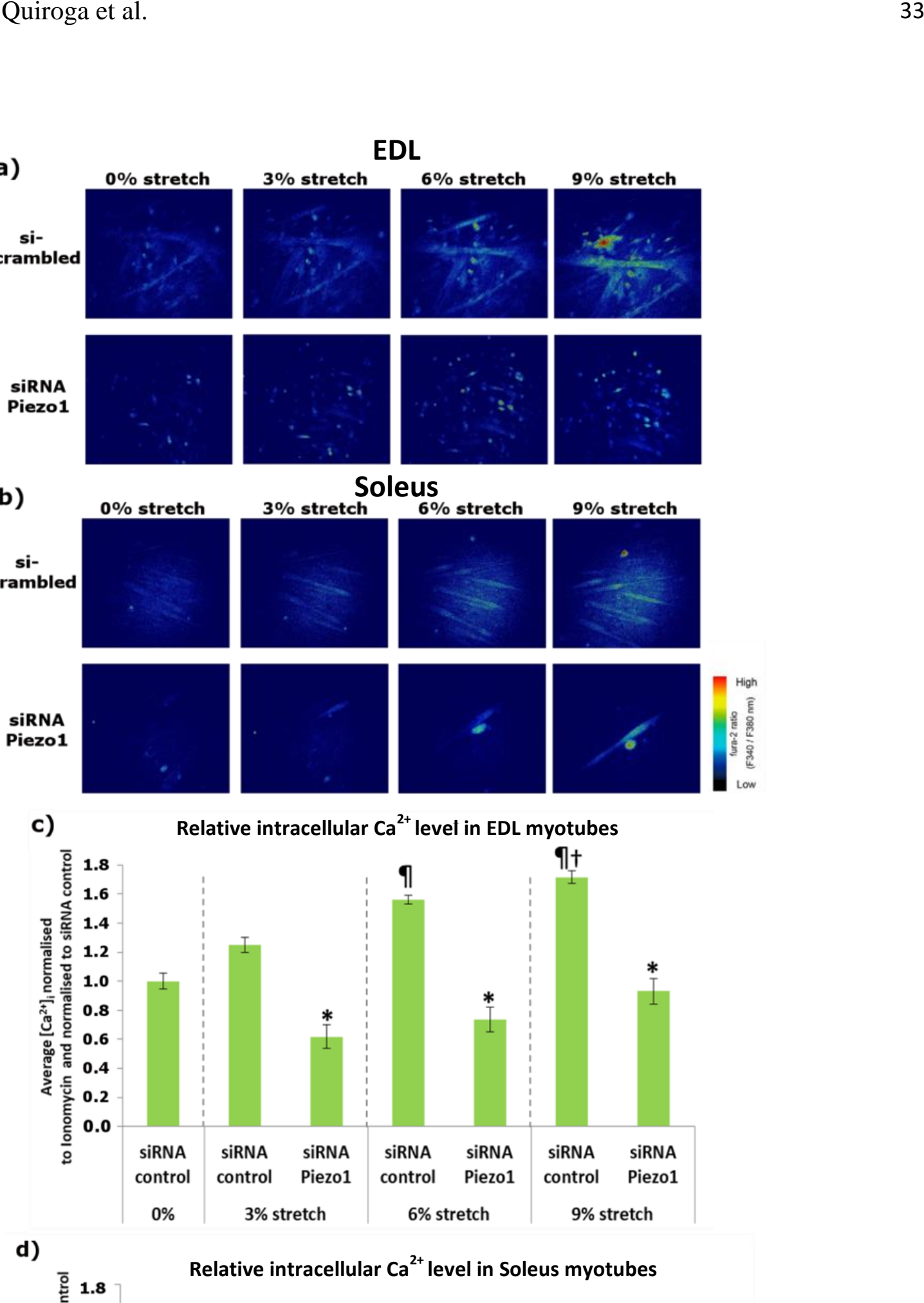

b)

Soleus

620

sijcrambled

$0 \%$ stretch
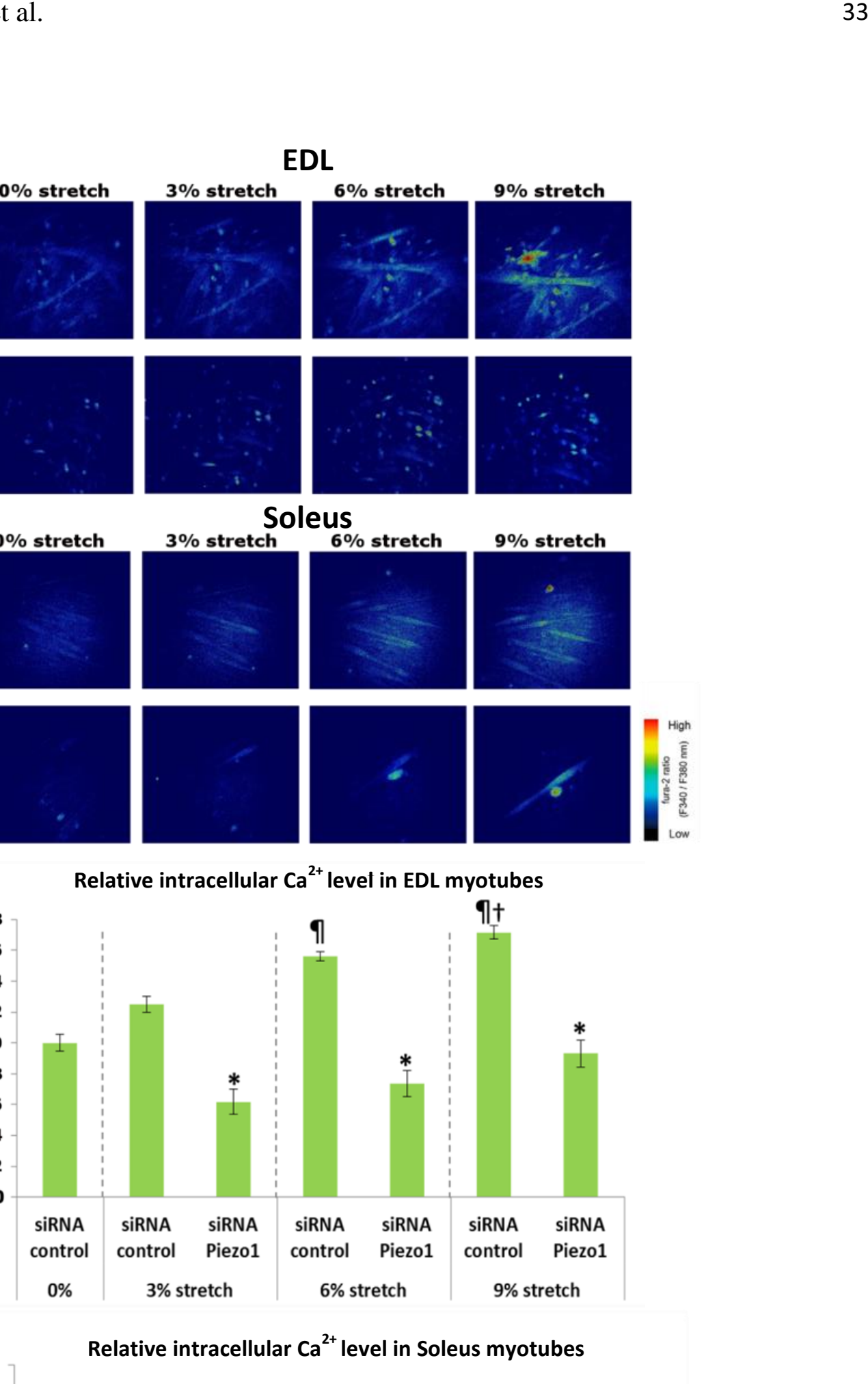

622

SIRNA Piezo1
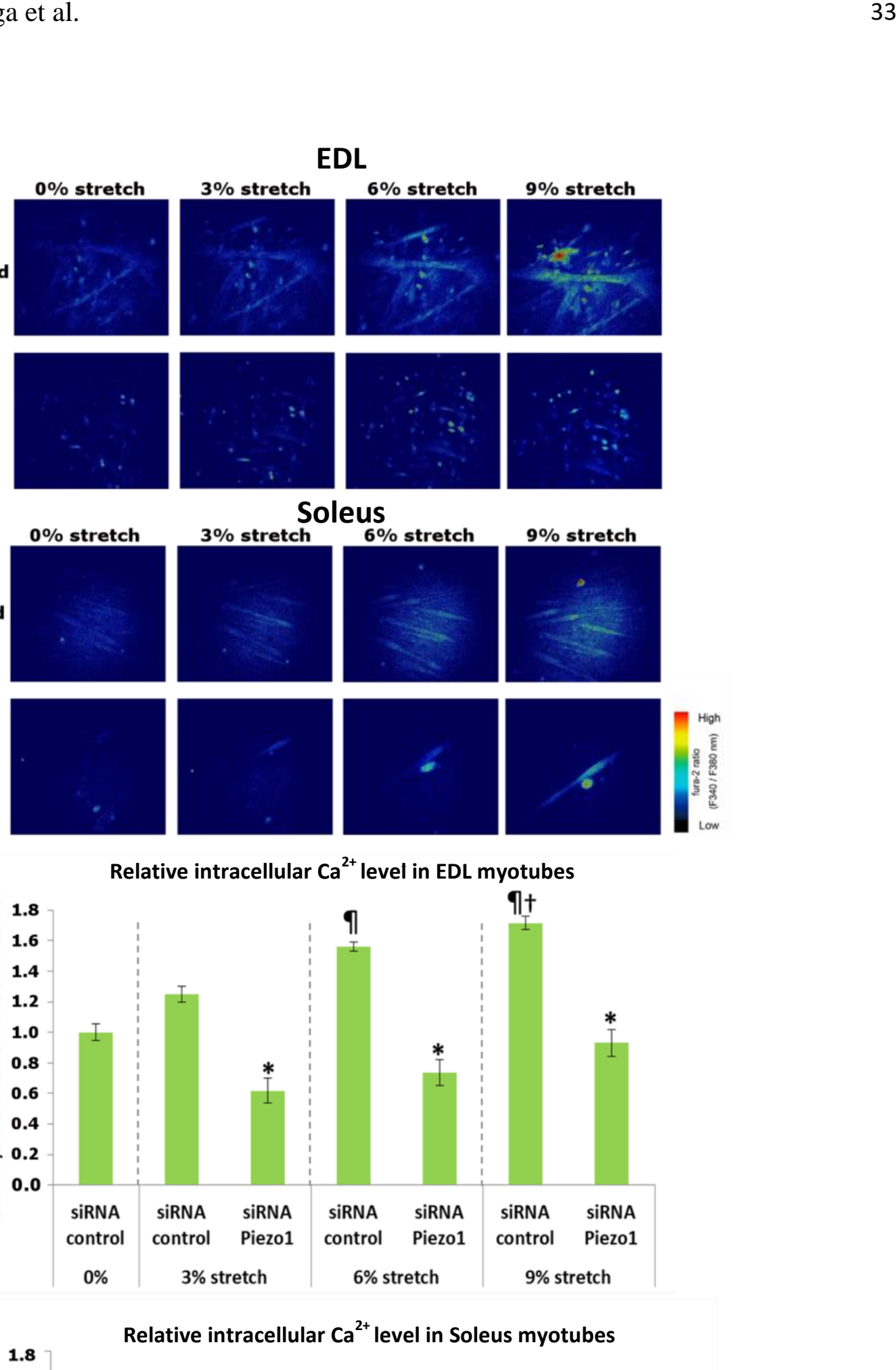

c)

Relative intracellular $\mathrm{Ca}^{2+}$ level in EDL myotubes

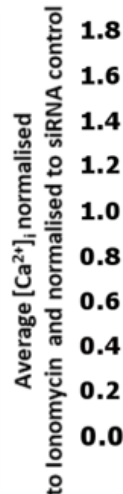

d)

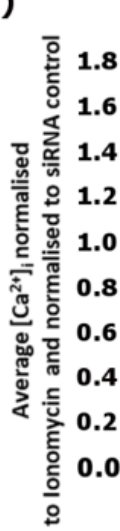

\begin{tabular}{c|cc}
\hline siRNA & siRNA & siRNA \\
control & control & Piezo1
\end{tabular}

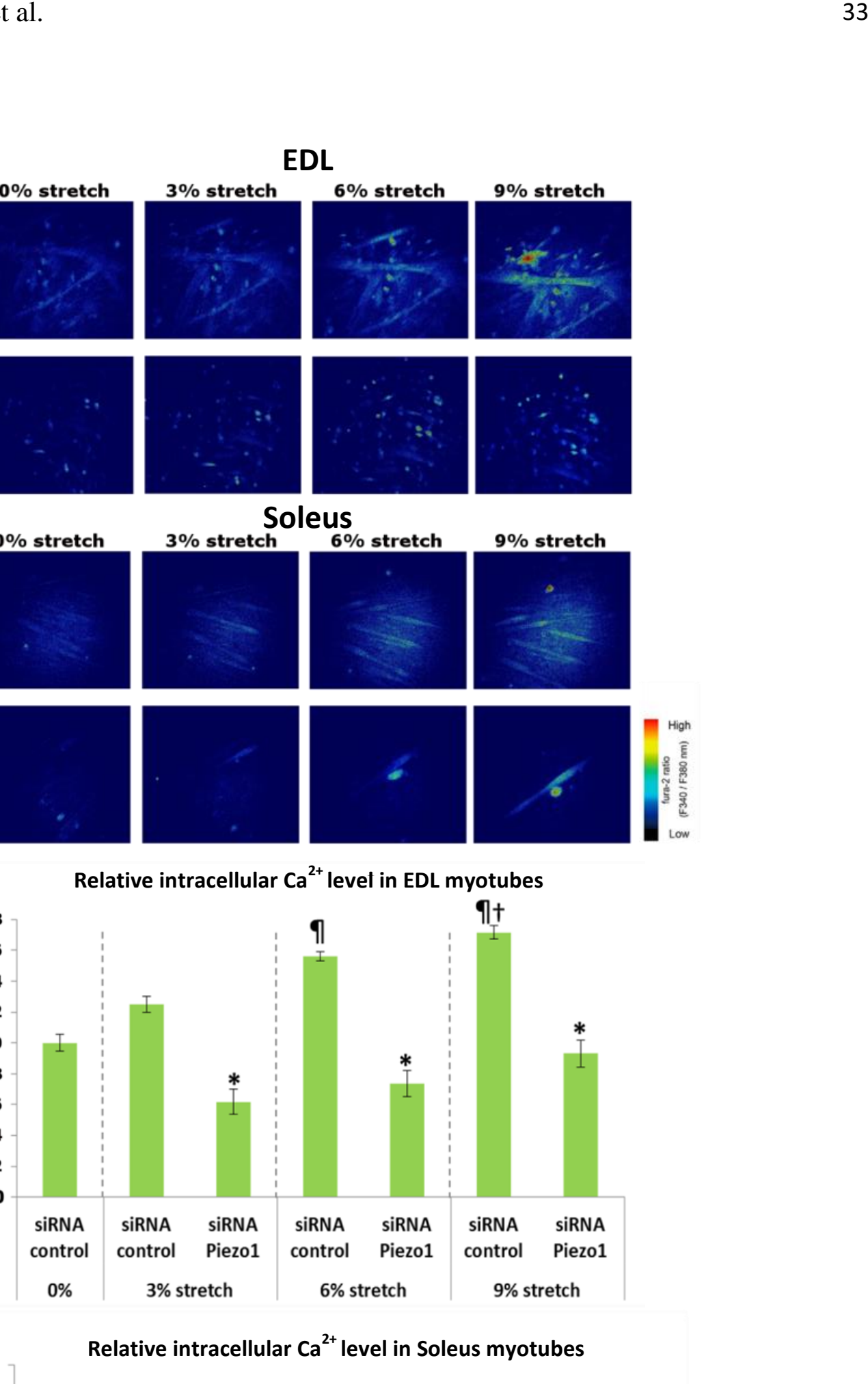

$0 \%$

$3 \%$ stretch

$6 \%$ stretch

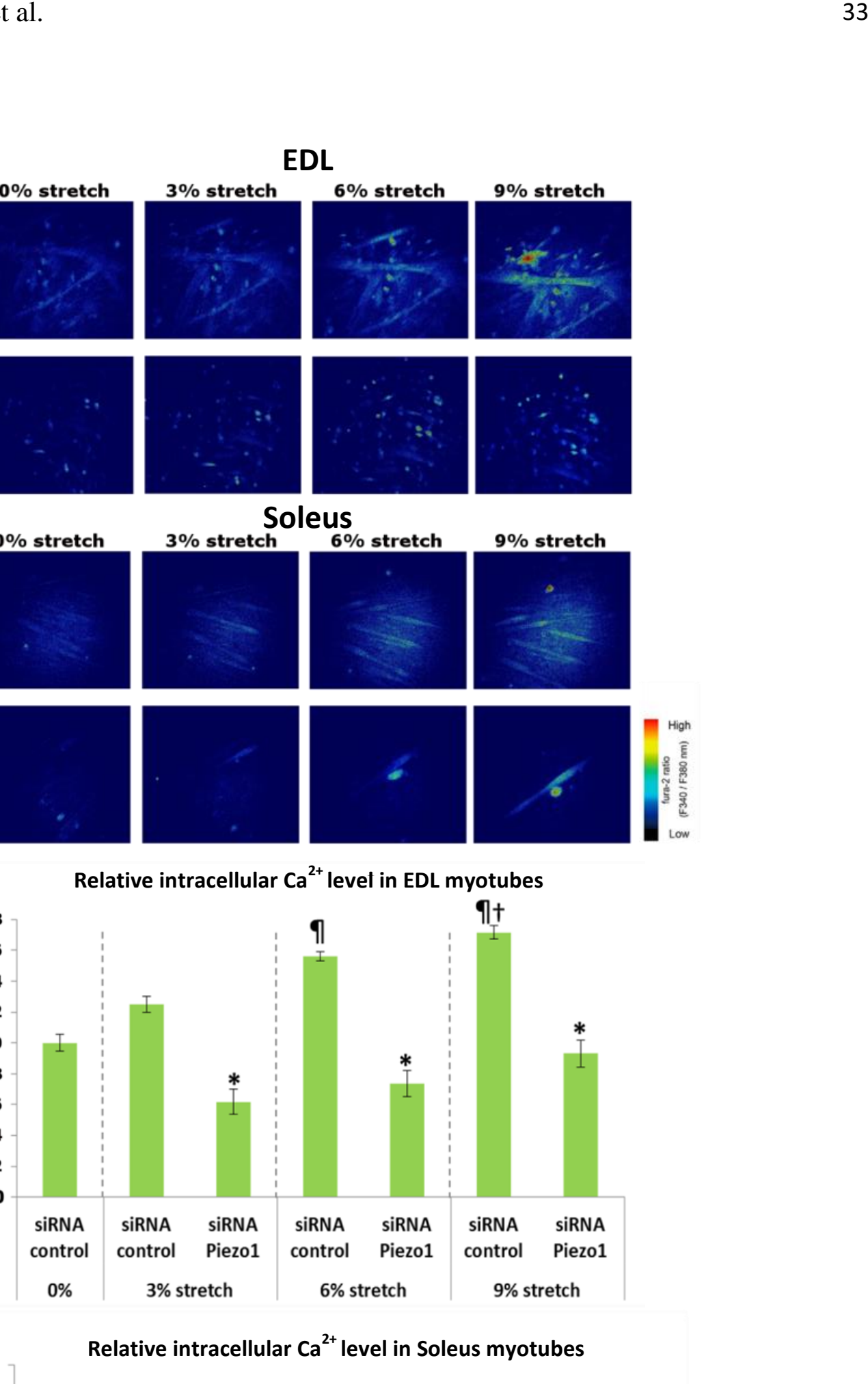

Relative intracellular $\mathrm{Ca}^{2+}$ level in Soleus myotubes 
bioRxiv preprint doi: https://doi org/10.1101/2020.09.27.315242; this version posted November 22, 2020. The copyright holder for this preprint (which was not certified by peer review) is the author/funder, who has granted bioRxiv a license to display the preprint in perpetuity. It is made available under aCC-BY-NC-ND 4.0 International license.

Ortuste Quiroga et al.

Figure 5. Piezo1 downregulation inhibits stretch-mediated increase of intracellular $\mathrm{Ca}^{2+}$.

634 a) and b) Representative images of intracellular $\mathrm{Ca}^{2+}$ imaging in EDL and soleus-derived myotubes. Myoblasts were transfected with $10 \mathrm{nM}$ of either control-siRNA (siScrambled) (top panels) or Piezol-siRNA (bottom panels). After overnight incubation, cells were incubated for a further 72 hours. Fura 2-AM was administered to myotubes followed

637 by a 30-min incubation time. Stretch was then applied at 3\% (0.3 mm), 6\% (0.6 mm) and 9\% (0.9 mm) for $1 \mathrm{~min}$ 638 followed by a 1-min resting period in between. Ionomycin at $5 \mu \mathrm{M}$ was then applied. Side vertical bar shows Fura 2639 AM ratio emittance from low to high. c) and d) Average changes in the intracellular $\mathrm{Ca}^{2+}$ level ([Ca $\left.\left.{ }^{2+}\right] \mathrm{i}\right)$ calculated by 640 difference between base and peak pixel value) normalised to ionomycin and control-siRNA at 0\% stretch. Values are 641 mean \pm SEM from three experiments $(n=3$ mice). *: Significant difference at $\mathrm{p}<0.05$ compared to control-siRNA at 642 each condition. II: Significant difference at $\mathrm{p}<0.05$ compared to $0 \%$ stretch counterparts. $\uparrow:$ Significant difference at $p$ $643<0.05$ compared to $3 \%$ stretch counterparts. F: Significant difference at $\mathrm{p}<0.05$ compared to $9 \%$ stretch in Piezol644 siRNA conditions using one-way ANOVA followed by the Tukey-Kramer post-hoc. 
Ortuste Quiroga et al.

The next phase of the investigation demanded we look at the effects of Piezo1 activation in myotube fusion and $\mathrm{Ca}^{2+}$ influx. This was achieved using the Piezol specific agonist Yoda1. Our preliminary findings showed that a 24-hour incubation of myotubes with all the chosen Yoda1 concentrations significantly inhibited myotube maturation (data not shown). Thus the subsequent experiments set out to test a series timepoints with our chosen Yoda1 concentrations, in order to uncover a potential "sweet-spot" between agonist concentration, incubation time and our hypothesised increase in myogenic fusion. Early forming myotubes (cells incubated in differentiation medium for 24 hours at high cell confluency in 96-well plates) were subjected to varying timepoints and concentrations of Yoda1. After each allocated timepoint, the agonist containing medium was removed and replenished with fresh differentiation medium and incubated for a further 2 days (Figure 6). $\mu \mathrm{M}$ and $100 \mu \mathrm{M}$ incubation with Yoda1 significantly increased the fusion index compared to DMSO controls. Similarly, soleus-derived myotubes (Figures $6 \mathrm{~b}$ and d) had a significant increase in fusion at $30 \mu \mathrm{M}$ and $100 \mu \mathrm{M}$, compared to DMSO controls $(\mathrm{p}<0.05)$. At the 30 min timepoint, we found that EDL-derived myotubes at Yoda1 concentrations of $30 \mu \mathrm{M}$ had a significantly higher fusion index, compared to DMSO counterparts $(p<0.05)$. Soleus samples incubated for 30 min with Yodal at $10 \mu \mathrm{M}$ and $30 \mu \mathrm{M}$ had a significantly higher fusion index than DMSO controls $(\mathrm{p}<0.05)$. Unlike the 1 min timepoint, a $100 \mu \mathrm{M}$ Yoda1 incubation revealed a reduced fusion index when incubated for 30 minutes. Indeed, both soleus and EDL samples incubated at $100 \mu \mathrm{M}$ beyond the 1 min mark, showed a significant decrease in fusion, compared to DMSO controls. 
Ortuste Quiroga et al.

At the 1-hour incubation timepoint, EDL-derived myotubes exhibited increased fusion

671 derived samples incubated with Yoda1 for 1 hour showed an increase in fusion at $5 \mu \mathrm{M}, 10$

$672 \mu \mathrm{M}$-and $30 \mu \mathrm{M}$, compared to DMSO controls. Four hours of consistent Piezo1 activation

673 showed a significant decrease in fusion of EDL-derived myotubes at a Yoda1 concentration

674 of $30 \mu \mathrm{M}$ compared to DMSO control $(\mathrm{p}<0.05)$. Taken together, we showed that Piezo1

675 activation results in enhanced fusion of myotubes. This is dependent on the duration and

676 potency in which Piezo1 is activated, as too much of either inhibits myotube fusion 
bioRxiv preprint doi: https://doi org/10.1101/2020.09.27.315242; this version posted November 22, 2020. The copyright holder for this

preprint (which was not certified by peer review) is the author/funder, who has granted bioRxiv a license to display the preprint in perpetuity. It is made available under aCC-BY-NC-ND 4.0 International license.

Ortuste Quiroga et al.

a)

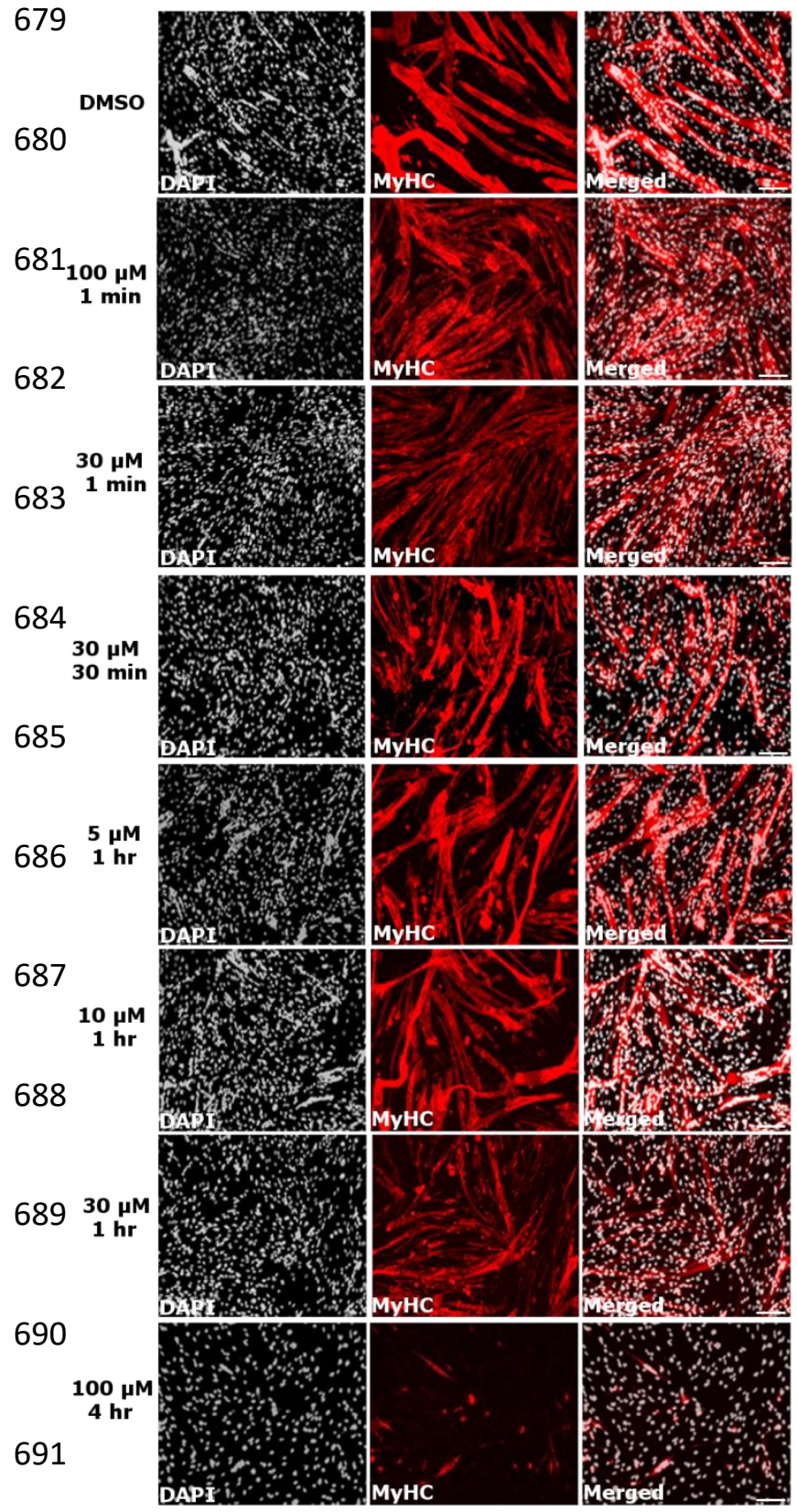

c)

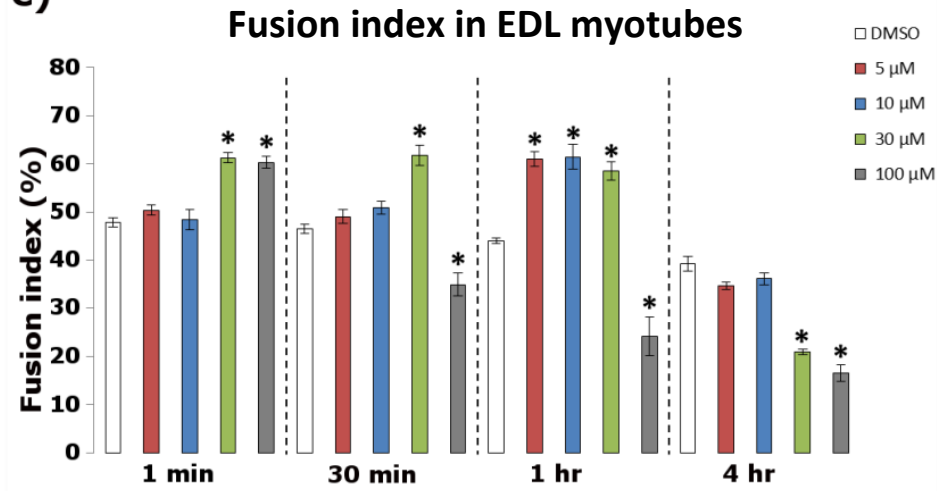

b)

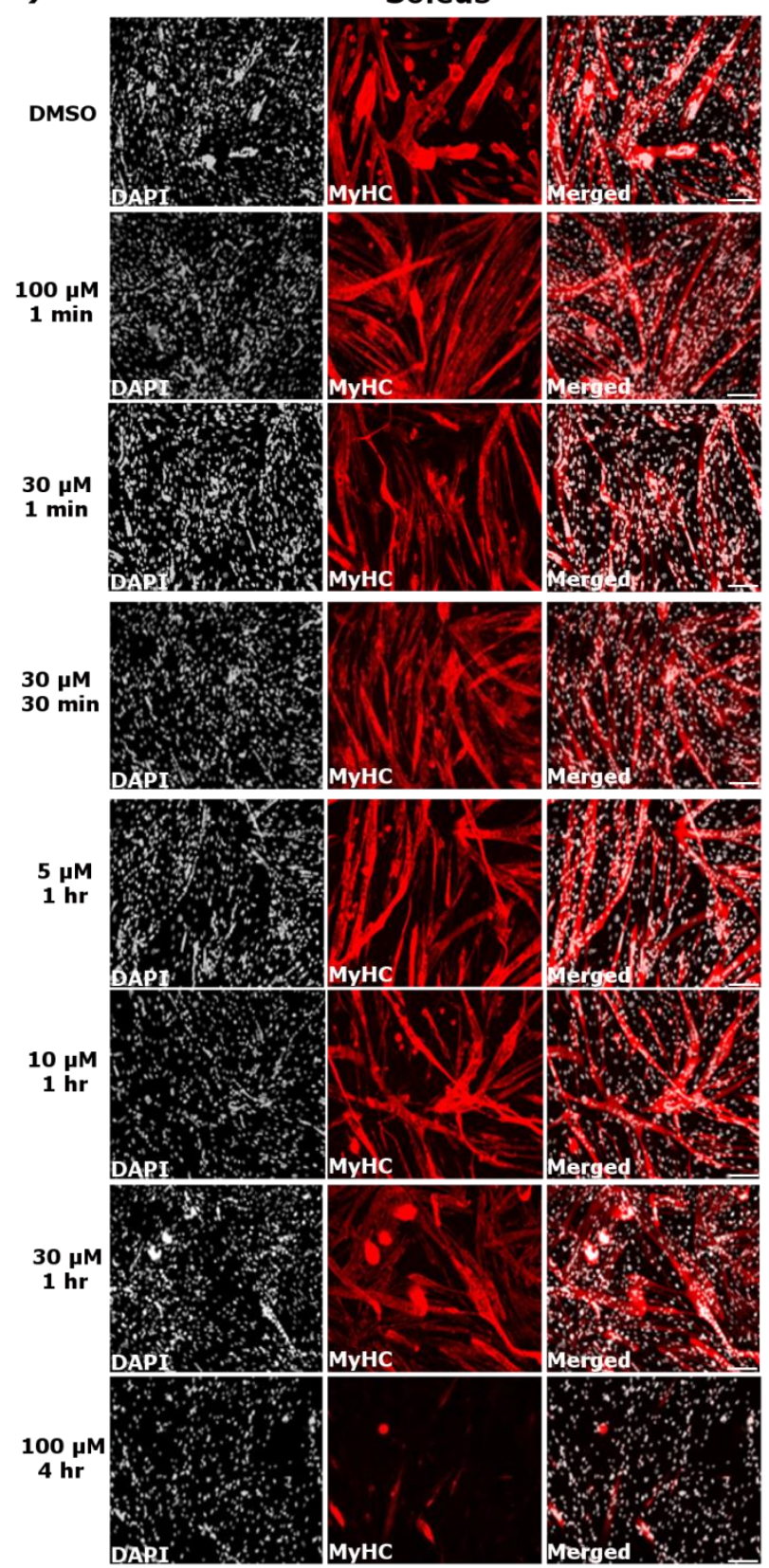

d)

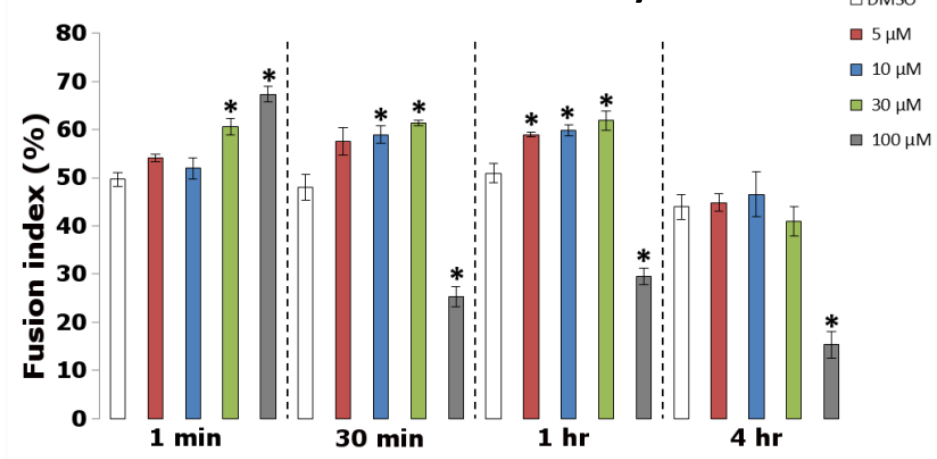


Figure 6. Piezo1 activation increases myogenic fusion.

697 Early forming myotubes were administered with either DMSO (control, white bars) or the Piezo1-agonist Yoda1 at the

698 following concentration: $5 \mu \mathrm{M}$ (red bars), $10 \mu \mathrm{M}$ (blue bars), $30 \mu \mathrm{M}$ (green bars) and $100 \mu \mathrm{M}$ (grey bars). Myotubes

699 were incubated for $1 \mathrm{~min}, 10 \mathrm{~min}, 30 \mathrm{~min}, 1$ hour and 4 hours. Following the incubation period, the medium was exchanged with fresh reduced medium (without agonist) and myotubes were incubated for a further 2 days. a) and b)

701 Representative images of cohorts at relevant timepoints and concentrations, immunostained for Myosin heavy (MyHC)

702 (red panels) and counterstained with DAPI (black and white panels). Micrographs taken at x20 magnification. Scale

703 bar is $100 \mu \mathrm{m}$. Bar graphs display fusion index in c) EDL and d) soleus-derived myotubes from each time point and

704 concentration variables. Values are mean \pm SEM. * indicates significance at $\mathrm{p}<0.05$ using one-way ANOVA tests followed by the Tukey-Kramer post-hoc. $n=3$ mice. 
Ortuste Quiroga et al.

707

708

709

710

711

712

713

714

715

716

717

718

719

720

721

722

723

724

725

726

727

1

\section{Myotube width and Piezo1 activation}

We noticed that Yoda1 treated samples that exhibited increased fusion, also appeared to have thinner myotubes. In order to address this, we compared the myotube width of these samples to DMSO controls (Figure 7). Interestingly, both EDL (Figure 7a and b) and soleus derived myotubes (Figure 7c and d) showed reduced myotube width compared to controls. We further subdivided myotube width into bins of $5 \mu \mathrm{m}$ increments and compared the distribution of Yodal treated myotubes at the 1 min incubation with $100 \mu \mathrm{M}$ concentration to DMSO controls. Both EDL (Figure 7e) and soleus derived myotubes (Figure 7f), showed that Yoda1 treated cells, on average, have a greater proportion of smaller myotubes. In contrast DMSO controls have a higher distribution of lager myotubes. A similar pattern was observed in the rest of the Yoda1 treated cohorts that displayed increased fusion (Figure S5). Taken together it appears that increased fusion post Yodal administration comes at the cost of myotube syncytial maturation. 
bioRxiv preprint doi: https://doi org/10.1101/2020.09 27 315242. this version posted November 22, 2020. The copyright holder for this preprint (which was not certified by peer review) is the author/funder, who has granted bioRxiv a license to display the preprint in perpetuity. It is made available under aCC-BY-NC-ND 4.0 International license.

Ortuste Quiroga et al.

a)

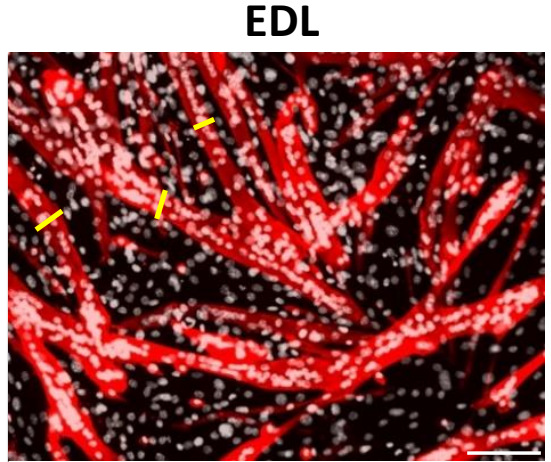

732

733

734

735

736

737

738

739

740

741

\section{e)}

742

743

744

745

746

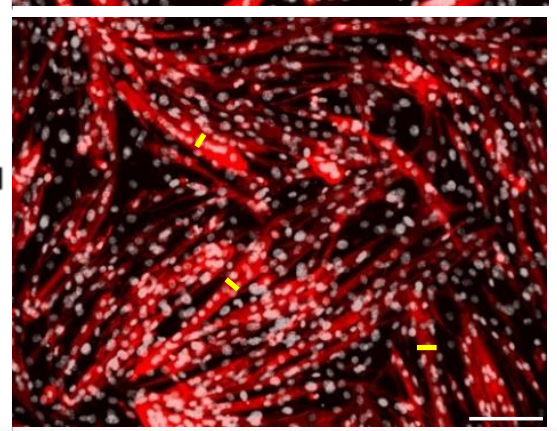

b) Myotube width in EDL
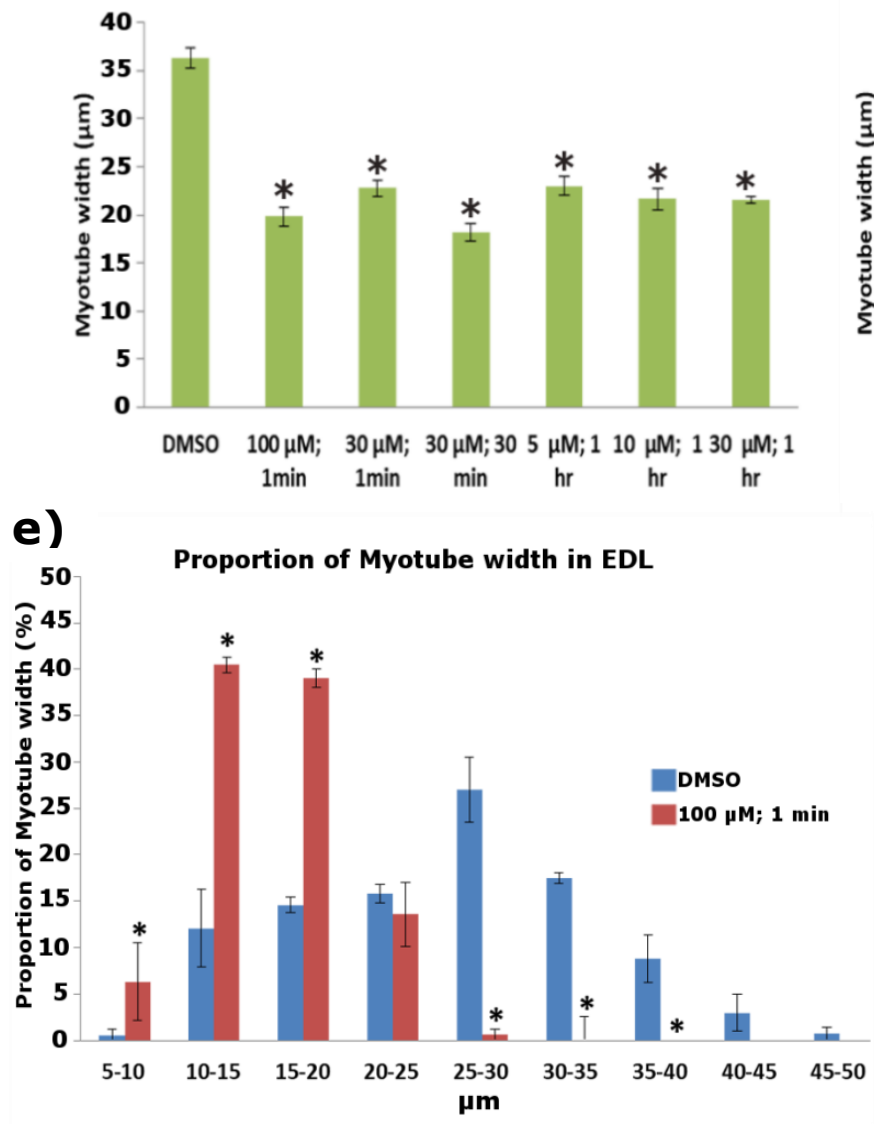

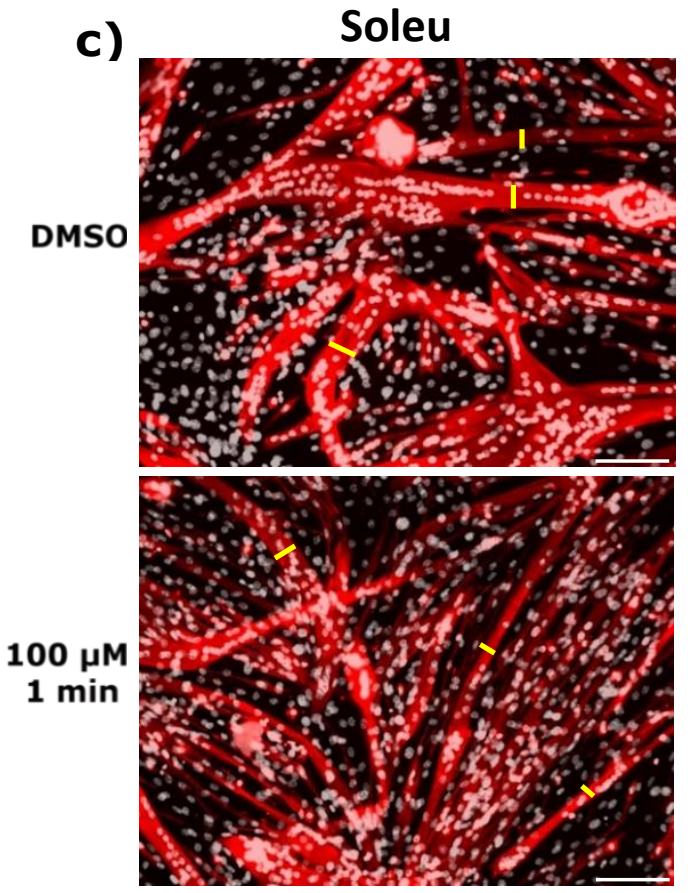

d)

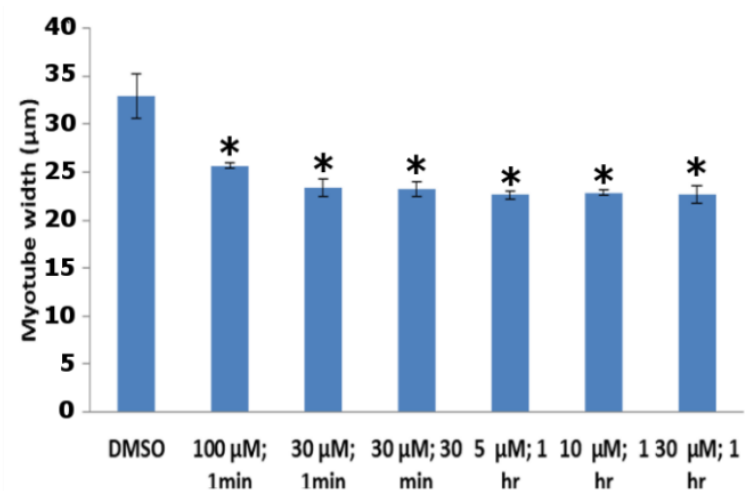

f)

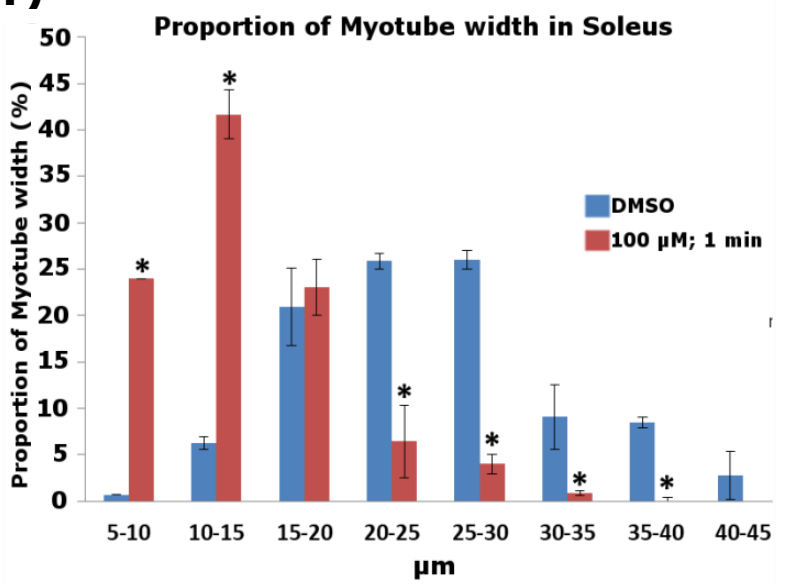


bioRxiv preprint doi: https://doi org/10.1101/2020.09.27.315242; this version posted November 22, 2020. The copyright holder for this preprint (which was not certified by peer review) is the author/funder, who has granted bioRxiv a license to display the preprint in perpetuity. It is made available under aCC-BY-NC-ND 4.0 International license.

Ortuste Quiroga et al.

Figure 7. Yoda1-mediated activation of Piezo1 decreases myotube width.

748 Representative images of a) EDL and c) soleus derived myotubes immunolabelled for MyHC. We measured the width of the myotubes to quantify potential differences between Piezo1-activated cohorts (lower panels) and DMSO controls (upper panels). This was achieved by taking three independent points within a field of view (yellow bars) and measuring the distance from one side of the myotube to the other. Example pictures from DMSO controls and $100 \mu \mathrm{M}$ of Yodal incubated for 1 min are displayed. Scale bar is $100 \mu \mathrm{m} . \mathrm{b}$ ) and d) Average myotube width in DMSO and

753 Piezo1 activated samples. e) and f) Proportion of myotube width in EDL and soleus-derived myotubes. Myotube width

754 was divided into incremental bins of $5 \mu \mathrm{m}$ and represented as percentages relative to the total number of myotubes counted. Data is mean \pm SEM from three experiments data $(n=3$ mice $)$ *: Significant at $\mathrm{p}<0.05$ compared to DMSO controls conditions using one-way ANOVA followed by the Tukey-Kramer post-hoc. Please note that only cohorts which showed increased myogenic fusion in both EDL and soleus myotubes from Figure 6 are displayed. 
Ortuste Quiroga et al.

759

\section{$\mathrm{Ca}^{2+}$ influx and Piezo1 activation}

The next aim of was to determine whether Piezol activation leads to increased influx of $\mathrm{Ca}^{2+}$ in cultured myotubes. Using the customised stretch silicon bio-chambers [16], we cultured myotubes derived from both EDL and soleus muscle. We then divided the samples into two groups; those given the Piezo1 agonist Yoda1 (at $30 \mu \mathrm{M}$ ) and those without. The chambers were then subjected to incremental bouts of stretch, with a minute rest in between each stretch. Throughout the experiment we measured $\left[\mathrm{Ca}^{2+}\right] \mathrm{i}$ (Figure 8 ).

First of all our results confirmed that increasing stretch amplitude results in higher $\left[\mathrm{Ca}^{2+}\right] \mathrm{i}$, which means a greater influx of $\mathrm{Ca}^{2+}$, in both EDL- and soleus-derived myotubes (Figures 8c and 8d). Indeed under control conditions (without Yoda1), EDL-derived myotubes subjected to $6 \%$ stretch and $9 \%$ stretch show a higher $\left[\mathrm{Ca}^{2+}\right] \mathrm{i}$, compared to $0 \%$ stretch controls $(p<0.05)$. Similarly, soleus-derived myotubes at a stretch of $6 \%$ and $9 \%$ exhibited a higher $\left[\mathrm{Ca}^{2+}\right] \mathrm{i}$, compared to $0 \%$ stretch counterparts $(\mathrm{p}<0.05)$. Notably EDL- and soleus-derived myotubes under $3 \%$ of stretch showed no significant difference in $\left[\mathrm{Ca}^{2+}\right]$, compared to $0 \%$ stretch counterparts. In contrast when Yodal was administered prior to stretch, we find that the apparent "threshold" for $\mathrm{Ca}^{2+}$ influx is decreased. Indeed at $3 \%$ stretch bouts (Figures 8c and 8d), we found that both EDL- and soleus-derived myotubes showed a significantly higher $\left[\mathrm{Ca}^{2+}\right] \mathrm{i}$ compared to their respective $0 \%$ stretch control conditions $(\mathrm{p}<0.05)$. Soleus-derived myotubes (Figure $8 \mathrm{~d})$ also exhibited a greater increase in $\left[\mathrm{Ca}^{2+}\right] \mathrm{i}$ post Yoda1 administration in both $6 \%$ and $9 \%$ stretch conditions, compared to $0 \%$ stretch controls $(\mathrm{p}<0.05)$. In a similar pattern (Figure 8c), EDL-derived myotubes at $6 \%$ and 9\% stretch bouts in Yoda1 administered groups, showed a significant increase in $\left[\mathrm{Ca}^{2+}\right] \mathrm{i}$ compared to $0 \%$ controls $(\mathrm{p}<0.05)$. 
Ortuste Quiroga et al.

Comparing the differences between agonist treated groups and those without, at each

783

784

785

786

787

788

789

790

791

792

793

794

795

796

797

798

799

stretch condition, we are able to further demonstrate the increased $\left[\mathrm{Ca}^{2+}\right] \mathrm{i}$ in response to

Piezo1 activation. For example at bouts of 3\% stretch, EDL-derived myotubes treated with

Yoda1 (Figure $8 \mathrm{c}$ ) showed a significantly higher $\left[\mathrm{Ca}^{2+]} \mathrm{i}\right.$, compared to untreated $3 \%$ stretch counterparts. At bouts of $6 \%$ stretch, EDL-derived myotubes given Yoda1 showed a significantly higher $\left[\mathrm{Ca}^{2+}\right] \mathrm{i}$, compared to untreated myotubes $(\mathrm{p}<0.05)$. At $9 \%$ stretch, EDLderived myotubes treated with Yoda1, once again showed a significantly higher $\left[\mathrm{Ca}^{2+}\right] \mathrm{i}$, compared to cohorts without the agonist $(\mathrm{p}<0.05)$. Soleus-derived myotubes at $3 \%$ stretch (Figure $8 \mathrm{~d}$ ), showed that cohorts given Yodal prior, exhibited a higher $\left[\mathrm{Ca}^{2+}\right] \mathrm{i}$, compared to untreated counterparts $(\mathrm{p}<0.05)$. At $6 \%$ stretch the significant difference in $\left[\mathrm{Ca}^{2+}\right] \mathrm{i}$ is also evident, with Yodal treated myotubes showing a higher $\left[\mathrm{Ca}^{2+}\right] \mathrm{i}$, compared to untreated samples $(\mathrm{p}<0.05)$. In line with these findings we showed that soleus-derived myotubes at bouts of $9 \%$ stretch had a higher $\left[\mathrm{Ca}^{2+}\right] \mathrm{i}$ when given Yoda1, compared to myotubes without the Piezo1 agonist $(\mathrm{p}<0.05)$. Taken together, this series of experiments showed that Piezo1 is an essential player in the regulation and function of myotubes. This process seems to involve active modulation of $\mathrm{Ca}^{2+}$ influx. How this leads to increased myotube fusion is an area of great interest and one which demands further research. 
bioRxiv preprint doi: https://doi.org/10.1101/2020.09.27.315242; this version posted November 22, 2020. The copyright holder for this preprint (which was not certified by peer review) is the author/funder, who has granted bioRxiv a license to display the preprint in perpetuity. It is made available under aCC-BY-NC-ND 4.0 International license.

Ortuste Quiroga et al.

a)

0\% stretch

EDL
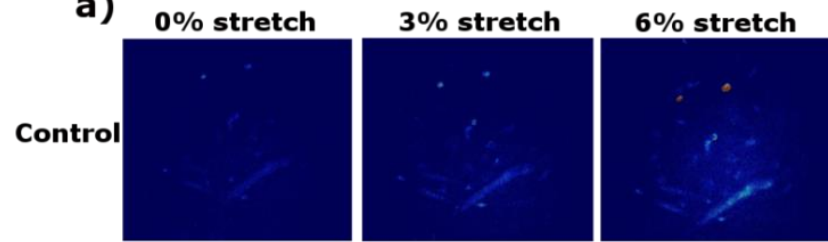

$9 \%$ stretch

806

807
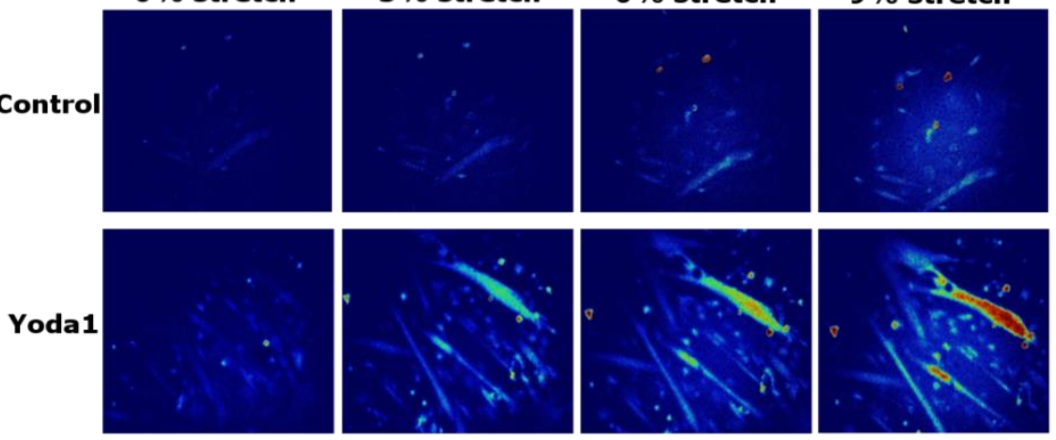

b) $0 \%$ stretch
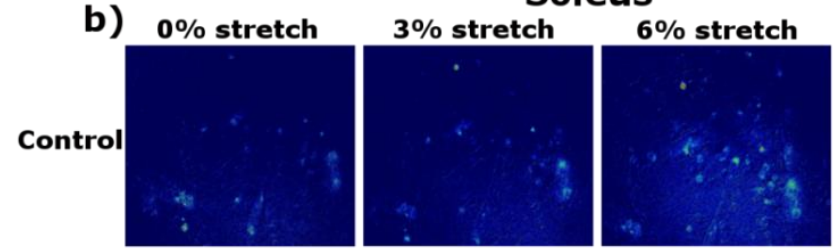

$9 \%$ stretch
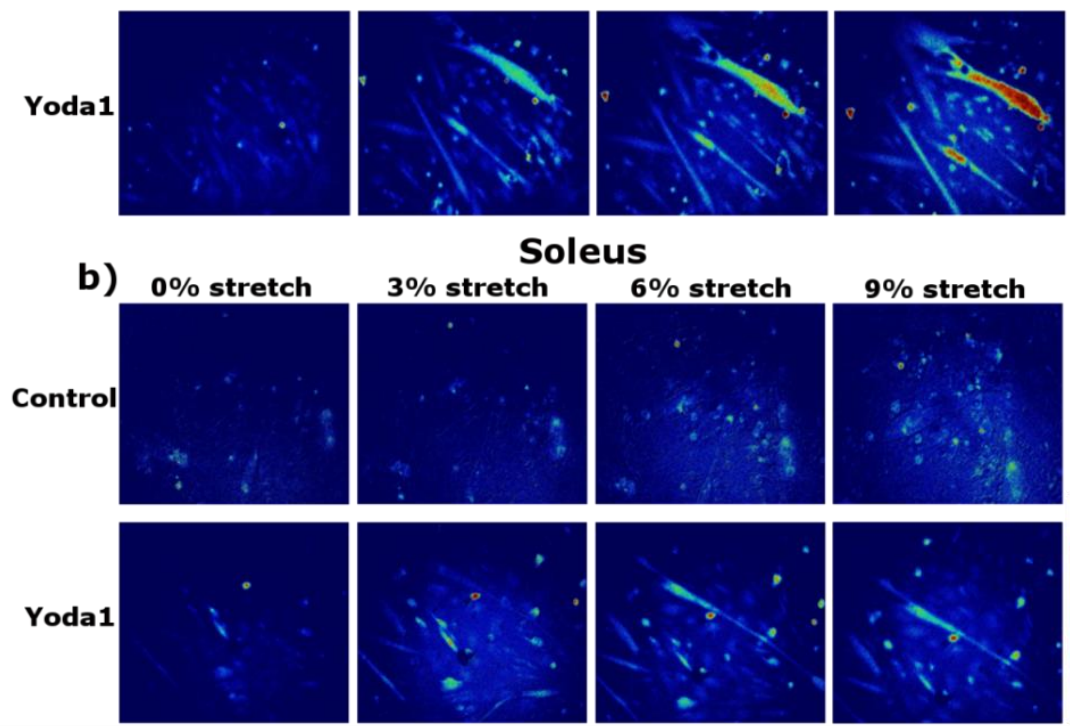

c)

2.0 Relative intracellular $\mathrm{Ca}^{2+}$ level in EDL myotubes

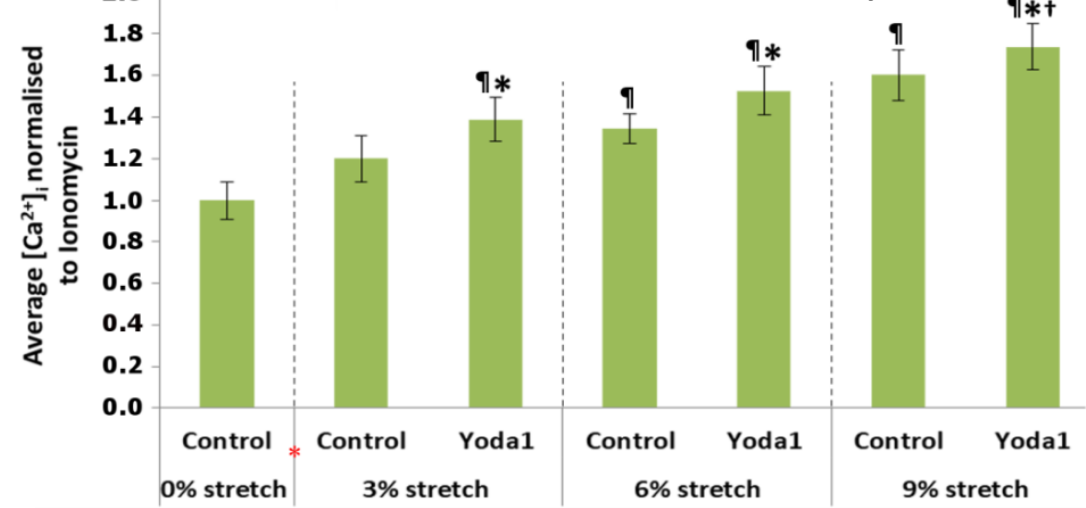

d)

d) $2.0 \quad$ Relative intracellular $\mathrm{Ca}^{2+}$ level in Soleus myotubes

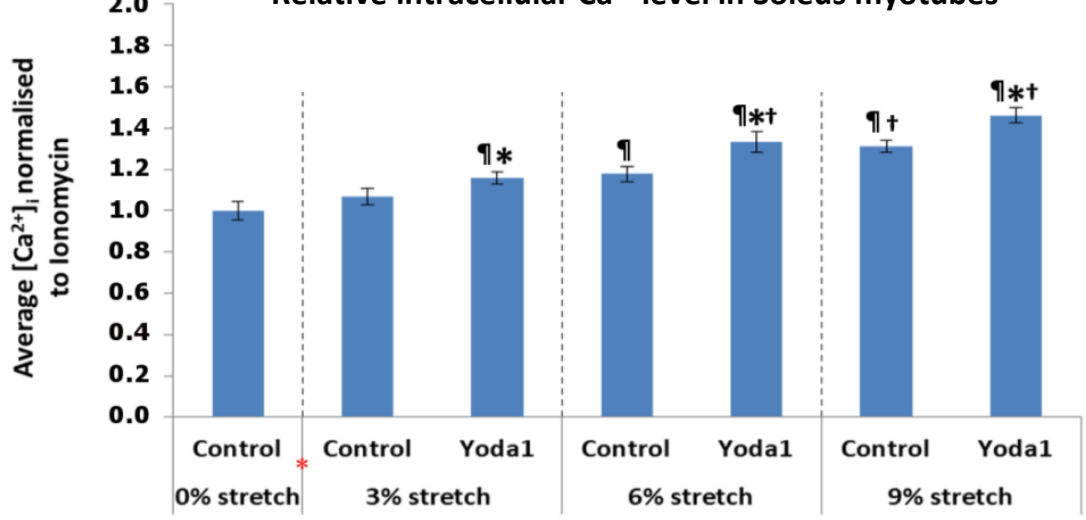

824 Figure 8. Piezo1 activation increases the intracellular $\mathrm{Ca}^{2+}$ level in primary derived myotubes

825 a) and b) Representative images of $\mathrm{Ca}^{2+}$ imaging in EDL and soleus-derived myotubes. Fura 2-AM was administered 
$827(0.9 \mathrm{~mm})$ for $1 \mathrm{~min}$ followed by a 1-min resting period in between. During the initial $0 \%$ stretch timepoint, Yoda1

828 cohorts were administered with $30 \mu \mathrm{M}$ of the agonist (red *) before being subjected to stretch. Ionomycin at $5 \mu \mathrm{M}$ was

829 then applied. Side vertical bar shows Fura 2-AM ratio emittance from low to high. c) and d) Average changes in the

830 intracellular $\mathrm{Ca}^{2+}$ level $\left(\left[\mathrm{Ca}^{2+}\right] \mathrm{i}\right.$,) difference between base and peak pixel value) normalised to ionomycin. Data is mean

$831 \pm$ SEM from three experiments $(n=3$ mice). *: Significant difference at $\mathrm{p}<0.05$ compared to control at each condition.

832 If: Significant difference at $p<0.05$ compared to $0 \%$ stretch counterparts. $\dagger$ : Significant difference at $p<0.05$

833 compared to 3\% stretch counterparts using one-way ANOVA followed by the Tukey-Kramer post-hoc. 
Ortuste Quiroga et al.

\section{Discussion}

The current study showed that the mechanosensitive ion channel Piezo1 is required for

the fusion and formation of myotubes. Indeed reduced expression of this channel hindered the

fusion of myocytes to forming or maturating myotubes - a phenotype that may be partly associated with interacting fusogenic proteins like Myomaker or Myomixer. We further showcased how agonist mediated activation of Piezo1 can enhance myogenic fusion at the expense of myotube width. Additionally, our findings revealed that over-activation of Piezo1

$\mathrm{Ca}^{2+}$ in response to stretch. Conversely, we showed how the downregulation of Piezol hinders $\mathrm{Ca}^{2+}$ entry into myotubes of both EDL and soleus muscle.

\section{Piezo1 in myogenesis}

The current study investigated the effects of Piezo1 regulation throughout the primary muscle cells derived from the mainly slow-type muscle soleus displayed higher expression of Piezol compared to the fast EDL muscle (Figure S1). Understanding the potential differences in muscle/fibre types and Piezol regulation is an intriguing area for future research and could reflect differences in the dynamics of myogenic progression. We also confirmed that the expression of Piezo2 is not altered by the down-regulation of Piezol. This is perhaps not surprising given the fact that Piezo2 is not as abundant in skeletal muscle compared to Piezol $[5,16]$. Nevertheless it was important to see any potential compensatory effects Piezo2 may impose. Specific downregulation of Piezol by siRNA-mediated 
Ortuste Quiroga et al.

860

861

862

863

864

865

866

867

868

869

870

871

872

873

874

875

876

877

879

880

881

882

883

derived primary myoblasts. However, our data do not exclude the possibility that Piezol is not involved in earlier myogenic events, perhaps in balancing quiescence and activation of satellite cells. In proliferating myoblasts, lack of Piezo1 function does not alter onset of myogenic differentiation, evaluated by the proportion of myogenin-positive cells. In contrast, where a significant phenotype was observed was in terminally differentiated myotubes. Indeed, our data found that knockdown of Piezol significantly reduced fusion of myocytes and prevented myotube formation and maturation. In contrast, activation of this $\mathrm{Ca}^{2+}$ permeable channel resulted in enhanced myogenic fusion. The main findings are summarised in Figure 9a-c.

\section{Piezo1 and muscular dystrophies}

Piezo1 activation showed a significant increase in the fusion index of both EDL- and soleus-derived myotubes. Although this phenotype could be viewed as beneficial in terms of muscular dystrophy prevention, we must be aware of the potential dangers of an overactive Piezo1 channel. In fact, we showed that even a 30 min incubation of myotubes with a high agonist concentration (100 $\mu \mathrm{M}$ of Yoda1 treatment) led to decreased fusion in both EDL- and soleus-derived myotubes (Figure 6). This adverse phenotype is most likely the result of a dangerously high $\mathrm{Ca}^{2+}$ influx. The publicly available data obtained from MRI-guided human biopsies from facioscapulohumeral muscular dystrophy (FSHD) affected individuals (Figure 9f and g) further highlights the necessity to examine mechanosensor channels like Piezo1 in muscular dystrophies. FSHD is associated with mutations in the distal end of chromosome 4 (4q35), resulting in epigenetic deregulation of the D4Z4 macrosatellite repeat array, causing uncontrolled expression of the transcription factor Double homeobox 4 (DUX4) [27-32]. In turn, DUX4 accumulation hampers myogenic progression promoting cytotoxicity and is 
Ortuste Quiroga et al.

considered the most possible root cause of FSHD pathogenesis [33]. We took advantage of a transcriptomic study on human DUX4-inducible myoblasts model elucidating DUX4's role in FSHD, to assess the level of PIEZO1 in such context and found that it is significantly downregulated upon accumulation of DUX4 [34] (Figure 9d), thus suggesting that PIEZO1 dysregulation may contribute to FSHD pathology.

In line with the muscular atrophy usually found in patients, human primary myoblasts derived from FSHD biopsies display reduced differentiation in vitro generating hypotrophic myotubes [35,36], thus resembling the effect of Piezol knockdown in our experimental setup. Indeed, analysis of transcriptomic dataset on differentiating isogenic FSHD affected or unaffected myoblasts previously performed by Banerji and colleagues [36], revealed that while during unaffected (healthy) myogenic differentiation, Piezol is upregulated from myoblasts to myotubes, its expression fails to increase in FSHD myogenic progression (Figure 9e). Moreover, our analyses of transcriptomic dataset from Wang and colleagues [37] found that PIEZO1 mRNA is significantly upregulated in MRI-guided muscle biopsy from FSHD patients that show the worst pathological phenotype (Group 4) (Figure 9f). Interestingly, it has recently been demonstrated that FSHD muscles display traces of ongoing regeneration, as indicated by elevated levels of embryonic Myosin, a canonical marker of ongoing muscle fibre regeneration [27]. Congruently with the accumulation of PIEZO1 mRNA, additional transcriptomic analysis also revealed a concomitant upregulation of MYOMAKER and MYOMIXER in the most affected group of muscle biopsies (Figure 9g) indicating an ongoing regeneration process. Such regeneration is further suggested by significant accumulation of embryonic MYH3 (myosin 3) mRNA (Figure 9g). In contrast, the reduced level of $M Y B P C 1$ (myosin binding protein $\mathrm{C} 1$ ), a myosin-associated protein involved in the formation and maintenance of sarcomere structure confirmed the dystrophic status of 
bioRxiv preprint doi: https://doi.org/10.1101/2020 09.27.315242; this version posted November 22,2020 . The copyright holder for this preprint (which was not certified by peer review) is the author/funder, who has granted bioRxiv a license to display the preprint in perpetuity. It is made available under aCC-BY-NC-ND 4.0 International license.

Ortuste Quiroga et al.

908

909

910

911

912

913

914

915

916

917

918

919

920

921

922

923

924

925

926

927

928

929

930

FSHD muscle biopsies (Figure 9g). Therefore, from these pieces of evidence we conclude that Piezol dysregulation may contribute to the pathomechanism underlying FSHD. However, the precise definition of PIEZOI dynamics in FSHD progression would require specific investigation.

a) Normal Muscle
Proliferation
Terminal differentiation
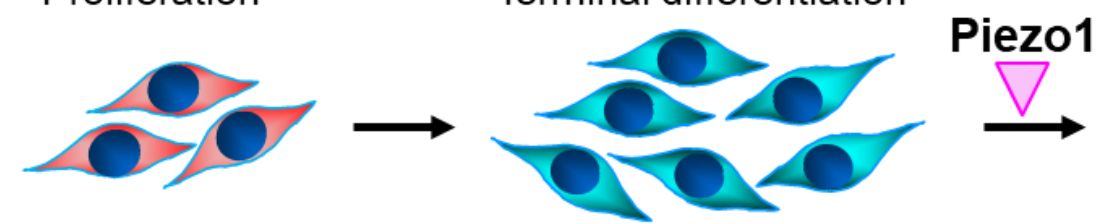

Fusion \& Growth

Myoblasts

Myocytes

Piezo1 Regulates Fusion \& Growth of Myofibres

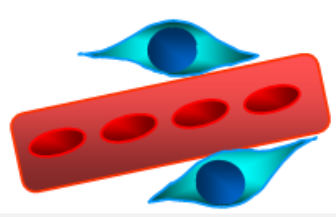

Myotubes

\section{b) Piezo1 Suppression}

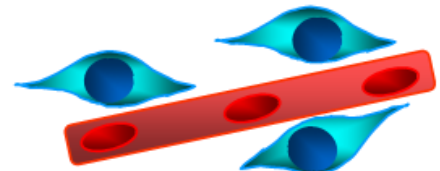

Piezo 1 Suppression Hinders Myotube Formation \& Growth e)
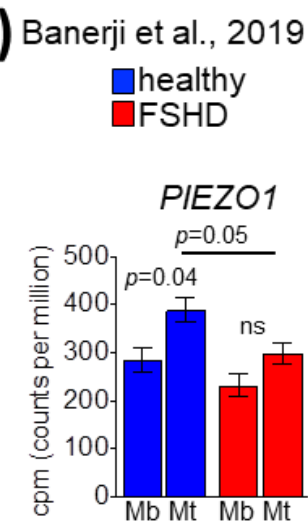

g) MYOMAKER

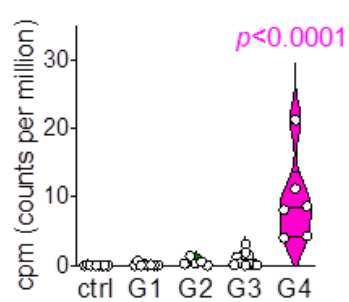

MYOMIXER

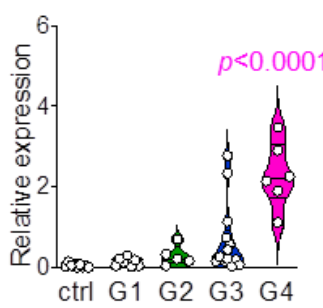

C) Piezo1 Activation

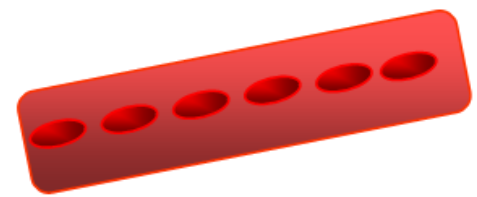

Piezo1 Activation Enhances Myotube Fusion \& Growth

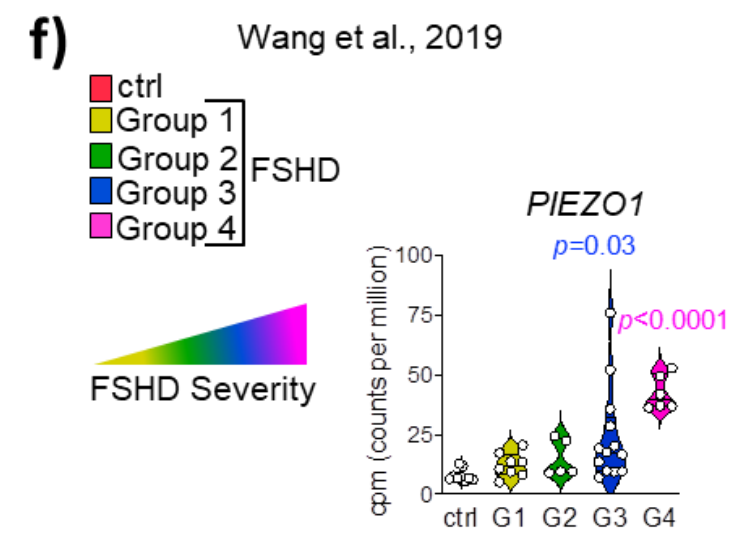

MYH3

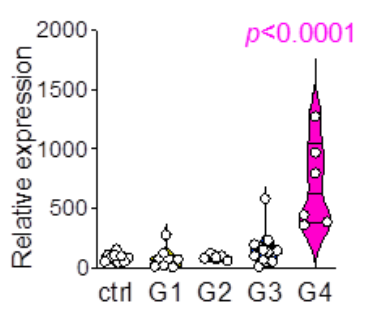

MYBPC1

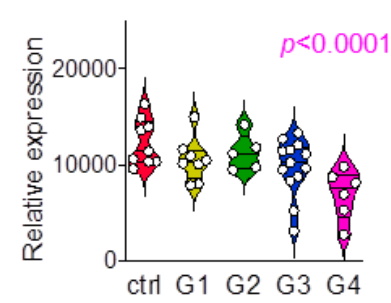


bioRxiv preprint doi: https://doi org/10.1101/2020.09.27.315242; this version posted November 22, 2020. The copyright holder for this preprint (which was not certified by peer review) is the author/funder, who has granted bioRxiv a license to display the preprint in perpetuity. It is made available under aCC-BY-NC-ND 4.0 International license.

Ortuste Quiroga et al.

Figure 9. Piezo1 which regulates myogenic fusion is dysregulated in FSHD muscle pathology.

933 a) Under normal conditions, membrane mechanosensors, such as Piezo1 (magenta arrowhead), regulate myocyte fusion thereby ensuring efficient muscle fibre formation and maturation. b) Suppression of Piezo1 expression blunts myocyte fusion and $\mathrm{Ca}^{2+}$ homeostasis, thus hampering the formation of proper myotubes. c) In contrast, selective chemical activation of Piezo1 function enhances myogenic fusion thus significantly increasing myotube formation and maturation. d) Statistical analysis derived from Choi et al, [34], shows that DUX4 expression induced by Doxycycline, which mimics human FSHD pathogenesis, results in significant suppression of PIEZOI level compared to control non-induced cells (Unpaired two-tailed t-test). e) Time-course transcriptomics analysis, derived from Banerji et al, [36], on differentiating isogenic (bar D4Z4 repeats) myoblasts derived from a male mosaic FSHD1 patient show that while unaffected (blue) myoblasts upregulated significantly PIEZOI expression when differentiating into myotubes, in FSHD PIEZO1 level remains unchanged from myoblasts to myotubes (red bar) (Unpaired two-tailed t-test). f) Statistical analysis of transcriptomic data on human FSHD biopsies derived from Wang et al [37]. Patients have been grouped according to FSHD severity, which increases from

944 Group1 to Group 4. Control (ctrl) represents non-affected individual. Count of gene reads (cpm, count per million) PIEZO1

945 is significantly upregulated in FSHD Group 4. g) The same dataset as (f) shows concomitant upregulation of MYOMAKER,

946 MYOMIXER and MYH3 (myosin 3) while reduced MYBPC1 (myosin binding protein C1) level in line with regeneration process ongoing and a severe dystrophic phenotype respectively. One-way ANOVA with Tukey's post-hoc test.

948

949

950

951

952 
Ortuste Quiroga et al.

The skeletal muscle has typically a slow turnover, thus putative fusion proteins like resistive exercise etc.). However in FSHD, the disorganised and possibly inefficient activation of the regeneration program may dysregulate Piezo1 expression. From a pathophysiological angle and taking into consideration our over-activation data using Yoda1 (Figure 6), we suggest that an over-abundance of Piezo1 expression in FSHD may result in improper Piezo1 expression may also lead to defective myogenic progression and muscle formation, as indicated by our knockdown analysis. In line with this, transcriptomic analysis of human myoblasts induced to express a high level of DUX4, which is considered to mimic some aspects of FSHD pathogenesis, revealed significant suppression of PIEZO1 expression pathogenesis.

\section{Piezo1 and the myocyte fusion machinery}

Piezol downregulation significantly reduced myocyte fusion during myotube formation and myotube maturation. To the best of our knowledge there is only one other paper published that examined Piezol in skeletal muscle by Tsuchiya et al. [17]. Interestingly, the findings from this group showed that Piezol inhibition resulted in a sheet-like syncytium of MyHC coupled with increased fusion. Although these findings show contrasting results to the ones presented in this study, we must take into consideration potential factors which may explain why this may be the case. One such factor is the method of Piezol inhibition used by

979 Tsuchiya et al, [17]. They carried out many of their experiments using knockout lines of 
Ortuste Quiroga et al.

express Piezol to begin with (unlike the cells we used) may yield completely different phenotypes, compared to the transient inhibition achieved by siRNA mediated transfection.

Therefore, complete lack of Piezol expression may favour the activation of a secondary, yet unknown, alternative Piezol-affected pathway(s) to fusion. Regarding the Piezol siRNA transfection experiments, although more than $60 \%$ reduction in gene expression is ideal; we nevertheless found that our level of Piezol knockdown produced very interesting effects on myogenic regulation. Moreover, Miyamoto et al, [16] also obtained slightly below or just about $60 \%$ Piezol reduction, yet reported intriguing Piezol-associated events in urothelial cells. Perhaps Tsuchiya et al, obtained even greater knockdown of Piezol in their siRNAmediated analyses [17], further suggesting that the timing and level of Piezol expression may yield varying phenotypes. There was also the likelihood that siRNA used in this study may potentially have off-target effects on other genes which could influence myotube formation now tested four different Piezol-specific siRNAs and all show a marked reduction in the fusion of myoblasts, compared to siRNA controls (Figure S4). Therefore, we conclude that impaired Piezo1 signalling inhibits myotube fusion. Further support comes from our Piezo1 activation experiments. Congruently, we found that selective activation of Piezo1 by the agonist Yoda1 significantly increased the fusion index in a concentration- and timedependent fashion. These results do not disprove the investigation by Tsuchiya et al, but rather highlight the complexity underlying Piezo1 dynamics. It would be interesting to see the effects of constitutive overexpression of Piezol by retroviral transduction or conditional Piezol transgenic mice. Furthermore, obtaining the cells used by Tsuchiya et al [17] and 
Ortuste Quiroga et al.

Additional support for the involvement of Piezo1 in myogenic fusion comes from our results which showed that its downregulation significantly reduced the expression of Myomaker - a muscle specific protein that localises to the plasma membrane and is crucial for vertebrate myocyte fusion [23,38,39]. Whether this is a direct response for the downregulation of Piezo1 or an indirect event remains to be determined. In addition, the expression level of Myomixer, another essential molecule for cell fusion, showed a tendency to reduce upon Piezol knockdown further suggesting that Piezol contributes in the regulation

1012

1013 of the cell fusion machinery. Removal of fusion proteins such as Myomaker results in decreased myoblast fusion, whereas overexpression results in enhanced fusion $[23,39,40]$.

A feature that became apparent in our study was the seemingly "thinner" myotubes in agonist treated samples which showed increased fusion. Indeed, compared to DMSO controls, the average myotube width was smaller in Yoda1 treated cohorts (Figure 7 and Figure S5).

Thus, we wonder whether the enhanced fusion, measured by the number of myonuclei within MyHC positive myotubes, comes at the expense of myotube syncytial maturation. In other words, Piezo1 activation may have hastened myonuclear fusion leading to premature myotube formation. Perhaps increased PIEZO1 expression (Figure 9f) contributes to the inadequate regenerative state of FSHD stricken muscle.

DMSO controls at the 4 hours mark showed a trending decrease in fusion in soleusderived myotubes, compared to DMSO counterparts from other timepoints. EDL-derived DMSO-treated myotubes at the 4-hour incubation timepoint, showed a statistically significant decline in fusion compared to DMSO counterparts. Although these differences may be negligible at lower timepoints, we should warrant concern over the potential for DMSOmediated inhibition of primary derived myotubes during longer timeframes. Indeed, the observed myotube inhibition may be slightly overestimated in Yoda1-treated samples at the 4-hour timepoint. 
Ortuste Quiroga et al.

\section{Piezol activation and $\mathrm{Ca}^{2+}$ influx}

The present study showed that selective downregulation of Piezol dramatically suppressed $\left[\mathrm{Ca}^{2+}\right] \mathrm{i}$, which most likely translates in the depression of the influx of $\mathrm{Ca}^{2+}$ into cultured myotubes exposed to stretch. In contrast we showed that activation of Piezo1 significantly increased $\left[\mathrm{Ca}^{2+}\right] \mathrm{i}$, which means the enhancement of $\mathrm{Ca}^{2+}$ influx. Our results propose that Piezo1 is a novel intracellular $\mathrm{Ca}^{2+}$ regulatory protein in skeletal muscle function. $\mathrm{Ca}^{2+}$ plays a crucial role in skeletal muscle function, maintenance and plasticity. All muscle fibres use $\mathrm{Ca}^{2+}$ as their main regulatory and signalling molecule [41-43]. Therefore, the contractile functionalities of muscle fibres are dependent on the highly regulated expression of proteins involved in $\mathrm{Ca}^{2+}$ handling and signalling. Our study showed that Piezo1 mediated regulation of $\mathrm{Ca}^{2+}$ influx is a key driving factor in the respective decrease and increase in myotube fusion in response to Piezo1 inhibition and activation. To the best of our knowledge this is the first time this has been demonstrated.

The silicon bio-chamber experiments revealed that at relatively low stretch conditions of 3\% $(0.3 \mathrm{~mm})$ neither EDL- nor soleus-derived myotubes elicited a significant increase in $\mathrm{Ca}^{2+}$ influx. However at higher stretch distances (6\% and 9\% stretch) this mechanical barrier was crossed as demonstrated by the net increase in $\left[\mathrm{Ca}^{2+}\right] \mathrm{i}$, compared to $0 \%$ stretch counterparts. In a similar set of experiments (albeit using urothelial cells), Miyamoto et al. also showed a distance dependent increase of $\mathrm{Ca}^{2+}$ influx. Interestingly this response was blunted in Piezol-siRNA-treated conditions [16]. The researchers also showed that a high enough $\left[\mathrm{Ca}^{2+}\right] \mathrm{i}$ must be attained in order to elicit a response, in their case ATP efflux. The data presented in this study supports the presence of a stretch-dependent increase in $\mathrm{Ca}^{2+}$ influx. Remarkably we found that activation of Piezo1 resulted in increased $\left[\mathrm{Ca}^{2+}\right] \mathrm{i}$ at $3 \%$ stretch conditions, suggesting that the activation threshold of Piezo1 was lowered. 
Ortuste Quiroga et al.

Furthermore the data showed that reduction of Piezol expression significantly blunted any significant increase of $\left[\mathrm{Ca}^{2+}\right] \mathrm{i}$ in response to stretch. These results, for the first time show the need for Piezo1 to respond to stretch and permeate $\mathrm{Ca}^{2+}$ into myotubes. The findings also propose the presence of a physical threshold that must be attained before Piezol mediated $\mathrm{Ca}^{2+}$ influx is significantly increased. Like Miyamoto et al [14], we find that a stretchdependent increase in $\mathrm{Ca}^{2+}$ influx is suppressed when Piezol expression is decreased. Conversely, we see an increase of $\left[\mathrm{Ca}^{2+}\right] \mathrm{i}$ when Piezol is activated. Whether this leads to altered cellular/myotube viability in the form of ATP release remains a subject for future research.

\section{Conclusion}

The data presented in this study showed that the Piezo1 channel is present in primary derived myoblasts and myotubes but expressed at a higher proportion in the latter. Downregulation of Piezol significantly lowered the fusion capacity during myotube formation and maturation. In contrast, Piezo1 activation increased fusion. Future research examining changes in myotube function (integrity, $\mathrm{Ca}^{2+}$ influx, cytoskeletal organisation and fusion) that are directly the result of mechanical stress should consider analysis of Piezo1. In the context of therapeutic strategies against muscular dystrophies such as FSHD, not only must we unravel the spatiotemporal regulation of Piezol expression, but we must be aware of this channel's ability to alter it $\mathrm{Ca}^{2+}$ influx threshold by adapting or inactivating its gating capacity in response to repetitive stimuli. Pharmaceutically, small activating molecules such as Yoda1 (and others like it) may prove beneficial. However careful attention must be given to the half-life and pharmacokinetics of these agonists in vivo before even considering them as viable drugs for human consumption. Piezol's importance in skeletal muscle maintenance 
bioRxiv preprint doi: https://doi org/10.1101/2020.09.27.315242; this version posted November 22, 2020. The copyright holder for this preprint (which was not certified by peer review) is the author/funder, who has granted bioRxiv a license to display the preprint in perpetuity. It is made available under aCC-BY-NC-ND 4.0 International license.

Ortuste Quiroga et al.

1078

and function will undoubtedly grow as new research aims to explore the mechanisms and

1079 signalling pathways this remarkable mechanosensor employs.

1080

1081 
Ortuste Quiroga et al.

\section{Funding}

1083 This work was supported by KAKENHI (JP17K01762， K.G.; JP18H03160, K.G.; 1084 JP19K22825, K.G.; 19KK0254. K.G.) from the Japan Society for the Promotion of Science, 1085 the Science Research Promotion Fund from the Promotion and Mutual Aid Corporation for 1086 Private Schools of Japan, and Graduate School of Health Sciences, Toyohashi SOZO 1087 University (K.G.).

1088

1089 Acknowledgments

1090 The authors thank Ms. Yumiko Asakura and from Department of Physiology, Graduate 1091 School of Health Sciences, Toyohashi SOZO University for her assistance.

\section{Conflicts of Interest}

The authors declare that there are no conflicts of interest. 
Ortuste Quiroga et al.

\section{References}

1097

1. Mauro A (1961) Satellite cell of skeletal muscle fibers. The Journal of biophysical and biochemical cytology 9:493-495

1099

2. Zammit PS, Relaix F, Nagata Y, Ruiz AP, Collins CA, Partridge TA, Beauchamp JR 1100 (2006) Pax7 and myogenic progression in skeletal muscle satellite cells. Journal of cell science 119 (Pt 9):1824-1832

1102

3. Zammit PS, Partridge TA, Yablonka-Reuveni Z (2006) The skeletal muscle satellite cell: the stem cell that came in from the cold. J Histochem Cytochem 54:1177-1191

4. Relaix F, Zammit PS (2012) Satellite cells are essential for skeletal muscle regeneration: the cell on the edge returns centre stage. Development (Cambridge, England) 139 (16):28452856

1107

5. Coste B, Mathur J, Schmidt M, Earley TJ, Ranade S, Petrus MJ, Dubin AE, Patapoutian A (2010) Piezo1 and Piezo2 are essential components of distinct mechanically activated cation

1109 channels. Science (New York, NY) 330 (6000):55-60

6. Wang Y, Chi S, Guo H, Li G, Wang L, Zhao Q, Rao Y, Zu L, He W, Xiao B (2018) A lever-like transduction pathway for long-distance chemical- and mechano-gating of the mechanosensitive Piezo1 channel. Nature communications 9 (1):1300-1300 mechanotransductive release of ATP from human RBCs. Proc Natl Acad Sci U S A 112 1115 (38):11783-11788

1117 Architecture of the mammalian mechanosensitive Piezo1 channel. Nature 527:64 
Ortuste Quiroga et al.

1120

1121

1122

1123

1124

1125

1126

1127

1128

1129

1130

1131

1132

1133

1134

1135

1136

1137

1138

1139

1140

1141

1142

1143

1144

10. Saotome K, Murthy SE, Kefauver JM, Whitwam T, Patapoutian A, Ward AB (2017)

Structure of the mechanically activated ion channel Piezo1. Nature 554:481

11. Zhao Q, Zhou H, Li X, Xiao B (2018) The mechanosensitive Piezo1 channel: a threebladed propeller-like structure and a lever-like mechanogating mechanism. The FEBS journal 12. Zhao Q, Zhou H, Chi S, Wang Y, Wang J, Geng J, Wu K, Liu W, Zhang T, Dong M-Q, Wang J, Li X, Xiao B (2018) Structure and mechanogating mechanism of the Piezo1 channel. Nature 554:487

13. Coste B, Murthy SE, Mathur J, Schmidt M, Mechioukhi Y, Delmas P, Patapoutian A (2015) Piezo1 ion channel pore properties are dictated by C-terminal region. Nature Communications 6 (1):7223

14. Franco-Obregón A, Lansman J (1990) Calcium entry through stretch-in-activated ion channels in MDX myotubes. Nature 344:670-673

15. Mercuri E, Bönnemann CG, Muntoni F (2019) Muscular dystrophies. Lancet 394 (10213):2025-2038

16. Miyamoto T, Mochizuki T, Nakagomi H, Kira S, Watanabe M, Takayama Y, Suzuki Y, Koizumi S, Takeda M, Tominaga M (2014) Functional role for Piezo1 in stretch-evoked $\mathrm{Ca}(2)(+)$ influx and ATP release in urothelial cell cultures. J Biol Chem 289 (23):1656516575

17. Tsuchiya M, Hara Y, Okuda M, Itoh K, Nishioka R, Shiomi A, Nagao K, Mori M, Mori Y, Ikenouchi J, Suzuki R, Tanaka M, Ohwada T, Aoki J, Kanagawa M, Toda T, Nagata Y, Matsuda R, Takayama Y, Tominaga M, Umeda M (2018) Cell surface flip-flop of phosphatidylserine is critical for PIEZO1-mediated myotube formation. Nature Communications 9 (1):2049

18. Collins CA, Zammit PS (2009) Isolation and grafting of single muscle fibres. Methods in molecular biology (Clifton, NJ) 482:319-330 
bioRxiv preprint doi: https://doi.org/10.1101/2020.09.27.315242; this version posted November 22, 2020. The copyright holder for this preprint (which was not certified by peer review) is the author/funder, who has granted bioRxiv a license to display the preprint in perpetuity. It is made available under aCC-BY-NC-ND 4.0 International license.

Ortuste Quiroga et al.

19. Lacroix JJ, Botello-Smith WM, Luo Y (2018) Probing the gating mechanism of the mechanosensitive channel Piezo1 with the small molecule Yoda1. Nature Communications 9 (1):2029

20. Nourse JL, Pathak MM (2017) How cells channel their stress: Interplay between Piezo1 and the cytoskeleton. Seminars in cell \& developmental biology 71:3-12

21. McHugh BJ, Murdoch A, Haslett C, Sethi T (2012) Loss of the integrin-activating transmembrane protein Fam38A (Piezo1) promotes a switch to a reduced integrin-dependent mode of cell migration. PLoS One 7 (7):e40346-e40346

22. Schnorrer F, Dickson BJ (2004) Muscle building; mechanisms of myotube guidance and attachment site selection. Dev Cell 7 (1):9-20

23. Millay DP, O'Rourke JR, Sutherland LB, Bezprozvannaya S, Shelton JM, Bassel-Duby R, Olson EN (2013) Myomaker is a membrane activator of myoblast fusion and muscle formation. Nature 499 (7458):301-305

24. Bi P, Ramirez-Martinez A, Li H, Cannavino J (2017) Control of muscle formation by the fusogenic micropeptide myomixer. 356 (6335):323-327

25. Quinn ME, Goh Q, Kurosaka M, Gamage DG, Petrany MJ, Prasad V, Millay DP (2017) Myomerger induces fusion of non-fusogenic cells and is required for skeletal muscle development. Nature Communications 8 (1):15665

26. Zhang Q, Vashisht AA, O’Rourke J, Corbel SY, Moran R, Romero A, Miraglia L, Zhang J, Durrant E, Schmedt C, Sampath SC, Sampath SC (2017) The microprotein Minion controls cell fusion and muscle formation. Nature Communications 8 (1):15664

27. Banerji CRS, Henderson D, Tawil RN, Zammit PS (2020) Skeletal muscle regeneration in facioscapulohumeral muscular dystrophy is correlated with pathological severity. Human Molecular Genetics 
Ortuste Quiroga et al.

28. Bosnakovski D, Xu Z, Gang EJ, Galindo CL, Liu M, Simsek T, Garner HR, AghaMohammadi S, Tassin A, Coppee F, Belayew A, Perlingeiro RR, Kyba M (2008) An isogenetic myoblast expression screen identifies DUX4-mediated FSHD-associated molecular pathologies. EMBO J 27

29. Deidda G, Cacurri S, Piazzo N, Felicetti L (1996) Direct detection of 4q35 rearrangements implicated in facioscapulohumeral muscular dystrophy (FSHD). J Med Genet 33

30. Ferreboeuf M, Mariot V, Bessieres B, Vasiljevic A, Attie-Bitach T, Collardeau S, Morere J, Roche S, Magdinier F, Robin-Ducellier J, Rameau P, Whalen S, Desnuelle C, Sacconi S, Mouly V, Butler-Browne G, Dumonceaux J (2014) DUX4 and DUX4 downstream target genes are expressed in fetal FSHD muscles. Hum Mol Genet 23

31. Tassin A, Laoudj-Chenivesse D, Vanderplanck C, Barro M, Charron S, Ansseau E, Chen YW, Mercier J, Coppee F, Belayew A (2013) DUX4 expression in FSHD muscle cells: how could such a rare protein cause a myopathy? J Cell Mol Med 17

32. Tawil R, van der Maarel SM, Tapscott SJ (2014) Facioscapulohumeral dystrophy: the path to consensus on pathophysiology. Skeletal Muscle 4 (1):12

33. Himeda CL, Jones PL (2019) The Genetics and Epigenetics of Facioscapulohumeral Muscular Dystrophy. Annual Review of Genomics and Human Genetics 20 (1):265-291

34. Choi SH, Gearhart MD (2016) DUX4 recruits p300/CBP through its C-terminus and induces global H3K27 acetylation changes. 44 (11):5161-5173

35. Barro M, Carnac G, Flavier S, Mercier J, Vassetzky Y, Laoudj-Chenivesse D (2010) Myoblasts from affected and non-affected FSHD muscles exhibit morphological differentiation defects. Journal of cellular and molecular medicine 14 (1-2):275-289

36. Banerji CRS, Panamarova M, Pruller J, Figeac N, Hebaishi H, Fidanis E, Saxena A, Contet J, Sacconi S, Severini S, Zammit PS (2019) Dynamic transcriptomic analysis reveals 
Ortuste Quiroga et al.

suppression of PGC $1 \alpha / E R R \alpha$ drives perturbed myogenesis in facioscapulohumeral muscular dystrophy. Hum Mol Genet 28 (8):1244-1259

37. Wang LH, Friedman SD, Shaw D, Snider L, Wong C-J, Budech CB, Poliachik SL, Gove NE, Lewis LM, Campbell AE, Lemmers RJFL, Maarel SM, Tapscott SJ, Tawil RN (2018) MRI-informed muscle biopsies correlate MRI with pathology and DUX4 target gene expression in FSHD. Human Molecular Genetics 28 (3):476-486

38. Ganassi M, Badodi S (2018) Myogenin promotes myocyte fusion to balance fibre number and size. $9(1): 4232$

39. Shi J, Cai M, Si Y, Zhang J, Du S (2018) Knockout of myomaker results in defective myoblast fusion, reduced muscle growth and increased adipocyte infiltration in zebrafish skeletal muscle. Human Molecular Genetics 27 (20):3542-3554

40. Goh Q, Millay DP (2017) Requirement of myomaker-mediated stem cell fusion for skeletal muscle hypertrophy. 6

41. Berchtold MW, Brinkmeier H, Muntener M (2000) Calcium ion in skeletal muscle: its crucial role for muscle function, plasticity, and disease. Physiological reviews 80 (3):12151265

42. Gehlert S, Bloch W, Suhr F (2015) Ca2+-dependent regulations and signaling in skeletal muscle: from electro-mechanical coupling to adaptation. Int J Mol Sci 16 (1):1066-1095 43. Allen DG, Lamb GD, Westerblad H (2008) Skeletal muscle fatigue: cellular mechanisms. Physiological reviews 88 (1):287-332 
Ortuste Quiroga et al.

1221

1222

1223

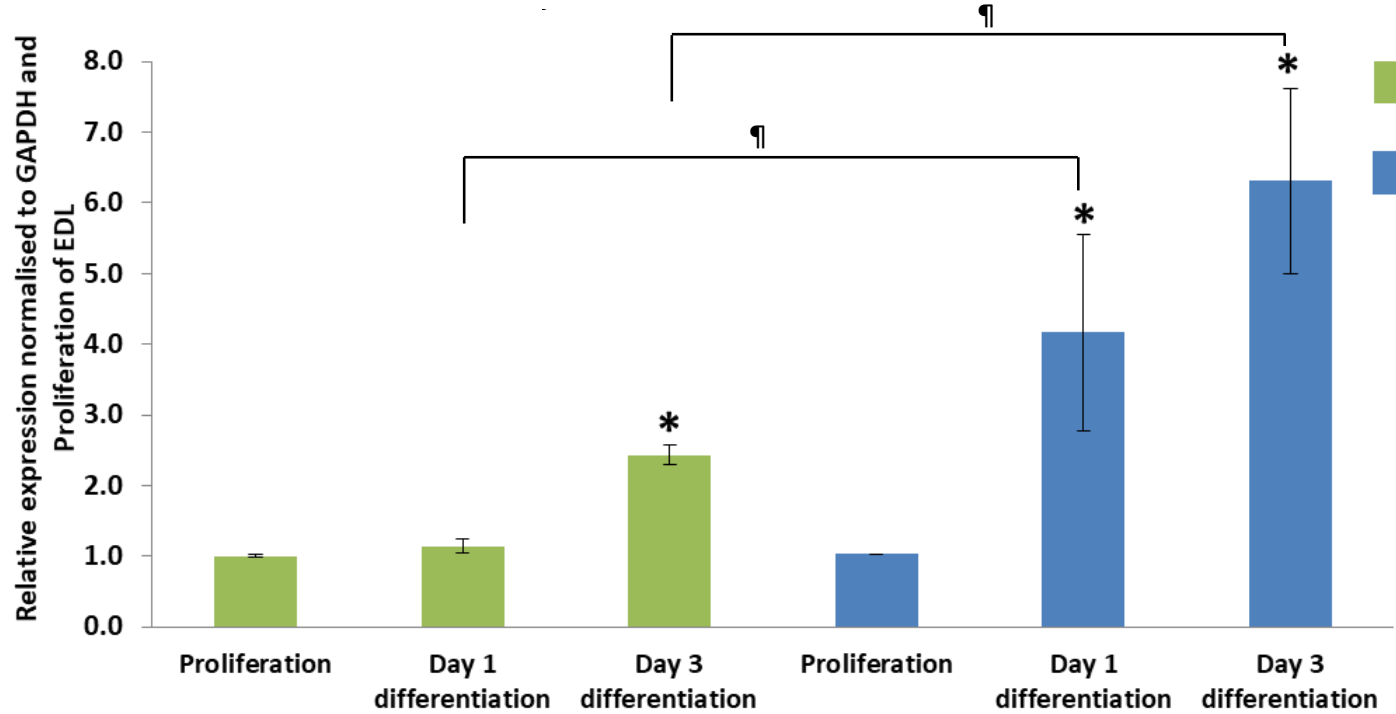

EDL

Soleus

Supplementary figure 1. Piezo1 is expressed at a higher level in soleus compared to EDL muscle-derived myoblasts.

Relative fold changes in expression of Piezol in myoblasts from EDL (green bars) and Soleus muscle (blue bars), during proliferation and through differentiation; 24 hours (Day 1) and 72 hours (Day 3) in differentiation medium. Values were normalised to Gapdh and then expressed as fold change compared to levels of proliferation in EDL samples. Data is presented as mean \pm SEM from three experiments $(\mathrm{n}=3$ mice). Asterisks $(*)$ denote significance at $\mathrm{p}<0.05$ compared to control proliferation conditions. II: Significant difference at $\mathrm{p}<0.05$ significant compared to either day 1 or day 3 differentiation cohorts using one-way ANOVA followed by the Tukey-Kramer post-hoc. 
bioRxiv preprint doi: https://doi.org/10.1101/2020.09.27.315242; this version posted November 22, 2020. The copyright holder for this preprint (which was not certified by peer review) is the author/funder, who has granted bioRxiv a license to display the preprint in perpetuity. It is made available under aCC-BY-NC-ND 4.0 International license.

Ortuste Quiroga et al.

a)

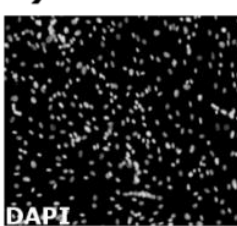

SiRNA control in EDL
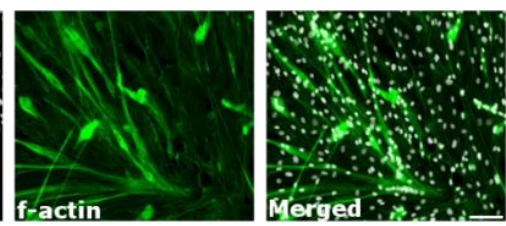

SiRNA Piezo1 in EDL
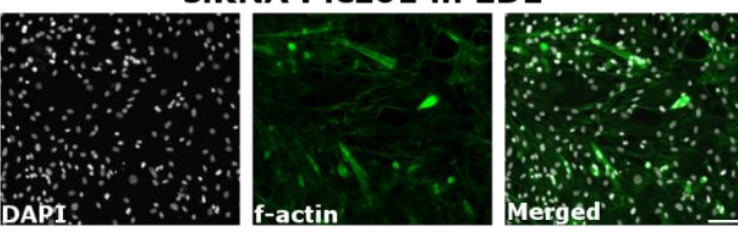

1240

1241

1242

1243

1244

1245

1246

1247

1248

1249

1250

1251

1254

1255

1256

1257

1258

1259

1260

1261 c)

Flouresence intensisty in EDL muscle

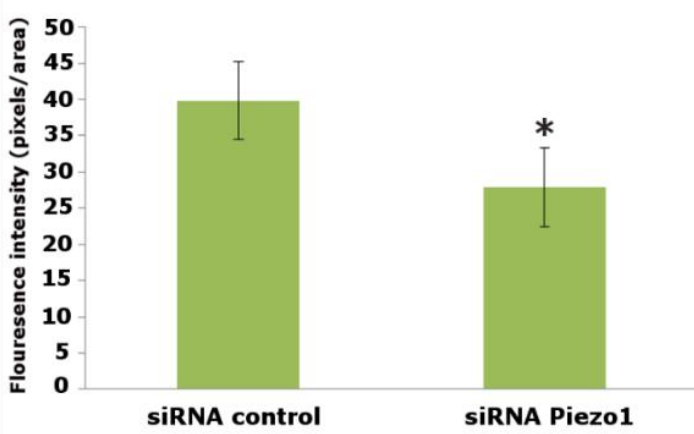

e)

Proportion of Phalloidin intensity in EDL muscle

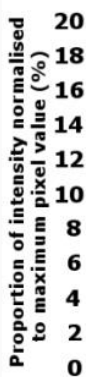

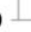

SiRNA control

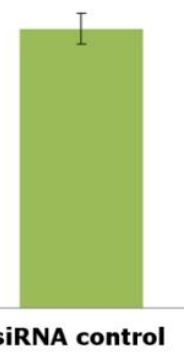

SiRnA Piezo1

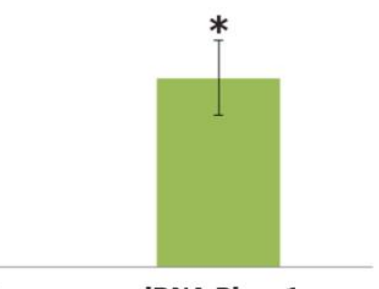

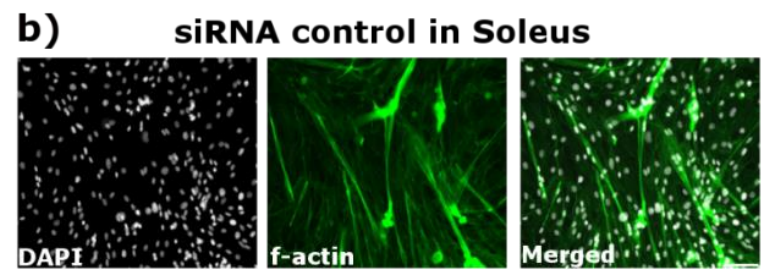

SiRNA Piezo1 in Soleus
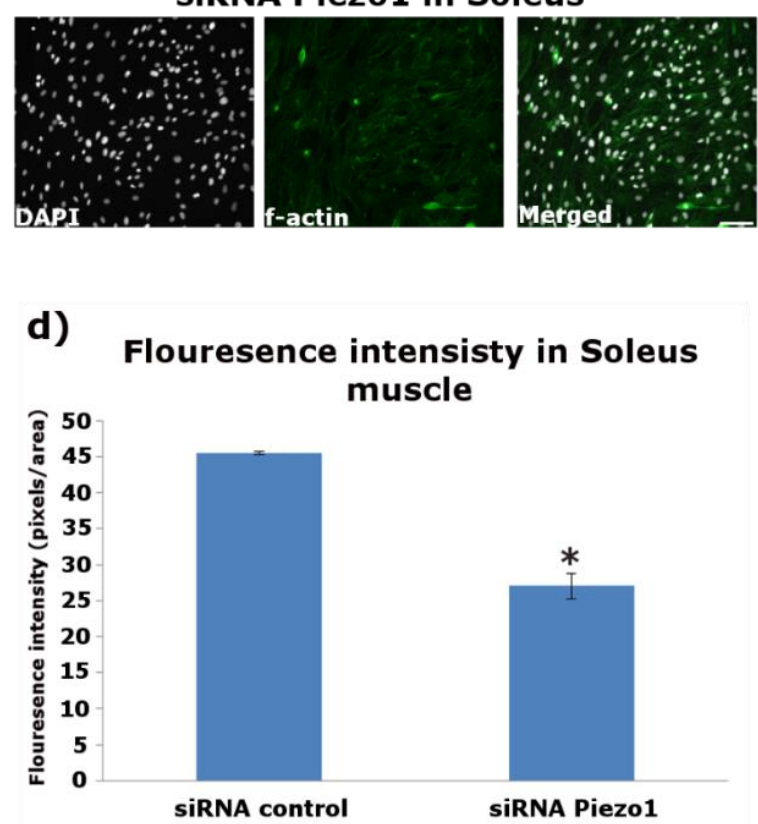

f)

Proportion of Phalloidin intensity in Soleus muscle

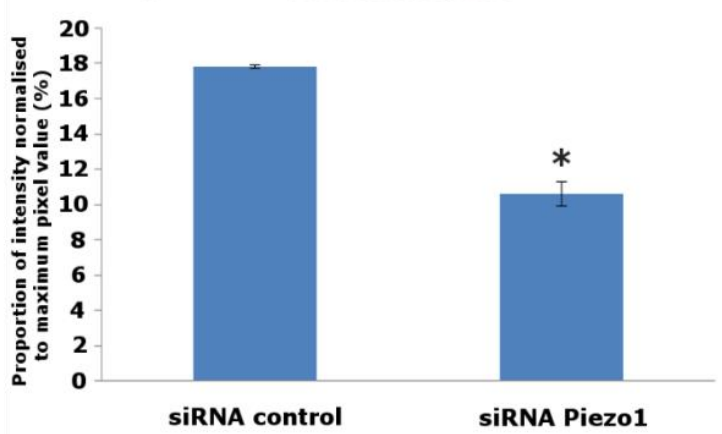

Supplementary Figure 2. Piezo1 knockdown reduced f-actin intensity in EDL and soleus-derived myotubes

a) and b) Representative images of EDL and soleus muscle--derived myotubes. Early forming myotubes were transfected with 10nM of control-siRNA (siScrambled) or Piezol-siRNA. Following overnight incubation, cells incubated for a further 72 hours. The cytoskeleton protein f-actin was visualised using fluorescently labelled phalloidin (green panels). Nuclei were counterstained with DAPI (black and white panels). Scale bar is $100 \mu \mathrm{m} . \mathrm{c}$ ) and d) Overall fluorescence intensity measured by pixel/area in each field of view (six images per conditions). e) and f) is data from c) and d) expressed as percentages relative to the maximum pixel value. Data is mean \pm SEM from three experiments $(n=$ 3 mice). *: Significant at $\mathrm{p}<0.05$ compared to siScrambled conditions using a 2-tailed paired student t-test. 
bioRxiv preprint doi: https://doi org/10.1101/2020.09 27 315242 - this version posted November 22, 2020. The copyright holder for this preprint (which was not certified by peer review) is the author/funder, who has granted bioRxiv a license to display the preprint in perpetuity. It is made available under aCC-BY-NC-ND 4.0 International license.

Ortuste Quiroga et al.

1262

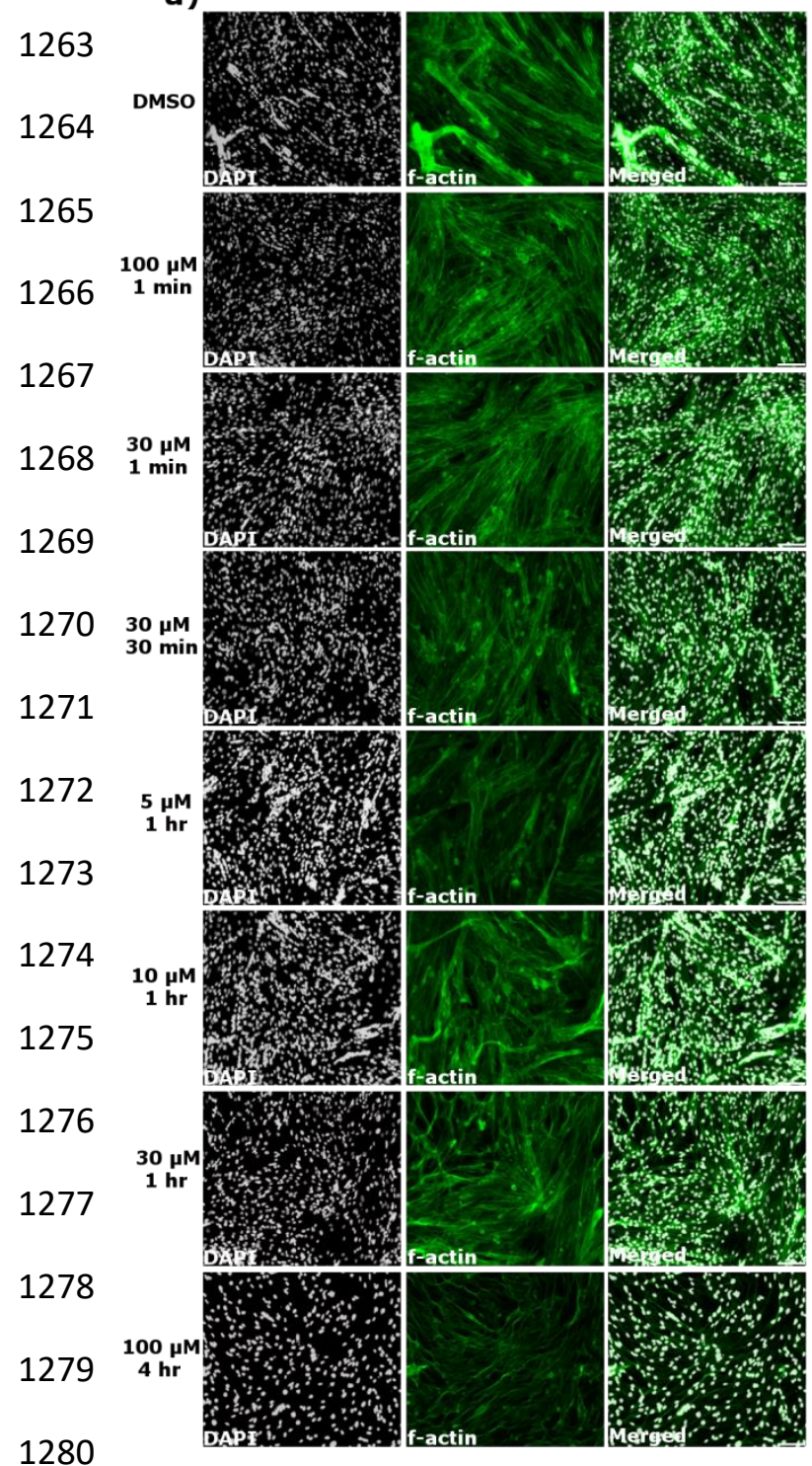

1281 b)

b) Proportion of phalloidin

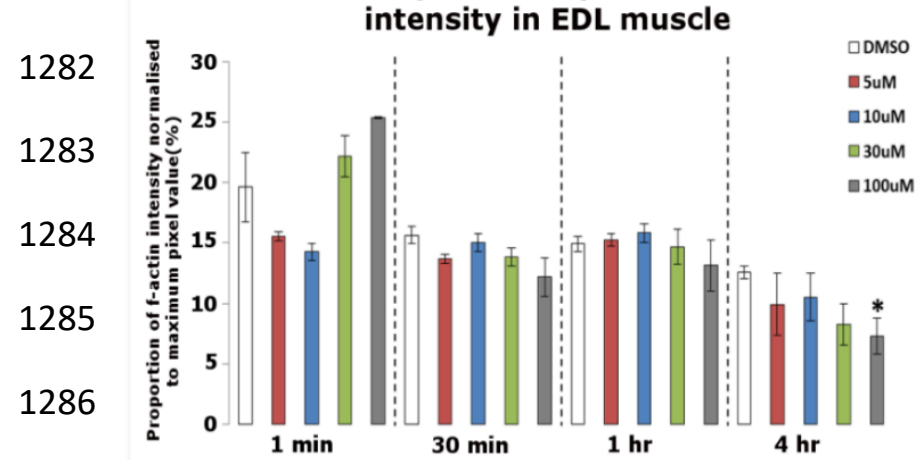

c)

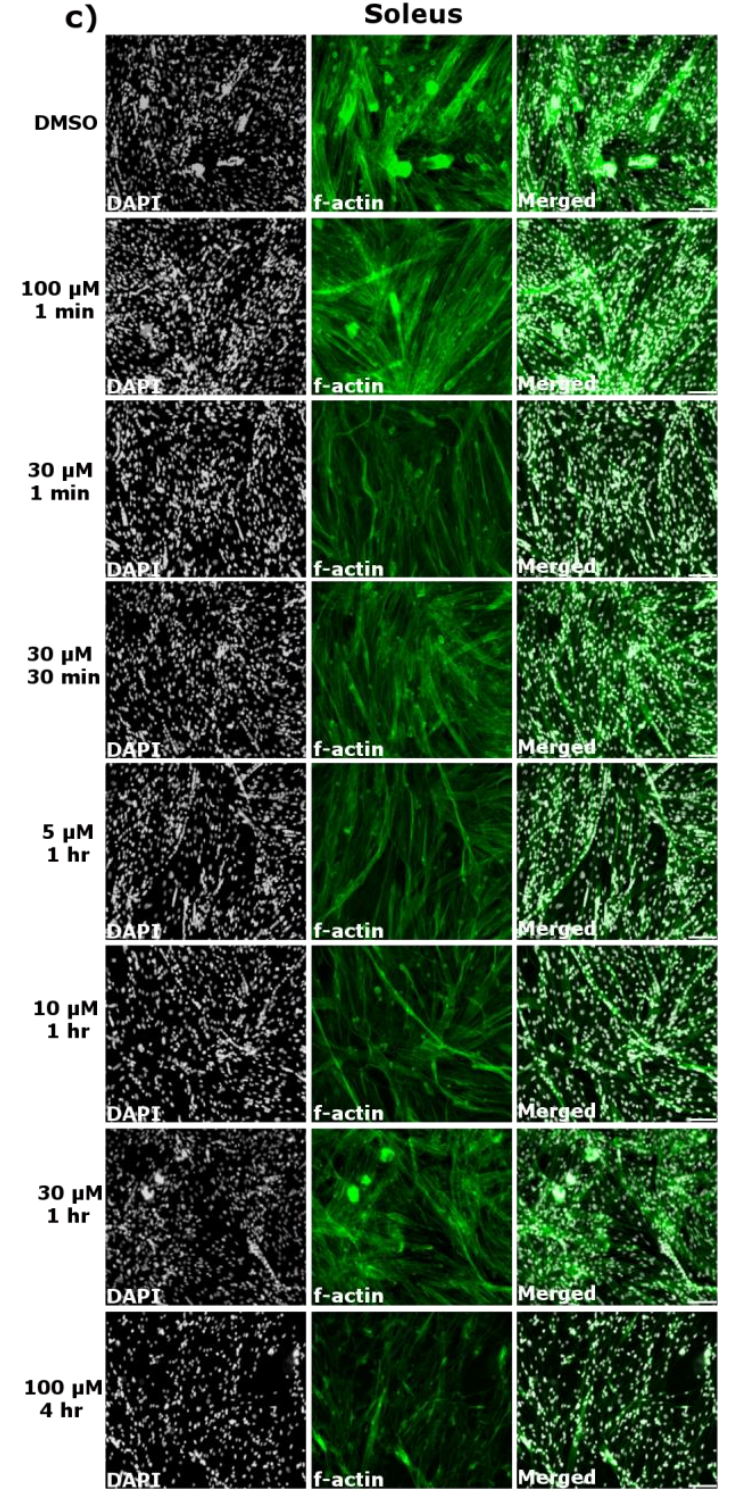

d)
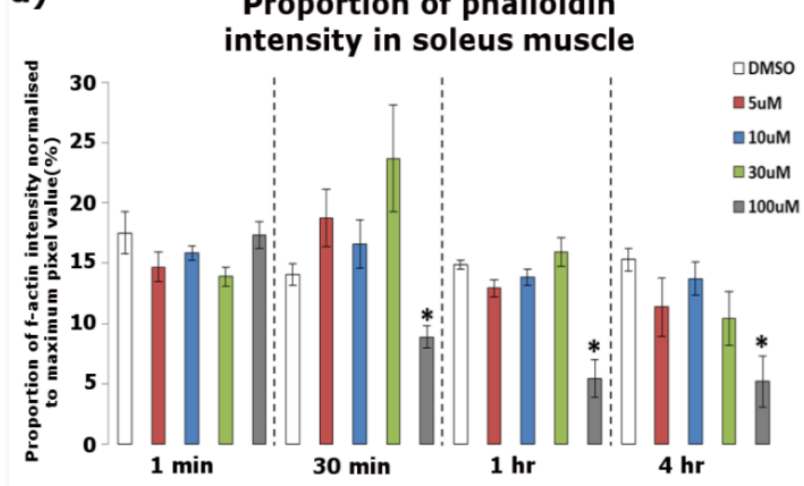

1287 
bioRxiv preprint doi: https://doi org/10.1101/2020.09.27.315242; this version posted November 22, 2020. The copyright holder for this preprint (which was not certified by peer review) is the author/funder, who has granted bioRxiv a license to display the preprint in perpetuity. It is made available under aCC-BY-NC-ND 4.0 International license.

Ortuste Quiroga et al.

Supplementary figure 3. Yoda1-mediated activation of Piezo1 does not increase f-actin intensity

1289 Early forming myotubes were administered with either DMSO (control, white bars) or Yoda1 at the following concentration: $5 \mu \mathrm{M}$ (red bars), $10 \mu \mathrm{M}$ (blue bars), $30 \mu \mathrm{M}$ (green bars) and $100 \mu \mathrm{M}$ (grey bars). Myotubes were incubated for $1 \mathrm{~min}, 10 \mathrm{~min}, 30 \mathrm{~min}, 1$ hour and 4 hours. Following the incubation period, the medium was exchanged with fresh reduced medium (without agonist) and myotubes were incubated for a further 2 days. b) and d) Representative images at relevant timepoints and concentrations treated with fluorescently labelled phalloidin (green panels and counterstained with DAPI (black and white panels). Images taken at x20 magnification. Scale bar is $100 \mu \mathrm{m}$. Bar graphs display proportion of f-actin intensity in a) EDL and b) soleus derived myotubes from each time point and concentration variables. Values are mean \pm SEM. * indicates significance at $\mathrm{p}<0.05$ using one-way ANOVA tests followed by the Tukey-Kramer post-hoc. $\mathrm{n}=3$ mice. 
bioRxiv preprint doi: https://doi.org/10.1101/2020.0927 315242. this version posted November 22, 2020. The copyright holder for this preprint (which was not certified by peer review) is the author/funder, who has granted bioRxiv a license to display the preprint in perpetuity. It is made available under aCC-BY-NC-ND 4.0 International license.

Ortuste Quiroga et al.

a)

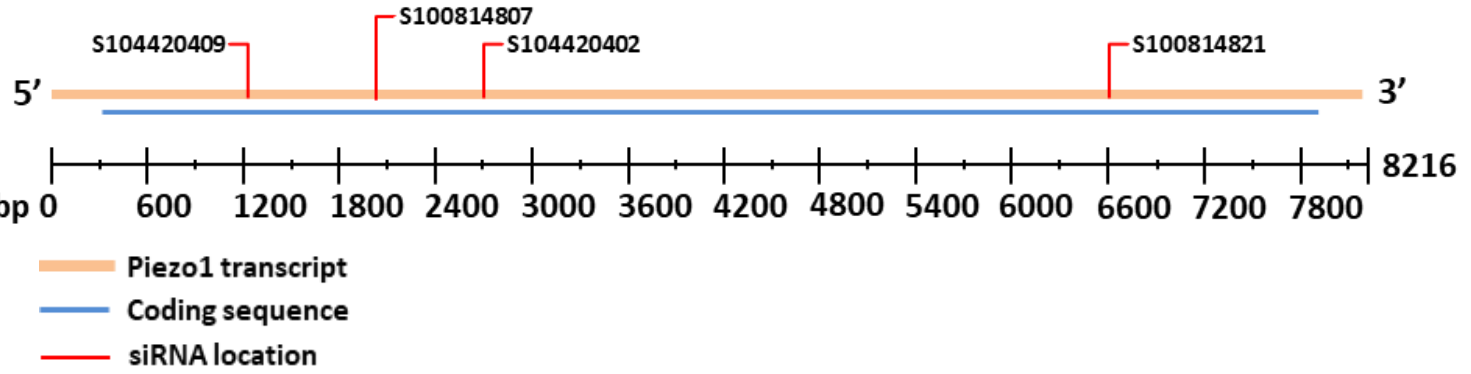

1303

1304

1305

1306

b)

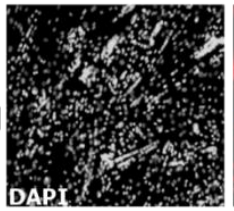

Piezo1 SiRNA 1
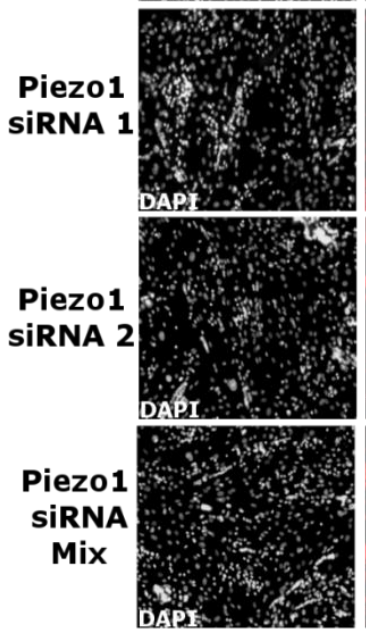

EDL
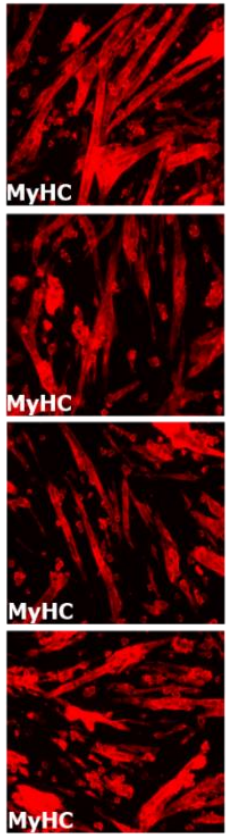
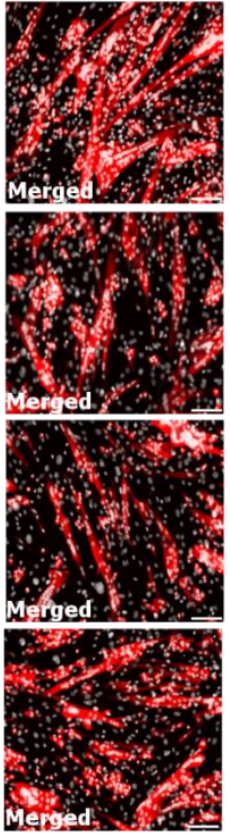
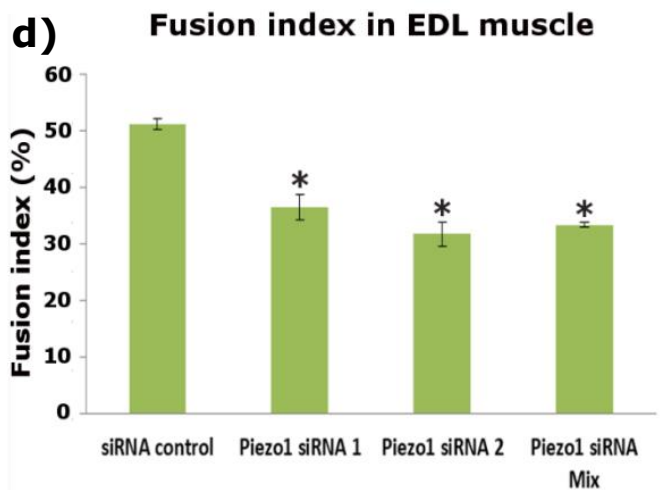

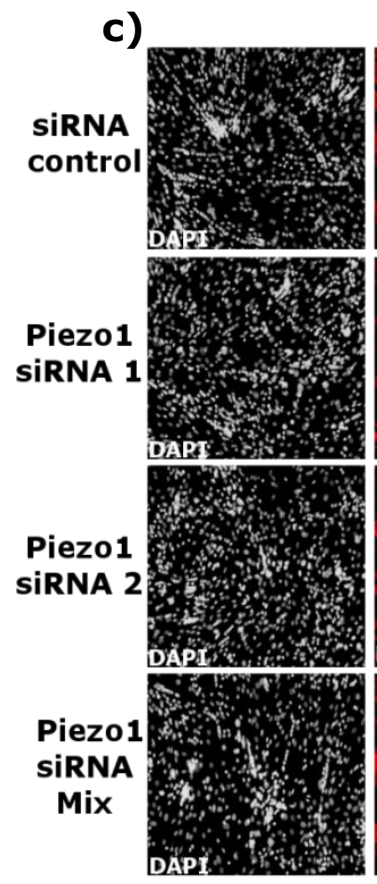

Soleus

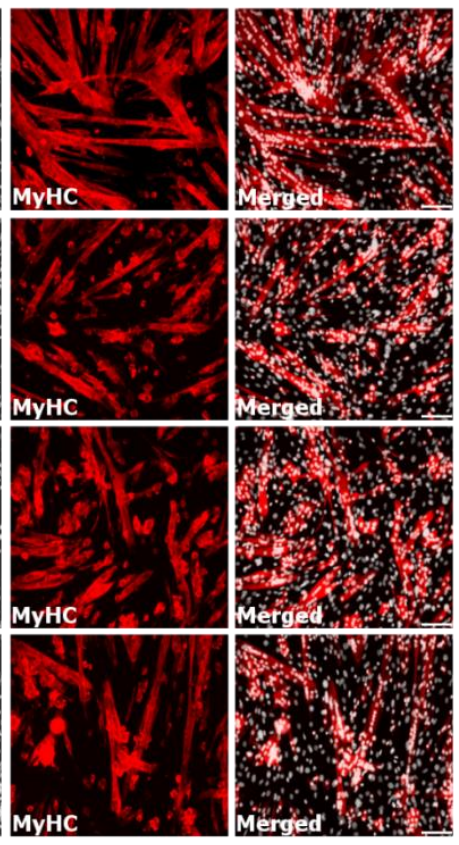

e) Fusion index in Soleus muscle

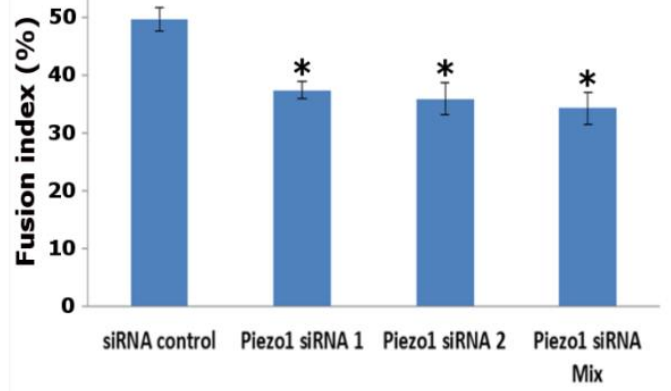

Supplementary figure 4. Different Piezo1 siRNA targets continue to show decrease in myotube fusion

a) Location of siRNAs shown in Table 1 on Piezol mRNA. Diagram shows Piezol mRNA transcript (orange bar), coding sequence (blue bar) and location of each siRNA (red line). bp: base pair. Diagram is adapted from Qiagen (https://geneglobe.qiagen.com/product-groups/flexitube-sirna). b and c) Representative images of EDL and soleus 
bioRxiv preprint doi: https://doi.org/10.1101/2020.09.27.315242; this version posted November 22, 2020. The copyright holder for this preprint (which was not certified by peer review) is the author/funder, who has granted bioRxiv a license to display the preprint in perpetuity. It is made available under aCC-BY-NC-ND 4.0 International license.

Ortuste Quiroga et al.

1325 muscle-derived myotubes, transfected with 10nM of control-siRNA (siScrambled) or different siRNAs specific for

1326 Piezol (Piezol-siRNA 1 and Piezol-siRNA 2). A mixture of four different Piezol-siRNAs (Piezol-siRNA Mix)

1327 including the one in the main text (Table 2) was also used at 10nM (2.5 nm each). Myoblasts were transfected and

1328 incubated overnight; cells were incubated for a further 72 hours. Cells were immunolabelled for Myosin heavy chain

1329 (MyHC) (red panels) and counterstained with DAPI (black and white panels). d) and e) The fusion index was

1330 calculated by counting the total number of nuclei within each myofibre and representing this as a percentage relative to

1331 the total number nuclei in the image taken. Data is mean \pm SEM from three experiments $(n=3$ mice $)$ *: Significant at

$1332 \mathrm{p}<0.05$ compared to siScrambled conditions using one-way ANOVA followed by the Tukey-Kramer post-hoc. From

1333 table 2, Piezo1 siRNA 1 corresponds to Qiagen, S104420402. Piezo1 siRNA 2 corresponds to Qiagen, S100814807. 
bioRxiv preprint doi: https://doi.org/10.1101/2020.09.27.315242; this version posted November 22, 2020. The copyright holder for this preprint (which was not certified by peer review) is the author/funder, who has granted bioRxiv a license to display the preprint in perpetuity. It is made available under aCC-BY-NC-ND 4.0 International license.

Ortuste Quiroga et al.

1335

1336

1337

1338

1339

1340

1341

1345

1346

1347

1348

1349

1350

1351

1352

1353

1354

1355

1356

1357

1358

1359

1360

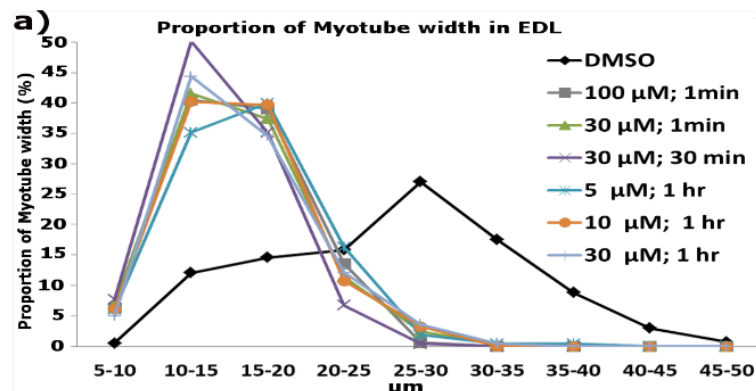

b) Proportion of Myotube width in Soleus
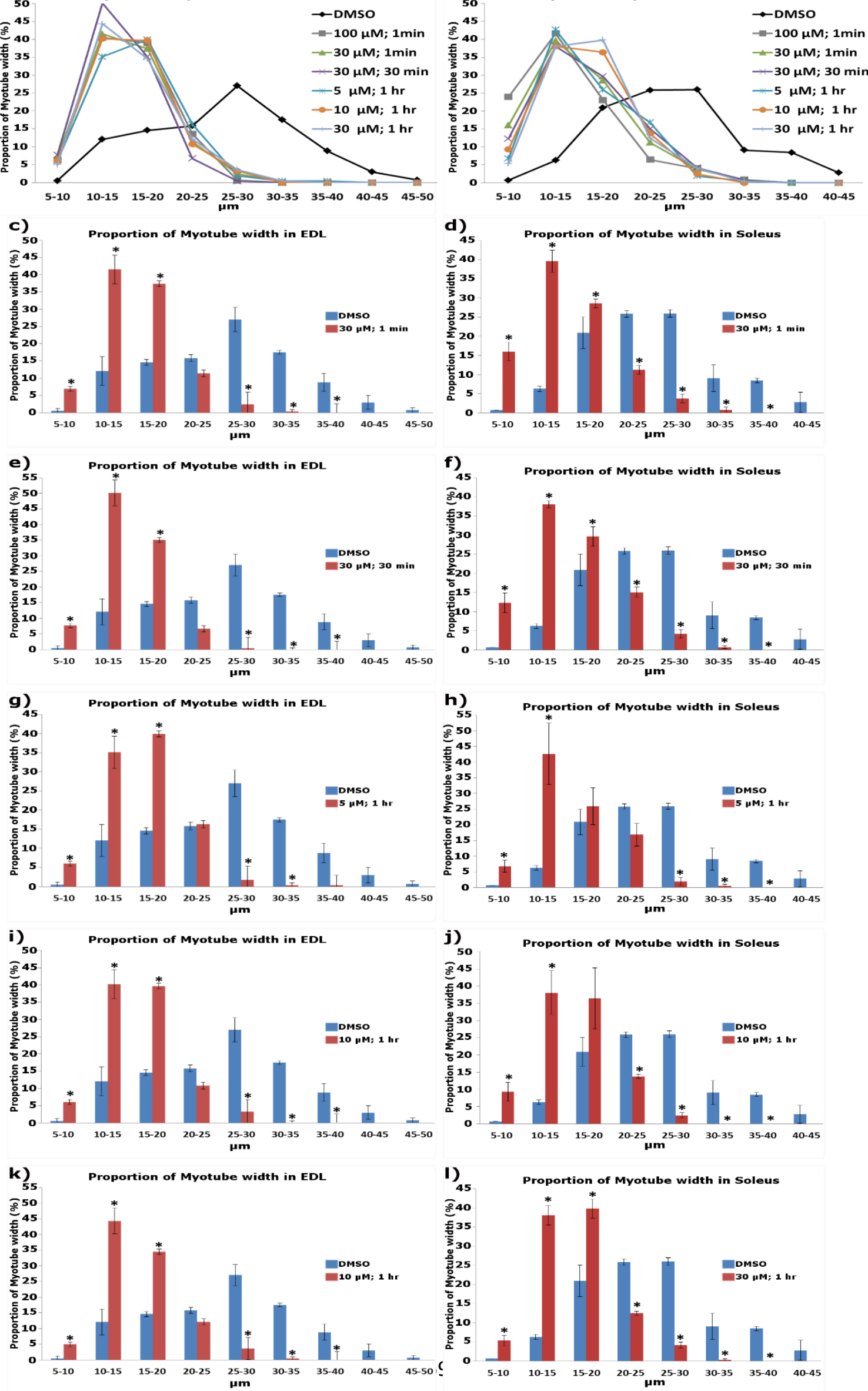
bioRxiv preprint doi: https://doi.org/10.1101/2020.09.27.315242; this version posted November 22, 2020. The copyright holder for this preprint (which was not certified by peer review) is the author/funder, who has granted bioRxiv a license to display the preprint in perpetuity. It is made available under aCC-BY-NC-ND 4.0 International license.

Ortuste Quiroga et al.

1361 Supplementary Figure 5. Yoda1-mediated activation of Piezo1 decreases myotube width.

1362 Myotube width was divided into incremental bins of $5 \mu \mathrm{m}$ and represented as percentages relative to the total number

1363 of myotubes counted. The overall proportion of myotube width distribution in DMSO controls and Yoda1-treated

1364 samples is summarised as line graphs in a) EDL and b) soleus-derived myotubes. c-l) Bar graphs comparing DMSO

1365 controls and samples which showed increased fusion post Yoda1 treatment in EDL (left) and soleus (right)-derived

1366 myotubes Data is mean \pm SEM from three experiments $(n=3$ mice $)$. *: Significant at $\mathrm{p}<0.05$ compared to DMSO

1367 controls conditions using one-way ANOVA followed by the Tukey-Kramer post-hoc. Please refer to Figure 6 a) and b)

1368 for representative images. 